Florida International University

FIU Digital Commons

6-26-2019

\title{
Implications of Non-equity Financing for Firm Financial Reporting Quality and Information Environment
}

Md Safayat Hossain

mhoss029@fiu.edu

Follow this and additional works at: https://digitalcommons.fiu.edu/etd

Part of the Accounting Commons, and the Corporate Finance Commons

\section{Recommended Citation}

Hossain, Md Safayat, "Implications of Non-equity Financing for Firm Financial Reporting Quality and Information Environment" (2019). FIU Electronic Theses and Dissertations. 4226.

https://digitalcommons.fiu.edu/etd/4226

This work is brought to you for free and open access by the University Graduate School at FIU Digital Commons. It has been accepted for inclusion in FIU Electronic Theses and Dissertations by an authorized administrator of FIU Digital Commons. For more information, please contact dcc@fiu.edu. 


\section{FLORIDA INTERNATIONAL UNIVERSITY}

Miami, Florida

\section{IMPLICATIONS OF NON-EQUITY FINANCING FOR FIRM}

FINANCIAL REPORTING QUALITY AND INFORMATION ENVIRONMENT

A dissertation submitted in partial fulfillment of the

requirements for the degree of

DOCTOR OF PHILOSOPHY

in

BUSINESS ADMINISTRATION

by

Md Safayat Hossain 
To: Dean Joanne Li

College of Business

This dissertation, written by Md Safayat Hossain, and entitled Implications of Non-equity Financing for Firm Financial Reporting Quality and Information Environment, having been approved in respect to style and intellectual content, is referred to you for judgment.

We have read this dissertation and recommend that it be approved.

\begin{tabular}{r} 
Kannan Raghunandan \\
\hline Clark M. Wheatley \\
\hline Abhijit Barua, Major Professor \\
is approved.
\end{tabular}

The dissertation of Md Safayat Hossain is approved.

$\begin{array}{r}\text { Dean Joanne Li } \\ \text { College of Business }\end{array}$
Andres G. Gil
and Dean of the University Graduate School

Florida International University, 2019 


\section{DEDICATION}

I would like to dedicate this thesis to my parents, Sarwar Jahan and Layla Begum. Without their blessings, support and love I, let alone this thesis, would be incomplete. I also dedicate this dissertation to my wife, Ummay Hani Tithy, whose love, support, and sacrifice in times of ease and in times of difficulties and restlessness enabled me to complete this journey. 


\section{ACKNOWLEDGMENTS}

I would like to thank Dr. Kannan Raghunandan, Dr. Clark Wheatley, and Dr. Qiang Kang, members of my dissertation committee for their continued support and invaluable suggestions. Especially, I would like to thank my adviser, Dr. Abhijit Barua for his guidance and motivation throughout the program. Without his motivation, endless care and exceptional guidance, this dissertation would not be possible. Finally, I would like to thank my fellow doctoral students in the accounting program at Florida International University for their unwavering help and suggestions. 


\title{
ABSTRACT OF THE DISSERTATION
}

\section{IMPLICATIONS OF NON-EQUITY FINANCING FOR FIRM}

\section{FINANCIAL REPORTING QUALITY AND INFORMATION ENVIRONMENT}

\author{
by \\ Md Safayat Hossain \\ Florida International University, 2019 \\ Miami, Florida \\ Professor Abhijit Barua, Major Professor
}

Prior studies in accounting and finance have extensively evaluated the way a firm interacts with, and subsequently responds to, its contracting agents under various conditions. Specifically, studies have explored the role of accounting information in mitigating agency conflicts and designing efficient contracts. My dissertation consists of three separate, albeit closely related, studies that extend the prior understanding of contracting relationships by documenting financial reporting outcomes of those relationships.

First, I investigate whether and how the presence of debt in the capital structure improves a firm's information environment. Empirical findings show that, after controlling for all known confounding factors, debt is negatively associated with information environment. Results using various measures of financial leverage indicate that analysts forecast accuracy (forecast dispersion) is high (low) for firms that use no/low debt. Additional analyses show that the effect of financial leverage is stronger for unrated firms, 
smaller firms, firms with low analysts following, and firm with low institutional shareholdings. Overall, results suggest that additional information generated by debt does not enhance information environment of a firm, rather firms with no/low leverage have incentives to provide superior information and facilitates market participants to make informed decisions. Second, I decompose leverage into operating and financing, and examine differential effects types of leverage on a firm's information environment. Results indicate that both operating liability leverage and financing leverage is negatively associated with the information environment of a firm. If I further decompose operating liability leverage into estimated and contractual, the negative association between leverage and information environment persists. Third, I investigate whether restrictive covenants imposed by lenders impact financial reporting quality of a firm. Primary empirical findings indicate that debt covenant strictness in general does not affect financial reporting quality of a firm. However, when separated out, strictness in capital covenant establishes less monitoring and thus reduces firm financial reporting quality. To address endogeneity, I use lender-specific financial shock as an instrumental variable (IV), suggested in Murfin (2012). The instrumental variable approach substantiates the findings. 
ESSAY 1: THE EFFECTS OF FINANCIAL LEVERAGE ON A FIRM'S INFORMATION ENVIRONMENT 1

I. INTRODUCTION 1

II. RELATED LITERATURE AND HYPOTHESIS DEVELOPMENT

III. METHOD 9

Proxies for Information Environment 9

Data Sources and Sample Construction $\quad 12$

$\begin{array}{ll}\text { IV. RESULTS } & 13\end{array}$

Descriptive Statistics 13

Regression Analyses $\quad 14$

V. ADDITIONAL ANALYSES 18

VI. CONCLUSION 20

REFERENCES $\quad 21$

$\begin{array}{ll}\text { APPENDIX A } & 47\end{array}$

ESSAY 2: THE EFFECTS OF OPERATING LIABILITY

LEVERAGE ON A FIRM'S INFORMATION ENVIRONMENT 48

I. INTRODUCTION 48

II. RELATED LITERATURE AND HYPOTHESIS DEVELOPMENT 51

III. METHOD 54

Proxies of Information Environment 54

Measures of Operating Liability Leverage 56

Sample and Data $\quad 56$

IV. RESULTS 57

Descriptive Statistics $\quad 57$

Regression Estimates $\quad 57$

V. ADDITIONAL ANALYSES 59

VI. CONCLUSION 61

REFERENCES $\quad 62$

$\begin{array}{ll}\text { APPENDIX B } & 75\end{array}$ 
ESSAY 3: DEBT COVENANT STRICTNESS AND FINANCIAL REPORTING QUALITY 77

I. INTRODUCTION 77

II. RELATED LITERATURE AND HYPOTHESIS
DEVELOPMENT

$\begin{array}{ll}\text { III. METHOD } & 87\end{array}$

Measures of Covenant Strictness $\quad 87$

Measures of Financial Reporting Quality (FRQ) $\quad 88$

Data and Sample $\quad 90$

IV. RESULTS 92

Descriptive Statistics $\quad 92$

Regression Estimates 93

V. CONCLUSION 95

REFERENCES $\quad 96$

$\begin{array}{ll}\text { APPENDIX C } & 108\end{array}$

$\begin{array}{ll}\text { VITA } & 109\end{array}$ 


\section{LIST OF TABLES}

TABLE

PAGE

ESSAY 1: THE EFFECTS OF FINANCIAL LEVERAGE LEVERGE ON A FIRM'S INFORMATION ENVIRONMENT

TABLE 1.1 Descriptive Statistics

25

26

TABLE 1.3 (a) Regression Analyses of Forecast Accuracy and

Forecast Dispersion on Financial Leverage

TABLE 1.3 (b) Regression Analyses of Firm-specific Information Environment on Financial Leverage

TABLE 1.4 (a) Regression Analyses of Forecast Accuracy and Forecast Dispersion on Leverage Decile

TABLE 1.4 (b) Regression Analyses of Firm-specific Information Environment on Leverage Decile

TABLE 1.5 (a) Regression Analyses of Forecast Accuracy and Forecast Dispersion on Leverage Tercile

TABLE 1.5 (b) Regression Analyses of Firm-specific Information Environment on Leverage Tercile

TABLE 1.6 (a) Regression Analyses of Forecast Accuracy and Forecast Dispersion on Zero Leverage

TABLE 1.6 (b) Regression Analyses of Firm-specific Information Environment on Zero Leverage

TABLE 1.7 (a) Regression Analyses of Forecast Accuracy and

Forecast Dispersion on Financial Leverage (Excluding Zero

Leverage)

TABLE 1.7 (b) Regression Analyses of Firm-specific Information Environment on Financial Leverage (Excluding Zero Leverage)

TABLE 1.8 (a) First-stage Regression Estimates to Calculate Predicted Leverage

TABLE 1.8 (b) Regression Analyses of Forecast Accuracy and Forecast Dispersion on Predicted Leverage

TABLE 1.8 (c) Regression Analyses of Firm-specific Information Environment on Predicted Leverage

TABLE 1.9 (a) Regression Analyses of Forecast Accuracy and Forecast Dispersion on Bank vs. Non-bank Debt 
TABLE 1.9 (b) Regression Analyses of Firm-specific Information Environment on Bank vs. Non-bank Debt

TABLE 1.10 Regression Analyses of Forecast Accuracy and Forecast Dispersion on Financial Leverage

ESSAY 2: THE EFFECTS OF OPERATING LIABILITY LEVERGE ON A FIRM'S INFORMATION ENVIRONMENT

TABLE 2.1 Descriptive Statistics 64

TABLE 2.2 Correlation Matrix 65

TABLE 2.3 (a) Regression Analyses of Forecast Accuracy and Forecast Dispersion on Leverage

TABLE 2.3 (b) Regression Analyses of Firm-specific Information Environment on Leverage

TABLE 2.4 (a) Regression Analyses of Forecast Accuracy and Forecast Dispersion on Estimated and Contractual Operating Liability Leverage

TABLE 2.4 (b) Regression Analyses of Firm-specific Information Environment on Estimated and Contractual Operating Liability Leverage

TABLE 2.5 Regression Analyses of Forecast Accuracy and Forecast Dispersion on Estimated and Contractual Operating Liability

ESSAY 3: DEBT COVENANT STRICTNESS AND FINANCIAL REPORTING QUALITY

TABLE 3.1 Descriptive Statistics

TABLE 3.2 Correlation Matrix

TABLE 3.3 Regression Analyses of Financial Reporting Quality on Debt Covenant Strictness

TABLE 3.4 Regression Analyses of Financial Reporting Quality on Debt Covenant Strictness

TABLE 3.5 Regression Analyses of Financial Reporting Quality on Debt Covenant Strictness (Using Instrumental Variable)

TABLE 3.6 Regression Analyses of Financial Reporting Quality on Debt Covenant Strictness (Using Instrumental Variable) 


\section{ESSAY 1: THE EFFECTS OF FINANCIAL LEVERGE ON A FIRM'S INFORMATION ENVIRONMENT}

\section{INTRODUCTION}

The objective of the study is to investigate whether the presence of debt in capital structure improves information environment of a firm. Capital structure theories suggest that debt generates additional information, works as a monitoring device, and hence improves overall information environment of a firm (Harris and Raviv 1990; Jensen 1986; Ross 1977). However, finance and accounting literature also indicate that debt accrues additional costs to the firm (Bolton and Scharfstein, 1990; Parsons and Titman, 2008), reduces financial flexibility (Harris and Raviv 1990; Zingales 1998), and increases agency conflicts (Jensen and Meckling 1976; Myers 1977), which may have negative effects on the information environment of the firm. Given the potential contrasting effects of debt financing on a firm's information environment, I examine the informational role of debt by investigating the association between level of debt and firm information environment.

Capital structure theories suggest that debt accrues benefits as well as costs to a firm's operating and financing environment ${ }^{1}$. An overreaching benefit of debt is that it generates information for market participants that helps to reduce information asymmetry. The mere ability of a firm to meet regular interest obligations sends information about firm; debtholders preempt negotiation, at event of default, also allows market participants to

\footnotetext{
${ }^{1}$ Potential benefits of debt include tax savings from interest paid to the debtholders (Kruas and Litzenberger 1973), reduced of agency costs due to restricted free cashflow (Jensen 1986), and enhanced monitoring by lenders and rating agencies. However, debt brings additional costs to the firm, such as distress or bankruptcy costs, enhanced agency conflicts between stockholder and bondholders (Jensen and Meckling,1976), increased financial misreporting etc.
} 
make informed decisions (Harris and Raviv 1990). Informational role of debt suggests that, when investors access to firm-specific information is limited, debt establishes additional control and generate information that would other be inaccessible or costly. Moreover, debt signaling theory posits that managers of high-quality firms send out signals about quality of the firm through debt issuance (Ross 1977). Furthermore, by generating additional information, debt reduces uncertainty associated with future cash flows, which decreases default probability (Merton 1974). Furthermore, by limiting managerial discretion over free cash flows, managers are restraints from their 'empire building' intent (Jensen 1986). In addition, debt disciplines managers by making sure that respective debt covenants are maintained (Jensen and Meckling 1976) and debt obligations are serviced (Harris and Raviv 1990). Studies also show that lenders require reliable and timely information to make lending decisions; similarly, rating agencies demand access to timely information to make rating recommendations. As a consequence, debt facilitates market participants to get access to firm-specific information that reduce information asymmetry and private information search incentives.

However, with the use of debt risk of financial distress (Parsons and Titman 2008), and default probability (Harris and Raviv 1991) increase, whereas financial flexibility (Zingales 1998), probability of reorganization after default (Harris and Raviv 1990) decrease. Moreover, debt can enhance agency conflicts between debtholders and shareholders, which can have adverse effects on firms' information environment. In addition, managers' propensity to misstate financial statement increases as debt increases. Debt covenant hypothesis argues that if a firm is highly levered managers more likely to manipulate the financial statements to avoid covenant violation (Watts and Zimmerman 
1986). Studies examining zero leverage firms report that firms with low/no debt are more profitable, pay higher dividends, higher taxes, and have equally effective internal and external governance mechanisms in place (Devos et al., 2012; Strebulaev and Yang 2013). Managers of these firms enjoy the benefit of financial flexibility. Even in absence of debt, managers of these firms may choose to generate superior information to the market so that they can obtain future financing at a lower cost. Given the competing predictions surrounding the role of debt, it is an open empirical question that how debt in the capital structure effects firm's information environment.

To proxy for information environment quality, I use two analysts forecast-based measures - analysts' forecast accuracy and forecast dispersion, and three firm-specific measures-idiosyncratic volatility, synchronicity, and PIN. I use 23,584 firm-year observations for a sample period between 1989 and 2017. To empirically test the informational role of debt, I regress empirical proxies for information environment on several continuous as well as discrete measures of financial leverage. In general, results refute informational role of debt and indicate that, after controlling for all known confounding factors, debt is negatively associated with information environment. Results from various measures of financial leverage show that forecast accuracy (forecast dispersion) is high (low) for firms that use no or low debt. Additional analyses reveal that the effect of financial leverage is more pronounced for unrated firms, smaller firms, firms with low analysts following, and low institutional shareholdings. Overall, results indicate that additional information generated by debt does not contribute to the information environment of a firm, rather firms with no or low leverage have incentives to provide superior information that facilitates market participants to make informed decisions. 
Reminder of the paper develops as follows. Section II outlines related literature and develops testable hypotheses. Section III discusses variable measurements and the research design. Section IV presents baseline results and section $\mathrm{V}$ discusses results from additional tests. Section VI concludes the study.

\section{RELATED LITERATURE AND HYPOTHESIS DEVELOPMENT}

Harris and Raviv (1990) document that debt generates additional information about a firm's operating environment in two distinct scenarios. First, the mere ability of a firm to meet regular interest obligations sends information about its competence to generate continued stream of cash flows. Second, at the event of default, debtholders preempt negotiation, as well as their capability to force a firm into liquidation, allows market participants to make informed decisions, including changes in operating policy and reorganization of the financial structure. These aspects of debt suggest that, when investors' access to firm-specific information is limited, they prefer lenders' monitoring and additional information generated through debt in the capital structure (Jensen 1986). Therefore, with the change in level of debt, information available about the firm in the market is expected to change. Moreover, information asymmetry theories indicate that firm-specific information is not symmetrically distributed between firm insiders and outsiders. To mitigate information asymmetry, managers of high-quality firms send out

signals about quality of the firm through debt issuance (Ross 1977). Therefore, debt issuance, too, carries additional information for the market participants.

Furthermore, debt can reduce default risk by producing additional information that reduces default probability by eliminating cash flows related uncertainty (Merton 1974). 
Additional information obtained through debt also decreases information risk by reducing information asymmetry (Easley and O'Hara 2004), which again lowers default risk (Mansi et al. 2006).

In addition to its informational role, debt works as a disciplining device. Jensen and Meckling (1976) posit that debt reduces agency conflict arising from the separation of ownership and control. Agency theories suggest that some managers use debt conservatively from their empire-building desires; they optimize their personal utility functions and pursue private objectives by controlling corporate resources (Morellec et al. 2012). Debt, in this respect, limits managerial discretion over free cash flows and puts constraints over managers' 'empire building' intent (Jensen 1986). It also disciplines managers by monitoring managerial actions to make sure that respective debt covenants are maintained (Jensen and Meckling 1976) and debt obligations are serviced (Harris and Raviv 1990). In addition, with the issuance of debt, managers work hard, reduce wasting free cash flows, and make better investment decisions (Grossman and Hart 1982). In addition to overseeing managerial actions, debt establishes control over financial reporting system. To make new lending decisions and to evaluate existing ones, lenders require reliable and timely information. Similarly, to make rating recommendations, debt analysts, e.g., rating agencies, demand timely information. Therefore, debt facilitates market participants to gain access to information that would otherwise be unidentified.

\section{Information cost of debt:}

As opposed to generating additional information for market participants, debt accrues costs to the operating environment of a firm. Finance theories argue that as debt 
increases distress risk (Parsons and Titman 2008) and default probability (Harris and Raviv 1991) increase, however, financial flexibility (Zingales 1998) and probability of postdefault reorganization (Harris and Raviv 1990) decrease. Excessive use of debt also affects operating performance by worsening the competitive position of a firm and disrupting its relationship with suppliers, key employees and customers (Bolton and Scharfstein 1990; Parsons and Titman 2008).

In addition, agency theories suggest that a multitude of agency conflicts may arise from the issuance of debt (Armstrong et al. 2010). First, equity holders and debtholders may fight over dividends: equity holders limit resources available to pay off debt and thereby increase their dividend income. Second, existing debtholders may consider potential debt issue a threat to regular payment of existing debts. Third and fourth source of conflicts relate to asset substitution (Jensen and Meckling 1976) and under-investment problem (Myers 1977). Jensen and Meckling (1976) argue that, in the presence of debt, shareholders would undertake riskier projects and enjoy benefits of future cash flows, taking less risk of downside losses. By substituting less risky projects by riskier ones, shareholders can increase their wealth at the cost of debtholders. Myers (1977), on the other hand, posit that shareholders receive residual cash flows after servicing debt and thus have incentives to decline projects that just pay debtholders and leave nothing for shareholders. Firms, as a result, forgo projects with positive NPV and remain under-invested.

Another overreaching cost of debt is that it provides incentive for management to misstate financial information. Debt covenant hypothesis argues that managers of highly levered firms try to avoid covenant violation by manipulating financial statements (Watts 
and Zimmerman, 1986). Consistent with this argument, prior studies report a positive association between leverage and income increasing accounting choices (e.g., Bowen et al., 1995; Zmijewski and Hagerman 1981), and leverage and real earnings management in the form of sale of assets (Bartov 1993).

\section{Why do firms use less leverage?}

Even though the benefits of using debt is more pronounced in the literature, many firms use less debt than that is optimally desirable (Graham 2006). Studies find that low/no debt firms are highly profitable: profitable firms accumulate larger retained earnings, pay off debt and, as a result, become less levered (Lemmon et al 2008; Frank and Goyel 2007). Donaldson and Preston (1995) observe that managers prefer to remain under-levered by financing new investment with retained earnings rather than debt. Strebulaev and Yang (2013) report that, compared with control firms, firms with low/no debt are more profitable, pay higher taxes, higher dividends, and hold greater cash balances. From a similar sample, Devos et al. (2012) conclude that the choice of zero or low leverage is not driven by entrenched managers attempting to avoid monitoring by lenders. They find that firms with low leverage establish equally effective governance mechanisms, and initiation of new debt does not come with additional monitoring pressure or takeover threats. In contrast, studies show that firms using excess leverage experience losses, are in financial distress and in economic hardship (Korteweg 2010; Caskey et al 2012). As a consequence, such firms are less likely to benefit from tax shields and reduced agency costs and are more likely to suffer from the potential costs of financial distress. 


\section{Benefit of improved information environment for future financing:}

Finance theories dictate that firms' potential source of financing is determined by the extent of mispricing of securities in the market. Lesser known firms are at greater risk of mispricing and hence finance internally. Biddle and Hillary (2006) note that lack of adequate information force investors to withhold capital; higher quality accounting information in such cases reduces information asymmetry and facilitates external financing. Studies also find that firms requiring external financing reduce information asymmetry by issuing earnings forecasts more frequently than others (Ruland et al., 1990; Frankel et al., 1995), and supply financial information in a fashion that reduces cost of such financing. Bharat et al. (2008) document that firms with poor accounting quality choose private debt over public debt, post more collateral and are charged higher interest rates. Similarly, Chava et al. (2010) find that firms with poor accounting quality face dividend payout restrictions. These results suggest that managers of firms with low, or no debt, have incentives to supply higher quality financial information and reduce information asymmetry that would facilitate the firm obtain financing at lower cost.

Although the informational role of debt is well established in prior literature, the association between firms use of debt and information environment is less straightforward. From the above discussion, I contend that debt improves information environment by (1) generating additional information about firm's operation, (2) establishing better monitoring over managerial actions, (3) limiting managerial discretion over cash flow and hence reducing agency conflict, and (4) improving financial reporting quality. In contrast, debt can worsen information environment by increasing (1) liquidity and bankruptcy risk, (2) 
managements' intent to misreport financial information, and (3) agency conflicts between bondholder and shareholder. In addition, with no/low debt, managers may choose to generate superior information to the market so that information asymmetry is reduced, and future financing can be obtained at a lower cost. Given the tension surrounding the role of debt in information environment, I hypothesize that (stated in null form):

$\mathrm{H}_{0}$ : Ceteris paribus, debt in the capital structure does not affect information environment of a firm.

\section{METHOD}

\section{Proxies for Information Environment:}

I primarily use two analysts forecast-based measures of information environment forecast accuracy (ACCURACY) and forecast dispersion (DISPERSION). Prior studies illustrate that equity analysts supply additional information to the market participants that reduces information asymmetry and improves a firm's information environment (Lang, Lins and Miller 2004; Mansi, Maxwell and Miller 2011). Analysts collect and process unique set of data and work as equity specialists (Piostroski and Roulstone 2004). Studies document that analysts' forecasts and recommendations are value relevant and thus affect stock prices (Givoly and Lakonishok 1979) as well as rating recommendations (Cheng and Subramanyam 2008). Additionally, studies argue that analysts' information production works as an alternative mentoring mechanism: it establishes market discipline from their earnings forecasts based-information (DeFond and Hung 2007). Therefore, with superior information in place, uncertainty surrounding firms' operating environment is reduced and analysts' ability to forecast earnings is improved. From prior studies, I thus draw that better 
information environment is associated with higher analysts' forecast precision and lower forecast dispersion. To examine the relation between debt and information environment, I use the following model:

$$
\begin{aligned}
& \text { ACCURACY } Y_{i, t} \text { or DISPERSION } \text { DI,t }=\beta_{0}+\beta_{1} \text { FINLEV }_{i, t}+\sum_{k} \beta_{k} \text { FirmControls }+ \\
& \text { IndustryFE }+ \text { YearFE }+\varepsilon
\end{aligned}
$$

To proxy for information environment, I define forecast accuracy as the negative of absolute value difference between the actual EPS and the median analysts forecast scaled by year-end stock price. I define forecast dispersion as the standard deviation of analysts' forecasts (forecast dispersion) scaled by ending stock price. To study the effect of financial leverage, I include continuous as well as discrete measures of debt. To control for firm fundamentals that potentially could drive both leverage and analysts' forecast, I include (1) $\log$ of market capitalization at the beginning of the year (SIZE), (2) market to book ratio $(M B),(3)$ changes in earnings per share over year $t\left(C h \_E P S\right),(4)$ an indicator variable for negative profit (LOSS), and (5) standard deviation of return-on-assets over last five years $\left(S T D \_R O A\right)$. I also control for log of number of analysts ( $\left.N \_A N A L Y S T\right)$ and log of number of days between analysts forecast and earnings announcement (HORIZON). In an extended model, I further control for firm operating liability leverage (OPLLEV), asset tangibility (TANGIBILITY), audit quality (BIG4), dividend paying status (DIVPAYER), volatility of cash flow (STD_CFO), and total accruals (ACCRUALS).

\section{Firm-specific Measures of Information Environment}

The level and type of information advantage across different market participants are not the same. Market participants have asymmetric access to firm-, industry-, and 
market-specific information. Piotroski and Roulstone (2004) note that market participants' influence over information environment is a function of individual participants' relative information advantage. In the lending process, lenders obtain more precise, firm-specific information at relatively lower costs. Information access by the lender is more likely to impact the information environment of a firm via firm-specific component, then via industry-, or market-wide components. To capture firm-specific information environment I use three measures - idiosyncratic volatility, synchronicity, and probability of informed trading.

Following Brockman and Yan (2009), my constructs for idiosyncratic volatility and synchronicity are derived from regressing weekly stock returns on contemporaneous and lagged weekly market returns, as well as on contemporaneous and lagged weekly industry returns. Idiosyncratic volatility is calculated as the standard deviation of regression residuals, whereas synchronicity is estimated from log transformation of unbounded $\mathrm{R}^{2} .^{2}$

$$
R E T_{i, t}=\beta_{0}+\beta_{1} M_{K T R E T}+\beta_{2} M_{K T R E T} T_{t-1}+\beta_{3} I N D R E T_{t}+\beta_{4} I N D R E T_{t-1}+\varepsilon_{i, t}
$$

The probability of informed trading $(P I N)$ is estimated from a trading model where trade order follows a combined Poisson process (Easley et al., 2002). However, I use extended PIN measure suggested by Brown and Hillegeist $(2007)^{3}$. I calculate all three

\footnotetext{
${ }^{2}$ Following Morck et al. (2000), I calculate synchronicity as $S Y N C H_{i, t}=\log \left(\frac{R^{2}}{1-R^{2}}\right)$.

${ }^{3}$ I use annual PIN data for period covering 1993 to 2010 available at Professor Stephen Brown's website (http://scholar.rhsmith.umd.edu/sbrown/pin-data).
} 
firm-specific measures, idiosyncratic volatility (IDVOL), synchronicity $(S Y N C H)$ and PIN for each firm-year and estimate the following model:

$$
\begin{gathered}
\text { IDVOL }_{i, t} \text { or SYNCH } \\
\text { IndustryFE }+ \text { or PIN } \\
\text { YearFE }+\varepsilon
\end{gathered}
$$

To isolate the effects of debt on firm-specific information environment, I control for confounding factors suggested in previous studies (Piotroski and Roulstone, 2004; Brockman and Yan 2010). Specifically, I control for firm size (SIZE), average daily turnover $(T R V O L)$, standard deviation of return-on-assets $\left(S T D V \_R O A\right)$, operating leverage (OPLLEV), analysts following ( $\left.N \_A N A L Y S T\right)$, and firm visibility (SP500). In an extended model, I further control for Herfindahl industry concentration index (HERFIN), number of firms in the industry group (NFIRM), and firm-industry earnings correlation (ROACORR).

\section{Data Sources and Sample Construction}

Data for this study come from several sources: accounting data are obtained from COMPUSTAT fundamental annual database, price and return data come from CRSP database, institutional ownership data are derived from Thompson Reuters Institutional Holdings database, and analyst forecast data originate from I/B/E/S database. Initial sample includes firm-year observations between 1989 and 2017. Following prior studies, I exclude firms incorporated outside USA and firms in the financial (SIC 6000-6999) and utility (SIC 4900-4999) sector. To avoid potential data error and outliers, I exclude observations with 
less than one million in market capitalization, market price less than a-dollar ${ }^{4}$ and observations outside zero and unit leverage (Flannery and Rangan 2006; Warr et al. 2012). To ensure sufficient observations for the estimation of analysts' forecast accuracy and dispersion, I require firms to be followed by at least three analysts. After deleting observations with missing values, continuous variables are winsorized at top and bottom one percent. Final sample consists of 23,584 firm-year observations for 4,419 unique firms.

\section{RESULTS}

\section{Descriptive Statistics}

Table 1.1 presents summary statistics. On an average, firms finance $22 \%$ of their total assets by financial debt (FINLEV), like bond, and bank loans, while 28\% (OPLLEV) of their total assets are financed by non-financial liabilities, like accounts payable, taxes payable and accrued expenses. However, during the sample period $12 \%$ firms had no financial debt in their capital structure $(Z L)$, which is consistent with the zero-leverage puzzle (Strebulaev and Yang 2013). Since I restrict the sample to the firms covered by at least three analysts, sample firms relatively old, large and profitable. The mean (median) size of firms is $\$ 7,269(\$ 1,310)$ million and the mean (median) market-to-book ratio is 3.53 (2.42). 65 percent shares are owned by institutional investors, 74 percent firms are audited by Big4 audit firms, and 48 percent firms pay dividend. These statistics are comparable with statistics reported in recent studies (Brockman and Yan 2009).

\footnotetext{
${ }^{4}$ Ratios scaled by market capitalization or price blow up if the denominator is smaller than one. Frank and Goyel (2007) also observe that data problems are much more common for smaller firms. Restricting sample to relatively large firms could avoid the data problems.
} 
Table 1.2 reports Pearson pairwise correlation coefficients. Correlation coefficients indicate that financial leverage is negatively (positively) correlated with analysts' forecast accuracy and forecast dispersion. Among the components of total leverage, financial leverage (FINLEV) and operating liability leverage (OPLLEV) are negatively (-0.2650) correlated. The relation between bank debt and non-bank debt is also negative (-0.2027). These associations suggest that firms substitute one type of debt with another. Table 1.2 also indicate that the correlation coefficients are not significantly high enough to create multi-collinearity problem in regression estimations. ${ }^{5}$

\section{Regression Analyses - Forecast Accuracy and Dispersion:}

Table 1.3(a) presents results of primary analyses. Column 1 through 4 report regression results for forecast accuracy, and column 5 through 8 report the same for forecast dispersion. I primarily use the baseline regression for initial results, and then I extend the model including additional control variables that are potentially associated with the dependent variable as well as the variable of interests, leverage. After controlling all known determinates, results indicate that the level of financial leverage is negatively (pvalue <.001) associated with forecast accuracy and positively associated with (p-value $<.001)$ forecast dispersion. In contrast with informational role of debt, I find that firms with lower financial leverage has better information environment. As expected, I also find forecast accuracy (dispersion) to be positively (negatively) associated with size, market to book ratio and institutional holdings, and negatively (possibly) associated with forecast

\footnotetext{
${ }^{5}$ Variance inflation factors (VIF) in all regression specifications are less than 3.
} 
horizon, earnings persistence and analysts following. I document similar results in an extended model.

\section{Regression Analyses - Firm-specific Information Environment:}

I report regression estimates of firm-specific information environment of financial leverage in Table 1.3(b). Results for the idiosyncratic volatility (IDVOL) are in the first four columns, results for synchronicity $(S Y N C H)$ in the middle four columns and results for probability of informed trading (PIN) are in the final four columns. The base model tests primary association between the dependent variable and the variable of interest, whereas an extended model is used to control for proxies for additional industry attributes. Regression results for idiosyncratic volatility (IDVOL) show that the coefficient for financial leverage is (FINLEV) is negative and statistically significant. After controlling for industry-specific attributes, results remain the same. These results are consistent with the previous findings that firm-specific information is low for high leverage firms and vice versa. Coefficient estimates for synchronicity $(S Y N C H)$ and is negative but are not statistically significant. Regression estimates for PIN are positive but statistically not significant as well.

\section{Regression Analyses - Alternative Measures of Leverage:}

To confirm the findings reported in Table 1.3(a) and 3(b), I next use a few discrete measures of financial leverage. First, instead of using level of financial leverage (FINLEV), I use the annual decile rank of financial leverage $\left(L E V_{-} D E C\right)$ as the variable of interest. Table 1.4(a) and (b) respectively repeats the analysis with leverage decile and reports results consistent with those reported in Table 1.3(a) and 1.3(b). Second, I use an indicator 
variable for the tercile ranked observations based on financial leverage. Top tercile firmyear observations are compared against the bottom tercile observations with an indicator variable $(L O W)$. Results in the Table 1.5(a) indicate that the analysts' forecast accuracy (dispersion) for the bottom tercile firms are higher (lower) than that for the top tercile firms. These findings substantiate the results reported in baseline regression. As reported earlier, $12 \%$ firms in the sample do not use any financial leverage in their capital structure. Thus, there is a possibility that the results reported in above analyses are biased towards levered firms. I address this possibility in two ways: I re-run the regressions using an indicator variable for zero leverage firms $(Z L)$, and a separate regression excluding all zero levered firms. After controlling for all other known factors, I find consistent result in table 1.6(a) and table 1.7(a) - forecast accuracy (dispersion) is higher (lower) in the zero levered or low levered firms than highly levered firms. All the models in my analyses have significant explanatory power, explaining about 35\% (25\%) of the cross-sectional variations of analysts forecast accuracy (dispersion). While results across analysts-forecast based are consistent, results for firm-specific information environment do not consistently refute the informational role of debt. Coefficient estimates for IDVOL has remained statistically significant across all measures of leverage and support results from analysts forecast based measures. However, coefficient estimates for SYNCH and PIN are not always statistically significant.

\section{Two-stage Regression Analyses:}

While results in table 1.3 through table 1.7 refute the informational role of debt, I next address endogeneity concern by using a two-stage regression estimation. In the first 
stage, I estimate expected level of financial leverage by regressing actual debt ratios on firm specific characteristics suggested in prior studies. I then use the predicted leverage ratio as a proxy of financial leverage in the baseline regression. The calculation of estimated leverage relies on Fama and French (2002) and uses the Fama and MacBeth (1973) annual cross-sectional regressions estimates. The following equation estimates the predicted value of financial leverage:

$$
T \widehat{L E V}_{l, t}=\hat{\beta} X_{i, t-1}
$$

Consistent with the literature, we select the following firm specific characteristics $\left(X_{i, t}\right)$ as determinants of a firm's target leverage (Frank and Goyal 2007; Flannery and Rangan 2006): firm size (natural logarithm of total assets adjusted for inflation), profitability (operating income scaled by assets), market-to-book ratio, depreciation (scaled by assets), asset tangibility (net PPE scaled by assets), R\&D expenses (scaled by sales), an indicator variable for reporting $\mathrm{R} \& \mathrm{D}$ expenses, and industry median leverage (calculated for each year based on Fama and French 48 industry classification). All continuous variables used in this estimation are winsorized at top and bottom 1 percent. Table 1.8(a) presents first-stage regression estimates. As expected, firm size, growth, tangibility and industry median leverage ratio are positively associated with a firm's leverage, while profitability, depreciation and research and development expenses are negatively associated. I report the findings from second-stage regression for forecast accuracy and forecast dispersion in table 1.8(b), and that for idiosyncratic volatility, synchronicity and PIN in table 1.8(c). Regression estimates confirms the prior findings that financial leverage is negatively (positively) associated with analysts' forecast accuracy (dispersion). Taken together, results reject the informational role of debt. Regression 
results indicate that firms with no/low debt maintains better information environment than their levered counterparts.

\section{ADDITIONAL ANALYSES}

In the main analyses, I document that financial leverage weakens a firm's information environment. Financial leverage is negatively associated with analysts' forecast accuracy and positively associated with forecast dispersion. However, studies also argue that a firm's information environment is also a function of other firm fundamentals, such as size, rating status, analysts following and institutional shareholdings. To test whether the relation between financial leverage and firm information environment are conditional on such factors, I split the sample based on these variables and rerun the regression model separately. Results are reported in Table 1.10. For brevity, I only report the regression estimates of the variable of interest financial leverage (FINLEV).

\section{Rated versus Unrated Firms}

A firm's information environment is shaped by several information intermediaries, credit rating agencies being the influential ones. If a firm is rated, it is highly likely that the firm's information environment is be superior than the firm which is unrated. To test the differential effect of rating, I rerun regressions reported in the main analyses separately in two sub-samples: rated firms and unrated firms. Results are reported in the first two

columns of Panel A of Table 1.10. In both-sub samples the coefficients of financial leverage $($ FINLEV) are negative (positive) and significant $(\mathrm{p}<.001)$ for analysts' forecast accuracy (dispersion). However, the coefficient estimates for unrated firms are stronger than those for rated firms. This indicates that, absent information from rating agencies, the effect of debt is more pronounced on information environment. 


\section{Low versus High Analysts Following}

Firms that are followed by fewer analysts are more likely to have weaker information environment. To test if analysts following affects the association between financial leverage and information environment, I divide the sample based on median value of number of analysts into two sub-samples: low analysts following, and high analysts following and rerun the bassline regression. Results are reported in the first two columns of Panel B of Table 1.10. Consistent with previously documented results, the coefficient of FINLEV is significantly $(\mathrm{p}<.001)$ negative (positive) for analysts forecast accuracy (dispersion). However, results indicate that when analysts monitoring is low, the role of leverage becomes stronger.

\section{Low versus High Institutional Shareholdings}

Theories suggest that institutional shareholdings generate additional information for market participants and that managers pay more attention to avoid disciplinary actions from institutional shareholders. To investigate whether monitoring from institutional shareholdings affect the relation between leverage and information environment, I estimate the baseline model on two subsamples: low institutional firms and high institutional firms. Results are reported in Panel $\mathrm{C}$ of Table 1.10. Consistent with prior findings, FINLEV is significantly ( $\mathrm{p}<.001)$ negatively (positively) associated with analysts forecast precision (dispersion). However, the effect is more pronounced when monitoring from external shareholders is low.

\section{Large versus Small Firms}

To investigate the effects of size differentials, I conduct separate regressions for sub-samples - large firms and small firms, where large (small) firms are those with total 
assets higher (lower) than the annual median. Panel D of Table 1.10 shows that the coefficient of FINLEV is negative and significant $(\mathrm{p}<.01)$ for forecast accuracy, and positive and significant $(\mathrm{p}<.001)$ for forecast dispersion. However, the effects are higher for smaller firms than for larger firms. A potential explanation for the stronger effects of in smaller firms is that these firms are characterized by weaker corporate governance, internal control systems and external monitoring. Studies also suggest that smaller firms are riskier than larger firms (Beaver, Kettler and Scholes 1970; Fama and French 1993). Thus, any change in information environment with respect to change in financial leverage is more pronounced for smaller firms than for larger firms.

\section{CONCLUSION}

Countervailing arguments surrounding debt predicts that debt could either improve or weaken information environment of a firm. On set of theories argue that debt provides additional information for market participants and hence improves overall information set of a firm (e.g., Harris and Raviv 1990; Jensen 1986; Ross 1977). On the other hand, an alternative set of theories posit that debt accrues additional costs, reduces financial flexibility, and increases agency conflicts, thereby decreasing information quality of the firm. Given the potential contrasting effects of debt financing on a firm's information environment, in this study I examine the informational role of debt by investigating the association between level of debt and firm information environment.

Using analysts' forecast accuracy and forecast dispersion as analysts forecast-based measures, and idiosyncratic volatility, synchronicity, and PIN as firm-specific measure, I find that report results against the informational role of debt. Findings of this study suggest 
that debt is negative associated with information environment, primarily driven by additional agency cost of debt, reduced financial flexibility and increased misreporting risk. Results further show that the effect of financial leverage is stronger for unrated firms, smaller firms, firms with low analysts following, and firm with low institutional shareholdings. Overall, findings of this study contribute to the accounting and finance literature that relate spillover effects of lender monitoring.

\section{REFERENCES}

Armstrong, C.S., Guay, W.R. and Weber, J.P., 2010. The role of information and financial reporting in corporate governance and debt contracting. Journal of Accounting and Economics, 50(2), pp.179-234.

Bartov, E., 1993. The timing of asset sales and earnings manipulation. Accounting Review, pp.840-855.

Beaver, W., Kettler, P. and Scholes, M., 1970. The association between market determined and accounting determined risk measures. The Accounting Review, 45(4), pp.654682.

Bharath, S.T., Sunder, J. and Sunder, S.V., 2008. Accounting quality and debt contracting. The Accounting Review, 83(1), pp.1-28.

Biddle, G.C. and Hilary, G., 2006. Accounting quality and firm-level capital investment. The Accounting Review, 81(5), pp.963-982.

Bolton, P. and Scharfstein, D.S., 1990. A theory of predation based on agency problems in financial contracting. The American economic review, pp.93-106.

Bowen, R.M., DuCharme, L. and Shores, D., 1995. Stakeholders' implicit claims and accounting method choice. Journal of accounting and economics, 20(3), pp.255295.

Brockman, P. and Yan, X.S., 2009. Block ownership and firm-specific information. Journal of Banking \& Finance, 33(2), pp.308-316.

Brown, S. and Hillegeist, S.A., 2007. How disclosure quality affects the level of information asymmetry. Review of Accounting Studies, 12(2-3), pp.443-477. 
Caskey, J., Hughes, J. and Liu, J., 2012. Leverage, excess leverage, and future returns. Review of Accounting Studies, 17(2), pp.443-471.

Chava, S. and Purnanandam, A., 2010. CEOs versus CFOs: Incentives and corporate policies. Journal of Financial Economics, 97(2), pp.263-278.

Cheng, M. and Subramanyam, K.R., 2008. Analyst following and credit ratings. Contemporary Accounting Research, 25(4), pp.1007-1044.

DeFond, M.L. and Hung, M., 2007. Investor protection and analysts' cash flow forecasts around the world. Review of Accounting Studies, 12(2-3), pp.377-419.

Devos, E., Dhillon, U., Jagannathan, M. and Krishnamurthy, S., 2012. Why are firms unlevered?. Journal of corporate finance, 18(3), pp.664-682.

Donaldson, T. and Preston, L.E., 1995. The stakeholder theory of the corporation: Concepts, evidence, and implications. Academy of management Review, 20(1), pp.65-91.

Easley, D. and O'hara, M., 2004. Information and the cost of capital. The journal of finance, 59(4), pp.1553-1583.

Fama, E.F. and French, K.R., 1993. Common risk factors in the returns on stocks and bonds. Journal of financial economics, 33(1), pp.3-56.

Fama, E.F. and French, K.R., 2002. Testing trade-off and pecking order predictions about dividends and debt. The review of financial studies, 15(1), pp.1-33.

Fama, E.F. and MacBeth, J.D., 1973. Risk, return, and equilibrium: Empirical tests. Journal of political economy, 81(3), pp.607-636.

Flannery, M.J. and Rangan, K.P., 2006. Partial adjustment toward target capital structures. Journal of financial economics, 79(3), pp.469-506.

Frank, M.Z. and Goyal, V.K., 2007. Trade-off and pecking order theories of debt.

Frankel, R., McNichols, M. and Wilson, G.P., 1995. Discretionary disclosure and external financing. Accounting Review, pp.135-150.

Givoly, D. and Lakonishok, J., 1979. The information content of financial analysts' forecasts of earnings: Some evidence on semi-strong inefficiency. Journal of Accounting and Economics, 1(3), pp.165-185.

Graham, J.R., 2006. A review of taxes and corporate finance. Foundations and trends ${ }^{\circledR}$ in finance, 1(7), pp.573-691. 
Grossman, S.J. and Hart, O.D., 1982. Corporate financial structure and managerial incentives. In The economics of information and uncertainty (pp. 107-140). University of Chicago Press.

Harris, M. and Raviv, A., 1990. Capital structure and the informational role of debt. The Journal of Finance, 45(2), pp.321-349.

Harris, M. and Raviv, A., 1991. The theory of capital structure. the Journal of Finance, 46(1), pp.297-355.

Jensen, M.C. and Meckling, W.H., 1976. Theory of the firm: Managerial behavior, agency costs and ownership structure. Journal of financial economics, 3(4), pp.305-360.

Jensen, M.C., 1986. Agency costs of free cash flow, corporate finance, and takeovers. The American economic review, 76(2), pp.323-329.

Korteweg, A., 2010. The net benefits to leverage. The Journal of Finance, 65(6), pp.21372170 .

Kraus, A. and Litzenberger, R.H., 1973. A state-preference model of optimal financial leverage. The journal of finance, 28(4), pp.911-922.

Lang, M.H., Lins, K.V. and Miller, D.P., 2004. Concentrated control, analyst following, and valuation: Do analysts matter most when investors are protected least? Journal of Accounting Research, 42(3), pp.589-623.

Lemmon, M.L., Roberts, M.R. and Zender, J.F., 2008. Back to the beginning: persistence and the cross-section of corporate capital structure. The Journal of Finance, 63(4), pp.1575-1608.

Mansi, S.A., Maxwell, W.F. and Miller, D.P., 2011. Analyst forecast characteristics and the cost of debt. Review of Accounting Studies, 16(1), pp.116-142.

Merton, R.C., 1974. On the pricing of corporate debt: The risk structure of interest rates. The Journal of finance, 29(2), pp.449-470.

Morellec, E., Nikolov, B. and Schürhoff, N., 2012. Corporate governance and capital structure dynamics. The Journal of Finance, 67(3), pp.803-848.

Myers, S.C., 1977. Determinants of corporate borrowing. Journal of financial economics, 5(2), pp.147-175.

Parsons, C. and Titman, S., 2008. Handbook of Corporate Finance: Empirical Corporate Finance. Capital structure and corporate strategy. 
Piostroski, J. and Roulstone, D., 2004. The influence of analysts, institutional investors, and insiders on the incorporation of market, industry, and firm-specific information to stock prices. Accounting Review, 79, pp.1119-1151.

Ross, S.A., 1977. The determination of financial structure: the incentive-signalling approach. The bell journal of economics, pp.23-40.

Ruland, W., Tung, S. and George, N.E., 1990. Factors associated with the disclosure of managers' forecasts. Accounting Review, pp.710-721.

Strebulaev, I.A. and Yang, B., 2013. The mystery of zero-leverage firms. Journal of Financial Economics, 109(1), pp.1-23.

Warr, R.S., Elliott, W.B., Koëter-Kant, J. and Öztekin, Ö., 2012. Equity mispricing and leverage adjustment costs. Journal of Financial and Quantitative Analysis, 47(3), pp.589-616.

Watts, R.L. and Zimmerman, J.L., 1986. Positive accounting theory.

Zingales, L., 1998. Survival of the Fittest or the Fattest? Exit and Financing in the Trucking Industry. The Journal of Finance, 53(3), pp.905-938.

Zmijewski, M.E. and Hagerman, R.L., 1981. An income strategy approach to the positive theory of accounting standard setting/choice. Journal of accounting and Economics, 3(2), pp.129-149. 
TABLE 1.1

Descriptive Statistics

\begin{tabular}{|c|c|c|c|c|c|c|}
\hline Variables & Observations & Mean & S.D. & $1^{\text {st }}$ quartile & Median & $3^{\text {rd }}$ quartile \\
\hline FINLEV & 23,584 & 0.22 & 0.18 & 0.06 & 0.21 & 0.34 \\
\hline$B A N K D E B T$ & 9,638 & 0.09 & 0.12 & 0.00 & 0.03 & 0.13 \\
\hline NONBANKDEBT & 9,178 & 0.19 & 0.15 & 0.05 & 0.17 & 0.28 \\
\hline$Z L$ & 23,584 & 0.12 & 0.32 & 0.00 & 0.00 & 0.00 \\
\hline$A C C U R A C Y$ & 23,584 & 6.31 & 1.45 & 5.40 & 6.40 & 7.36 \\
\hline DISPERSION & 23,584 & 0.47 & 0.99 & 0.06 & 0.15 & 0.41 \\
\hline$I D V O L$ & 21,437 & 0.05 & 0.02 & 0.03 & 0.04 & 0.06 \\
\hline SYNCH & 21,502 & -0.64 & 1.10 & -1.37 & -0.63 & 0.11 \\
\hline$P I N$ & 6,461 & 0.16 & 0.05 & 0.12 & 0.15 & 0.19 \\
\hline SIZE & 23,584 & 7.30 & 1.75 & 6.07 & 7.20 & 8.42 \\
\hline$M B$ & 23,584 & 3.53 & 3.83 & 1.53 & 2.42 & 3.97 \\
\hline HORIZON & 23,584 & 3.81 & 0.38 & 3.59 & 3.85 & 4.08 \\
\hline$C h \_E P S$ & 23,584 & 0.00 & 0.08 & -0.01 & 0.01 & 0.02 \\
\hline LOSS & 23,584 & 0.20 & 0.40 & 0.00 & 0.00 & 0.00 \\
\hline$S T D V \_R O A$ & 23,58 & 0.08 & 0.13 & 0.02 & 0.04 & 0.08 \\
\hline$N \_A N A L Y S T$ & 23,584 & 2.02 & 0.54 & 1.61 & 1.95 & 2.40 \\
\hline INST_HOLDING & 23,584 & 0.65 & 0.23 & 0.50 & 0.68 & 0.84 \\
\hline OPLLEV & 23,584 & 0.28 & 0.12 & 0.19 & 0.26 & 0.35 \\
\hline TANGIBILITY & 23,548 & 0.31 & 0.24 & 0.12 & 0.25 & 0.46 \\
\hline BIG4 & 23,584 & 0.74 & 0.44 & 0.00 & 1.00 & 1.00 \\
\hline DIVPAYER & 23,584 & 0.48 & 0.50 & 0.00 & 0.00 & 1.00 \\
\hline$S T D \_C F O$ & 22,927 & 0.08 & 0.10 & 0.03 & 0.05 & 0.09 \\
\hline$A C C R U A L S$ & 23,584 & -0.07 & 0.09 & -0.10 & -0.06 & -0.02 \\
\hline TRVOL & 23,584 & 18.49 & 1.60 & 17.36 & 18.49 & 19.59 \\
\hline SP500 & 23,584 & 0.30 & 0.46 & 0.00 & 0.00 & 1.00 \\
\hline HERFIN & 23,584 & 6.54 & 0.56 & 6.16 & 6.45 & 6.83 \\
\hline NFIRM & 23,584 & 5.35 & 0.91 & 4.76 & 5.47 & 5.96 \\
\hline ROACORR & 22,668 & 0.22 & 0.40 & -0.08 & 0.23 & 0.54 \\
\hline
\end{tabular}


TABLE 1.2

Correlation Matrix

\begin{tabular}{|c|c|c|c|c|c|c|c|c|c|c|}
\hline & FINLEV & BANKDEBT & $\begin{array}{c}\text { NONBANK } \\
D E B T\end{array}$ & ACCURACY & $\begin{array}{l}\text { DISPE } \\
\text { RSION }\end{array}$ & SIZE & $M B$ & HORIZON & Ch_EPS & LOSS \\
\hline FINLEV & 1 & & & & & & & & & \\
\hline BANKDEBT & 0.4892 & 1 & & & & & & & & \\
\hline NONBANKDEBT & 0.7521 & -0.2027 & 1 & & & & & & & \\
\hline ACCURACY & -0.1245 & -0.1307 & -0.0416 & 1 & & & & & & \\
\hline DISPERSION & 0.1667 & 0.1005 & 0.1113 & -0.4827 & 1 & & & & & \\
\hline SIZE & -0.0509 & -0.3166 & 0.1812 & 0.4626 & -0.2864 & 1 & & & & \\
\hline$M B$ & 0.0825 & 0.0282 & 0.0674 & 0.1753 & -0.1028 & 0.1918 & 1 & & & \\
\hline HORIZON & -0.0324 & 0.0209 & -0.0526 & -0.0377 & -0.0012 & -0.0972 & 0.0266 & 1 & & \\
\hline$C h \_E P S$ & -0.0016 & 0.0153 & -0.0122 & -0.0067 & 0.0704 & 0.0015 & 0.0045 & 0.0066 & 1 & \\
\hline LOSS & 0.1306 & 0.0723 & 0.0929 & -0.4096 & 0.4238 & -0.3199 & -0.0448 & 0.0402 & 0.0273 & 1 \\
\hline$S T D V \_R O A$ & -0.0641 & 0.0216 & -0.0883 & -0.2306 & 0.1833 & -0.2483 & 0.1307 & 0.0478 & -0.0391 & 0.3522 \\
\hline$N \_A N A L Y S T$ & -0.0004 & -0.1797 & 0.1347 & 0.102 & -0.0253 & 0.4322 & 0.0035 & -0.0939 & 0.0001 & -0.053 \\
\hline INST_HOLDING & -0.0315 & -0.0153 & -0.0237 & 0.1424 & -0.1344 & 0.0894 & 0.0031 & -0.0274 & 0.0191 & -0.1223 \\
\hline OPLLEV & -0.2650 & -0.2238 & -0.1296 & 0.0427 & -0.0452 & 0.1926 & 0.1568 & -0.0228 & 0.0073 & -0.0915 \\
\hline TANGIBILITY & 0.1866 & 0.0611 & 0.1635 & -0.1379 & 0.1138 & -0.0772 & -0.1272 & -0.1569 & -0.0037 & 0.0628 \\
\hline BIG4 & 0.0579 & 0.0272 & 0.0443 & 0.0166 & -0.0155 & -0.0111 & -0.0169 & -0.0346 & -0.002 & -0.0032 \\
\hline DIVPAYER & -0.0075 & -0.1744 & 0.1216 & 0.2211 & -0.156 & 0.4315 & -0.0094 & -0.0528 & 0.0283 & -0.2464 \\
\hline$S T D \_C F O$ & -0.1116 & 0.0149 & -0.1375 & -0.2006 & 0.1111 & -0.2711 & 0.1600 & 0.0557 & -0.0212 & 0.2756 \\
\hline ACCRUALS & -0.0330 & -0.0314 & -0.0135 & 0.1722 & -0.1916 & 0.1475 & -0.0147 & 0.0284 & -0.0852 & -0.3997 \\
\hline
\end{tabular}




\begin{tabular}{|c|c|c|c|c|c|c|c|c|c|}
\hline & $S T D V \_R O A$ & N_ANALYST & $\begin{array}{c}I N S T_{-} H O L \\
D I N G\end{array}$ & OPLLEV & $\begin{array}{l}\text { TANGI } \\
\text { BILITYY }\end{array}$ & BIG4 & $\begin{array}{c}\text { DIVPA } \\
\text { YER }\end{array}$ & $S T D \_C F O$ & $\begin{array}{c}\text { ACCRU } \\
A L S\end{array}$ \\
\hline$S T D V_{-} R O A$ & 1 & & & & & & & & \\
\hline$N \_A N A L Y S T$ & -0.0713 & 1 & & & & & & & \\
\hline INST_HOLDING & -0.1753 & 0.0415 & 1 & & & & & & \\
\hline OPLLEV & -0.1284 & 0.0365 & 0.0242 & 1 & & & & & \\
\hline TANGIBILITY & -0.0546 & 0.2778 & -0.0941 & -0.1055 & 1 & & & & \\
\hline$B I G 4$ & -0.0511 & 0.0062 & 0.042 & 0.028 & 0.0445 & 1 & & & \\
\hline DIVPAYER & -0.2809 & 0.1673 & -0.006 & 0.2343 & 0.0136 & 0.0498 & 1 & & \\
\hline$S T D \_C F O$ & 0.7729 & -0.0787 & -0.1689 & -0.0905 & -0.046 & -0.032 & -0.2652 & 1 & \\
\hline ACCRUALS & -0.2275 & -0.1047 & 0.0455 & 0.0358 & -0.3156 & -0.0047 & 0.1616 & -0.1726 & 1 \\
\hline
\end{tabular}


TABLE 1.3 (a)

Regression Analyses of Forecast Accuracy and Forecast Dispersion on Financial Leverage

\begin{tabular}{|c|c|c|c|c|c|c|c|c|}
\hline & \multicolumn{4}{|c|}{ Dep Var: FORECAST ACCURACY } & \multicolumn{4}{|c|}{ Dep Var: FORECAST DISPERSION } \\
\hline VARIABLES & Coefficient & $t$-stat & Coefficients & $t$-stat & Coefficients & $t$-stat & Coefficients & $t$-stat \\
\hline FINLEV & $-0.869 * * *$ & -12.703 & $-1.031 * * *$ & -14.977 & $0.683 * * *$ & 12.738 & $0.741 * * *$ & 13.552 \\
\hline$S I Z E$ & $0.326 * * *$ & 32.599 & $0.339 * * *$ & 32.341 & $-0.118 * * *$ & -19.132 & $-0.126^{* * *}$ & -18.980 \\
\hline$M B$ & $0.033 * * *$ & 11.928 & $0.038 * * *$ & 13.540 & $-0.013 * * *$ & -7.938 & $-0.015^{* * *}$ & -9.234 \\
\hline HORIZON & $-0.045^{*}$ & -1.930 & $-0.051 * *$ & -2.176 & $-0.027 *$ & -1.761 & -0.022 & -1.400 \\
\hline$C h \_E P S$ & -0.007 & -0.059 & -0.068 & -0.621 & $0.907 * * *$ & 6.259 & $0.836 * * *$ & 5.828 \\
\hline$L \overline{O S S}$ & $-0.881 * * *$ & -32.391 & $-0.852 * * *$ & -30.657 & $0.788 * * *$ & 26.805 & $0.739 * * *$ & 25.902 \\
\hline$S T D V \_R O A$ & $-0.721 * * *$ & -8.263 & $-0.777 * * *$ & -6.235 & $0.281 * * *$ & 3.681 & $0.515^{* * *}$ & 4.424 \\
\hline$N \_A N \bar{A} L Y S T$ & $-0.160 * * *$ & -7.236 & $-0.143 * * *$ & -6.574 & $0.110 * * *$ & 7.388 & $0.099 * * *$ & 6.715 \\
\hline$\overline{O P L L E V}$ & & & $-1.410 * * *$ & -14.429 & & & $0.740 * * *$ & 10.270 \\
\hline TANGIBILITY & & & $-0.207 * * *$ & -2.809 & & & $0.112 * *$ & 2.155 \\
\hline$B I G 4$ & & & $0.053 * *$ & 1.984 & & & -0.015 & -0.898 \\
\hline DIVPAYER & & & $0.052 * *$ & 1.995 & & & $-0.042 * *$ & -2.557 \\
\hline$S T D \_C F O$ & & & -0.258 & -1.621 & & & $-0.248 *$ & -1.695 \\
\hline$A C \overline{C R} U A L S$ & & & $-0.305 * * *$ & -2.701 & & & 0.011 & 0.102 \\
\hline Constant & $4.328 * * *$ & 21.515 & $4.656^{* * *} *$ & 20.614 & $1.026 * * *$ & 9.528 & $0.934 * * *$ & 7.182 \\
\hline Year FE & Yes & & Yes & & Yes & & Yes & \\
\hline Industry FE & Yes & & Yes & & Yes & & Yes & \\
\hline Observations & 23,584 & & 22,892 & & 23,584 & & 22,892 & \\
\hline R-squared & 0.335 & & 0.347 & & 0.248 & & 0.252 & \\
\hline
\end{tabular}

Sample includes firm-year observations with at least three unique analyst forecasts from 1989 to 2017 . The variable of interest, FINLEV, is defined as the ratio of financial debt $(D L C+D L T T)$ to total assets $(A T)$. All other variables in the table are defined in Appendix A. Industry dummies capture industry fixed effects with two-digit SIC code classifications. Standard errors are robust and clustered by firm (gvkey). Continuous variables are winsorized at top and bottom 1 percent. $* * *, * *$, and $*$ respectively denote significance at $1 \%, 5 \%$, and $10 \%$. 
TABLE 1.3 (b)

Regression Analyses of Firm-specific Information Environment on Financial Leverage

\begin{tabular}{|c|c|c|c|c|c|c|c|c|c|c|c|c|}
\hline \multirow[b]{2}{*}{ VARIABLES } & \multicolumn{4}{|c|}{ Dep Var: IDVOL } & \multicolumn{4}{|c|}{ Dep Var: SYNCH } & \multicolumn{4}{|c|}{ Dep Var: PIN } \\
\hline & Coeff & $t$-stat & Coeff & $t$-stat & Coeff & $t$-stat & Coeff & $t$-stat & Coeff & $t$-stat & Coeff & $t$-stat \\
\hline FINLEV & $-0.005 * * *$ & -5.288 & $-0.004 * * *$ & -4.380 & -0.017 & -0.313 & -0.054 & -0.969 & 0.003 & 0.721 & 0.003 & 0.733 \\
\hline$S I Z E$ & $-0.011 * * *$ & -60.582 & $-0.011 * * *$ & -59.728 & $0.216 * * *$ & 19.937 & $0.221 * * *$ & 20.256 & $-0.011 * * *$ & -15.670 & $-0.011 * * *$ & -15.479 \\
\hline OPLEV & $-0.005 * * *$ & -3.985 & $-0.005 * * *$ & -3.615 & -0.011 & -0.140 & -0.017 & -0.217 & -0.004 & -0.772 & -0.004 & -0.781 \\
\hline$T R V O L$ & $0.008 * * *$ & 40.583 & $0.008 * * *$ & 39.913 & $0.093 * * *$ & 8.319 & $0.086 * * *$ & 7.496 & $-0.010 * * *$ & -11.934 & $-0.010 * * *$ & -11.724 \\
\hline$N \_A N A L Y S T$ & $-0.001 * * *$ & -3.592 & $-0.001 * * *$ & -3.512 & $0.097 * * *$ & 5.178 & $0.090 * * *$ & 4.758 & $-0.003 * * *$ & -2.693 & $-0.003 * *$ & -2.404 \\
\hline SP500 & $-0.005 * * *$ & -11.594 & $-0.005 * * *$ & -10.958 & 0.034 & 1.257 & 0.013 & 0.493 & $-0.007 * * *$ & -5.152 & $-0.007 * * *$ & -5.110 \\
\hline$S T D V \_R O A$ & $0.030 * * *$ & 23.017 & $0.029 * * *$ & 20.488 & -0.086 & -1.428 & -0.076 & -1.152 & $0.046 * * *$ & 4.043 & $0.048 * * *$ & 3.826 \\
\hline HERFIN & & & $0.001 *$ & 1.908 & & & -0.022 & -0.699 & & & $0.007 * * *$ & 2.664 \\
\hline NFIRM & & & $0.007 * * *$ & 9.574 & & & $-0.233 * * *$ & -4.742 & & & $0.017 * * *$ & 3.119 \\
\hline ROACORR & & & $-0.001 * *$ & -2.124 & & & $0.165 * * *$ & 8.202 & & & 0.001 & 0.772 \\
\hline Constant & $-0.025 * * *$ & -7.747 & $-0.058 * * *$ & -9.399 & $-3.724 * * *$ & -12.232 & $-2.674 * * *$ & -5.719 & $0.429 * * *$ & 28.253 & $0.316 * * *$ & 8.516 \\
\hline Year FE & Yes & & Yes & & Yes & & Yes & & Yes & & Yes & \\
\hline Industry FE & Yes & & Yes & & Yes & & Yes & & Yes & & Yes & \\
\hline Observations & 21,437 & & 20,590 & & 21,502 & & 20,652 & & 6,461 & & 6,304 & \\
\hline R-squared & 0.674 & & 0.670 & & 0.408 & & 0.417 & & 0.550 & & 0.553 & \\
\hline
\end{tabular}

Sample includes firm-year observations with at least three unique analyst forecasts from 1989 to 2017. The variable of interest, FINLEV, is defined as the ratio of financial debt $(D L C+D L T T)$ to total assets $(A T)$. All other variables in the table are defined in Appendix A. Industry dummies capture industry fixed effects with two-digit SIC code classifications. Standard errors are robust and clustered by firm (gvkey). Continuous variables are winsorized at top and bottom 1 percent. $* * *, * *$, and * respectively denote significance at $1 \%, 5 \%$, and $10 \%$. 
TABLE 1.4 (a)

Regression Analyses of Forecast Accuracy and Forecast Dispersion on Leverage Decile

\begin{tabular}{|c|c|c|c|c|c|c|c|c|}
\hline & \multicolumn{4}{|c|}{ Dep Var: FORECAST ACCURACY } & \multicolumn{4}{|c|}{ Dep Var: FORECAST DISPERSION } \\
\hline VARIABLES & Coefficient & $t$-stat & Coefficients & $t$-stat & Coefficients & $t$-stat & Coefficients & $t$-stat \\
\hline$L E V \_D E C$ & $-0.052 * * *$ & -12.741 & $-0.059 * * *$ & -14.487 & $0.039 * * *$ & 13.461 & $0.041 * * *$ & 13.863 \\
\hline SIZE & $0.329 * * *$ & 32.792 & $0.342 * * *$ & 32.428 & $-0.121 * * *$ & -19.436 & $-0.129 * * *$ & -19.210 \\
\hline$M B$ & $0.031 * * *$ & 11.380 & $0.036 * * *$ & 12.753 & $-0.012 * * *$ & -7.373 & $-0.014 * * *$ & -8.482 \\
\hline HORIZON & $-0.046^{*}$ & -1.954 & $-0.051 * *$ & -2.182 & $-0.027 *$ & -1.746 & -0.022 & -1.398 \\
\hline$C h \_E P S$ & -0.013 & -0.116 & -0.073 & -0.669 & $0.911 * * *$ & 6.270 & $0.839 * * *$ & 5.832 \\
\hline LOSS & $-0.887 * * *$ & -32.692 & $-0.861 * * *$ & -31.028 & $0.794 * * *$ & 26.942 & $0.747 * * *$ & 26.030 \\
\hline$S T D V \_R O A$ & $-0.720 * * *$ & -8.280 & $-0.766^{* * *}$ & -6.134 & $0.275 * * *$ & 3.626 & $0.505^{* * *} *$ & 4.342 \\
\hline$N \_A N \bar{A} L Y S T$ & $-0.160 * * *$ & -7.216 & $-0.143 * * *$ & -6.531 & $0.109 * * *$ & 7.376 & $0.099 * * *$ & 6.677 \\
\hline$\overline{O P} L L E V$ & & & $-1.345 * * *$ & -13.859 & & & $0.690 * * *$ & 9.578 \\
\hline TANGIBILITY & & & $-0.210 * * *$ & -2.838 & & & $0.117 * *$ & 2.235 \\
\hline BIG4 & & & $0.051 *$ & 1.903 & & & -0.013 & -0.798 \\
\hline DIVPAYER & & & $0.053 * *$ & 2.031 & & & $-0.043 * * *$ & -2.577 \\
\hline$S T D \_C F O$ & & & -0.250 & -1.563 & & & $-0.258 *$ & -1.755 \\
\hline$A C C R U A L S$ & & & $-0.296 * * *$ & -2.621 & & & 0.005 & 0.051 \\
\hline Constant & $4.408 * * *$ & 21.876 & $4.736 * * *$ & 20.991 & $0.972 * * *$ & 9.077 & $0.883 * * *$ & 6.833 \\
\hline Year FE & Yes & & Yes & & Yes & & Yes & \\
\hline Industry FE & Yes & & Yes & & Yes & & Yes & \\
\hline Observations & 23,584 & & 22,892 & & 23,584 & & 22,892 & \\
\hline R-squared & 0.335 & & 0.346 & & 0.246 & & 0.250 & \\
\hline
\end{tabular}

Sample includes firm-year observations with at least three unique analyst forecasts from 1989 to 2017 . The variable of interest, $L E V \_D E C$, is financial leverage ranked every year in deciles, and then transformed to a continues variable. All other variables in the table are defined in Appendix A. Industry dummies capture industry fixed effects with twodigit SIC code classifications. Standard errors are robust and clustered by firm ( $g v k e y)$. Continuous variables are winsorized at top and bottom 1 percent. $* * *, * *$, and $*$ respectively denote significance at $1 \%, 5 \%$, and $10 \%$. 
TABLE 1.4 (b)

Regression Analyses of Firm-specific Information Environment on Leverage Decile

\begin{tabular}{|c|c|c|c|c|c|c|c|c|c|c|c|c|}
\hline & \multicolumn{4}{|c|}{ Dep Var: IDVOL } & \multicolumn{4}{|c|}{ Dep Var: SYNCH } & \multicolumn{4}{|c|}{ Dep Var: PIN } \\
\hline VARIABLES & Coeff & $t$-stat & Coeff & $t$-stat & Coeff & $t$-stat & Coeff & $t$-stat & Coeff & $t$-stat & Coeff & $t$-stat \\
\hline$L E V \_D E C$ & $-0.000 * * *$ & -6.665 & $-0.000 * * *$ & -5.746 & -0.001 & -0.170 & -0.003 & -0.801 & 0.000 & 0.310 & 0.000 & 0.301 \\
\hline$S I Z \bar{E}$ & $-0.011 * * *$ & -60.793 & $-0.011 * * *$ & -59.962 & $0.216 * * *$ & 20.034 & $0.221 * * *$ & 20.383 & $-0.011 * * *$ & -15.798 & $-0.011 * * *$ & -15.620 \\
\hline$O P L E V$ & $-0.005 * * *$ & -4.000 & $-0.005 * * *$ & -3.647 & -0.009 & -0.113 & -0.013 & -0.167 & -0.005 & -0.894 & -0.005 & -0.908 \\
\hline$T R V O L$ & $0.008 * * *$ & 40.754 & $0.008 * * *$ & 40.089 & $0.093 * * *$ & 8.321 & $0.085 * * *$ & 7.488 & $-0.010 * * *$ & -11.910 & $-0.010 * * *$ & -11.702 \\
\hline N_ANALYST & $-0.001 * * *$ & -3.543 & $-0.001 * * *$ & -3.470 & $0.097 * * *$ & 5.178 & $0.090 * * *$ & 4.764 & $-0.003 * * *$ & -2.682 & $-0.003 * *$ & -2.395 \\
\hline$S \bar{P} 500$ & $-0.005 * * *$ & -11.553 & $-0.005 * * *$ & -10.924 & 0.034 & 1.253 & 0.013 & 0.491 & $-0.007 * * *$ & -5.138 & $-0.007 * * *$ & -5.096 \\
\hline$S T D V \_R O A$ & $0.030 * * *$ & 22.831 & $0.028 * * *$ & 20.307 & -0.085 & -1.402 & -0.075 & -1.135 & $0.046 * * *$ & 4.012 & $0.048 * * *$ & 3.794 \\
\hline HERFIN & & & $0.001 *$ & 1.896 & & & -0.022 & -0.688 & & & $0.007 * * *$ & 2.646 \\
\hline NFIRM & & & $0.007 * * *$ & 9.587 & & & $-0.233 * * *$ & -4.736 & & & $0.017 * * *$ & 3.109 \\
\hline ROACORR & & & $-0.001 * *$ & -2.038 & & & $0.165 * * *$ & 8.190 & & & 0.001 & 0.812 \\
\hline Constant & $-0.024 * * *$ & -7.459 & $-0.057 * * *$ & -9.252 & $-3.723 * * *$ & -12.216 & $-2.673 * * *$ & -5.709 & $0.429 * * *$ & 28.378 & $0.316^{* * *}$ & 8.530 \\
\hline Year FE & Yes & & Yes & & Yes & & Yes & & Yes & & Yes & \\
\hline Industry FE & Yes & & Yes & & Yes & & Yes & & Yes & & Yes & \\
\hline Observations & 21,437 & & 20,590 & & 21,502 & & 20,652 & & 6,461 & & 6,304 & \\
\hline R-squared & 0.674 & & 0.671 & & 0.408 & & 0.417 & & 0.550 & & 0.553 & \\
\hline
\end{tabular}

Sample includes firm-year observations with at least three unique analyst forecasts from 1989 to 2017. The variable of interest, $L E V \_D E C$, is financial leverage ranked every year in deciles, and then transformed to a continues variable. All other variables in the table are defined in Appendix A. Industry dummies capture industry fixed effects with twodigit SIC code classifications. Standard errors are robust and clustered by firm ( $g v k e y)$. Continuous variables are winsorized at top and bottom 1 percent. $* * * * *$, and * respectively denote significance at $1 \%, 5 \%$, and $10 \%$. 
TABLE 1.5 (a)

Regression Analyses of Forecast Accuracy and Forecast Dispersion on Leverage Tercile

\begin{tabular}{|c|c|c|c|c|c|c|c|c|}
\hline & \multicolumn{4}{|c|}{ Dep Var: FORECAST ACCURACY } & \multicolumn{4}{|c|}{ Dep Var: FORECAST DISPERSION } \\
\hline VARIABLES & Coefficient & $t$-stat & Coefficients & $t$-stat & Coefficients & $t$-stat & Coefficients & $t$-stat \\
\hline$L O W$ & $0.323 * * *$ & 11.067 & $0.365 * * *$ & 12.425 & $-0.244 * * *$ & -12.182 & $-0.255^{* * *}$ & -12.214 \\
\hline$S I Z E$ & $0.339 * * *$ & 28.118 & $0.348 * * *$ & 27.827 & $-0.137 * * *$ & -17.102 & $-0.140 * * *$ & -16.192 \\
\hline$M B$ & $0.027 * * *$ & 8.947 & $0.031 * * *$ & 10.029 & $-0.009 * * *$ & -4.922 & $-0.012 * * *$ & -5.893 \\
\hline HORIZON & $-0.091 * * *$ & -3.285 & $-0.099 * * *$ & -3.574 & -0.021 & -1.030 & -0.013 & -0.634 \\
\hline$C h \_E P S$ & -0.162 & -1.247 & $-0.228 *$ & -1.769 & $0.869 * * *$ & 4.735 & $0.804 * * *$ & 4.422 \\
\hline LOSS & $-0.915 * * *$ & -30.156 & $-0.904 * * *$ & -28.762 & $0.831 * * *$ & 23.576 & $0.792 * * *$ & 23.072 \\
\hline$S T D V \_R O A$ & $-0.546 * * *$ & -5.830 & $-0.586 * * *$ & -4.281 & $0.242 * * *$ & 2.997 & $0.436 * * *$ & 3.500 \\
\hline$N \_A N A L Y S T$ & $-0.151 * * *$ & -5.810 & $-0.133 * * *$ & -5.156 & $0.126 * * *$ & 6.499 & $0.114 * * *$ & 5.858 \\
\hline$\overline{O P L L E V}$ & & & $-1.273 * * *$ & -11.687 & & & $0.674 * * *$ & 7.694 \\
\hline TANGIBILITY & & & $-0.216^{* *}$ & -2.575 & & & $0.177 * * *$ & 2.661 \\
\hline$B I G 4$ & & & 0.035 & 1.187 & & & -0.016 & -0.747 \\
\hline DIVPAYER & & & $0.062 * *$ & 2.084 & & & $-0.072 * * *$ & -3.395 \\
\hline$S T D \_C F O$ & & & -0.197 & -1.152 & & & -0.218 & -1.316 \\
\hline$A C C R U A L S$ & & & $-0.360 * * *$ & -2.762 & & & 0.088 & 0.692 \\
\hline Constant & $3.879 * * *$ & 17.148 & $4.226 * * *$ & 16.207 & $1.438 * * *$ & 10.971 & $1.379 * * *$ & 8.306 \\
\hline Year FE & Yes & & Yes & & Yes & & Yes & \\
\hline Industry FE & Yes & & Yes & & Yes & & Yes & \\
\hline Observations & 15,714 & & 15,241 & & 15,714 & & 15,241 & \\
\hline R-squared & 0.345 & & 0.355 & & 0.252 & & 0.256 & \\
\hline
\end{tabular}

Sample includes firm-year observations with at least three unique analyst forecasts from 1989 to 2017. The variable of interest, $L O W$, is an indicator variable 1 for bottom tercile observations based on financial leverage ranked every year, and 0 for top tercile observations. All other variables in the table are defined in Appendix A. Industry dummies capture industry fixed effects with two-digit SIC code classifications. Standard errors are robust and clustered by firm (gvkey). Continuous variables are winsorized at top and bottom 1 percent. ***,**, and * respectively denote significance at $1 \%, 5 \%$, and $10 \%$. 
TABLE 1.5 (b)

Regression Analyses of Firm-specific Information Environment on Leverage Tercile

\begin{tabular}{|c|c|c|c|c|c|c|c|c|c|c|c|c|}
\hline \multirow[b]{2}{*}{ VARIABLES } & \multicolumn{4}{|c|}{ Dep Var: IDVOL } & \multicolumn{4}{|c|}{ Dep Var: SYNCH } & \multicolumn{4}{|c|}{ Dep Var: PIN } \\
\hline & Coeff & $t$-stat & Coeff & $t$-stat & Coeff & $t$-stat & Coeff & $t$-stat & Coeff & $t$-stat & Coeff & $t$-stat \\
\hline$L O W$ & $0.003 * * *$ & 7.515 & $0.003 * * *$ & 6.953 & -0.021 & -0.883 & -0.010 & -0.407 & -0.001 & -0.855 & -0.001 & -0.797 \\
\hline SIZE & $-0.011 * * *$ & -52.732 & $-0.011 * * *$ & -51.663 & $0.195 * * *$ & 16.940 & $0.201 * * *$ & 17.032 & $-0.011 * * *$ & -13.304 & $-0.011 * * *$ & -13.042 \\
\hline OPLEV & $-0.005 * * *$ & -3.148 & $-0.005^{* * *}$ & -2.844 & -0.048 & -0.557 & -0.048 & -0.545 & -0.004 & -0.581 & -0.005 & -0.812 \\
\hline TRVOL & $0.008 * * *$ & 36.078 & $0.008 * * *$ & 35.319 & $0.106 * * *$ & 8.630 & $0.097 * * *$ & 7.761 & $-0.010 * * *$ & -10.190 & $-0.010 * * *$ & -10.069 \\
\hline$N \_A N A L Y S T$ & $-0.001 * * *$ & -3.837 & $-0.001 * * *$ & -3.699 & $0.111 * * *$ & 5.178 & $0.105 * * *$ & 4.806 & $-0.003 * *$ & -2.090 & $-0.002 *$ & -1.711 \\
\hline$S \bar{P} 500$ & $-0.005 * * *$ & -9.610 & $-0.005 * * *$ & -8.859 & $0.056^{*}$ & 1.757 & 0.031 & 0.982 & $-0.009 * * *$ & -5.006 & $-0.009 * * *$ & -4.811 \\
\hline$S T D V \_R O A$ & $0.028 * * *$ & 19.853 & $0.026 * * *$ & 17.294 & -0.107 & -1.627 & -0.116 & -1.578 & $0.037 * * *$ & 2.905 & $0.040 * * *$ & 2.738 \\
\hline HERFIN & & & 0.001 & 1.464 & & & 0.001 & 0.035 & & & 0.003 & 0.954 \\
\hline NFIRM & & & $0.007 * * *$ & 7.798 & & & $-0.163 * * *$ & -2.930 & & & 0.009 & 1.262 \\
\hline ROACORR & & & -0.001 & -1.610 & & & $0.165 * * *$ & 7.193 & & & -0.000 & -0.075 \\
\hline Constant & $-0.034 * * *$ & -9.401 & $-0.063 * * *$ & -9.041 & $-3.968 * * *$ & -9.595 & $-3.358 * * *$ & -6.030 & $0.424 * * *$ & 24.233 & $0.370 * * *$ & 8.030 \\
\hline Year FE & Yes & & Yes & & Yes & & Yes & & Yes & & Yes & \\
\hline Industry FE & Yes & & Yes & & Yes & & Yes & & Yes & & Yes & \\
\hline Observations & 14,260 & & 13,596 & & 14,308 & & 13,641 & & 3,949 & & 3,849 & \\
\hline R-squared & 0.663 & & 0.658 & & 0.387 & & 0.395 & & 0.546 & & 0.547 & \\
\hline
\end{tabular}

Sample includes firm-year observations with at least three unique analyst forecasts from 1989 to 2017. The variable of interest, $L O W$, is an indicator variable 1 for bottom tercile observations based on financial leverage ranked every year, and 0 for top tercile observations. All other variables in the table are defined in Appendix A. Industry dummies capture industry fixed effects with two-digit SIC code classifications. Standard errors are robust and clustered by firm (gvkey). Continuous variables are winsorized at top and bottom 1 percent. ***, **, and * respectively denote significance at $1 \%, 5 \%$, and $10 \%$. 
TABLE 1.6 (a)

Regression Analyses of Forecast Accuracy and Forecast Dispersion on Zero Leverage

\begin{tabular}{|c|c|c|c|c|c|c|c|c|}
\hline & \multicolumn{4}{|c|}{ Dep Var: FORECAST ACCURACY } & \multicolumn{4}{|c|}{ Dep Var: FORECAST DISPERSION } \\
\hline VARIABLES & Coefficient & $t$-stat & Coefficients & $t$-stat & Coefficients & $t$-stat & Coefficients & $t$-stat \\
\hline$Z L$ & $0.259 * * *$ & 9.074 & $0.230 * * *$ & 8.101 & $-0.188 * * *$ & -10.123 & $-0.165 * * *$ & -8.837 \\
\hline$S I Z E$ & $0.333 * * *$ & 32.988 & $0.343 * * *$ & 32.391 & $-0.124 * * *$ & -19.614 & $-0.130 * * *$ & -19.288 \\
\hline$M B$ & $0.029 * * *$ & 10.473 & $0.033 * * *$ & 11.417 & $-0.010 * * *$ & -6.449 & $-0.011 * * *$ & -7.059 \\
\hline HORIZON & $-0.041 *$ & -1.753 & $-0.046^{* *}$ & -1.968 & $-0.031 * *$ & -1.976 & -0.025 & -1.601 \\
\hline$C h \_E P S$ & 0.009 & 0.083 & -0.054 & -0.489 & $0.893 * * *$ & 6.106 & $0.826 * * *$ & 5.698 \\
\hline LOSS & $-0.925 * * *$ & -34.085 & $-0.913 * * *$ & -32.699 & $0.823 * * *$ & 27.369 & $0.783 * * *$ & 26.481 \\
\hline$S T D V \_R O A$ & $-0.610 * * *$ & -7.153 & $-0.709 * * *$ & -5.635 & $0.189 * *$ & 2.516 & $0.466 * * *$ & 3.968 \\
\hline$N \_A N \bar{A} L Y S T$ & $-0.166^{* * *}$ & -7.426 & $-0.146^{* * *}$ & -6.607 & $0.114^{* * *}$ & 7.650 & $0.101 * * *$ & 6.804 \\
\hline$\overline{O P} L L E V$ & & & $-1.133 * * *$ & -11.533 & & & $0.541 * * *$ & 7.518 \\
\hline TANGIBILITY & & & $-0.300 * * *$ & -3.974 & & & $0.179 * * *$ & 3.367 \\
\hline BIG4 & & & 0.042 & 1.561 & & & -0.007 & -0.430 \\
\hline DIVPAYER & & & 0.039 & 1.466 & & & $-0.033 * *$ & -1.989 \\
\hline$S T D \_C F O$ & & & -0.100 & -0.621 & & & $-0.361 * *$ & -2.427 \\
\hline$A C C R U A L S$ & & & $-0.336 * * *$ & -2.965 & & & 0.033 & 0.312 \\
\hline Constant & $4.062 * * *$ & 20.918 & $4.358 * * *$ & 19.846 & $1.235 * * *$ & 12.499 & $1.148 * * *$ & 9.486 \\
\hline Year FE & Yes & & Yes & & Yes & & Yes & \\
\hline Industry FE & Yes & & Yes & & Yes & & Yes & \\
\hline Observations & 23,584 & & 22,892 & & 23,584 & & 22,892 & \\
\hline R-squared & 0.329 & & 0.338 & & 0.239 & & 0.242 & \\
\hline
\end{tabular}

Sample includes firm-year observations with at least three unique analyst forecasts from 1989 to 2017. The variable of interest, $Z L$, is an indicator variable 1 for firm-year observations with zero financial leverage, 0 otherwise. All other variables in the table are defined in Appendix A. Industry dummies capture industry fixed effects with two-digit SIC code classifications. Standard errors are robust and clustered by firm (gvkey). Continuous variables are winsorized at top and bottom 1 percent. $* * *, * *$, and * respectively denote significance at $1 \%, 5 \%$, and $10 \%$. 
TABLE 1.6 (b)

Regression Analyses of Firm-specific Information Environment on Zero Leverage

\begin{tabular}{|c|c|c|c|c|c|c|c|c|c|c|c|c|}
\hline \multirow[b]{2}{*}{ VARIABLES } & \multicolumn{4}{|c|}{ Dep Var: IDVOL } & \multicolumn{4}{|c|}{ Dep Var: SYNCH } & \multicolumn{4}{|c|}{ Dep Var: PIN } \\
\hline & Coeff & $t$-stat & Coeff & $t$-stat & Coeff & $t$-stat & Coeff & $t$-stat & Coeff & $t$-stat & Coeff & $t$-stat \\
\hline$Z L$ & $0.001 * *$ & 2.290 & $0.001 *$ & 1.665 & 0.039 & 1.374 & $0.051 *$ & 1.727 & -0.004 & -1.234 & -0.004 & -1.191 \\
\hline OPLEV & $-0.004 * * *$ & -3.168 & $-0.004 * * *$ & -2.942 & -0.003 & -0.043 & 0.001 & 0.007 & -0.005 & -1.029 & -0.006 & -1.044 \\
\hline$T R V O L$ & $0.008 * * *$ & 40.213 & $0.008 * * *$ & 39.696 & $0.093 * * *$ & 8.430 & $0.085 * * *$ & 7.559 & $-0.010 * * *$ & -12.022 & $-0.010 * * *$ & -11.812 \\
\hline$N \_A N A L Y S T$ & $-0.001 * * *$ & -3.587 & $-0.001 * * *$ & -3.485 & $0.097 * * *$ & 5.169 & $0.089 * * *$ & 4.751 & $-0.003 * * *$ & -2.681 & $-0.003 * *$ & -2.401 \\
\hline$S \bar{P} 500$ & $-0.005 * * *$ & -11.657 & $-0.005 * * *$ & -11.025 & 0.035 & 1.294 & 0.014 & 0.518 & $-0.008 * * *$ & -5.174 & $-0.008 * * *$ & -5.133 \\
\hline$S T D V \_R O A$ & $0.031 * * *$ & 23.492 & $0.029 * * *$ & 20.844 & -0.092 & -1.534 & -0.080 & -1.204 & $0.046^{* * *}$ & 4.074 & $0.048 * * *$ & 3.834 \\
\hline HERFIN & & & $0.001 * *$ & 2.057 & & & -0.021 & -0.677 & & & $0.007 * * *$ & 2.674 \\
\hline NFIRM & & & $0.007 * * *$ & 9.649 & & & $-0.234 * * *$ & -4.771 & & & $0.017 * * *$ & 3.131 \\
\hline ROACORR & & & $-0.001 * *$ & -2.431 & & & $0.164 * * *$ & 8.143 & & & 0.001 & 0.832 \\
\hline Constant & $-0.026 * * *$ & -7.896 & $-0.059 * * *$ & -9.637 & $-3.734 * * *$ & -12.256 & $-2.694 * * *$ & -5.768 & $0.430 * * *$ & 28.480 & $0.317 * * *$ & 8.568 \\
\hline Year FE & Yes & & Yes & & Yes & & Yes & & Yes & & Yes & \\
\hline Industry FE & Yes & & Yes & & Yes & & Yes & & Yes & & Yes & \\
\hline
\end{tabular}

Sample includes firm-year observations with at least three unique analyst forecasts from 1989 to 2017. The variable of interest, $Z L$, is an indicator variable 1 for firm-year observations with zero financial leverage, 0 otherwise. All other variables in the table are defined in Appendix A. Industry dummies capture industry fixed effects with two-digit SIC code classifications. Standard errors are robust and clustered by firm (gvkey). Continuous variables are winsorized at top and bottom 1 percent. $* * *, * *$, and $*$ respectively denote significance at $1 \%, 5 \%$, and $10 \%$. 
TABLE 1.7 (a)

Regression Analyses of Forecast Accuracy and Forecast Dispersion on Financial Leverage (Excluding Zero Leverage)

\begin{tabular}{|c|c|c|c|c|c|c|c|c|}
\hline & \multicolumn{4}{|c|}{ Dep Var: FORECAST ACCURACY } & \multicolumn{4}{|c|}{ Dep Var: FORECAST DISPERSION } \\
\hline VARIABLES & Coefficient & $t$-stat & Coefficients & $t$-stat & Coefficients & $t$-stat & Coefficients & $t$-stat \\
\hline FINLEV & $-0.815 * * *$ & -10.748 & $-1.057 * * *$ & -13.733 & $0.634 * * *$ & 10.857 & $0.730 * * *$ & 12.206 \\
\hline$S I Z E$ & $0.320 * * *$ & 30.358 & $0.336 * * *$ & 30.094 & $-0.121 * * *$ & -17.936 & $-0.131 * * *$ & -18.012 \\
\hline$M B$ & $0.033 * * *$ & 10.971 & $0.039 * * *$ & 12.745 & $-0.015^{* * *}$ & -8.404 & $-0.017 * * *$ & -10.038 \\
\hline HORIZON & -0.036 & -1.402 & -0.037 & -1.466 & $-0.038 * *$ & -2.192 & $-0.034 *$ & -1.924 \\
\hline$C h \_E P S$ & 0.024 & 0.209 & -0.052 & -0.455 & $0.933 * * *$ & 6.054 & $0.863 * * *$ & 5.659 \\
\hline$L \overline{O S S}$ & $-0.901 * * *$ & -30.903 & $-0.869 * * *$ & -29.194 & $0.832 * * *$ & 25.659 & $0.778 * * *$ & 24.649 \\
\hline$S T D V \_R O A$ & $-0.844 * * *$ & -8.162 & $-1.000 * * *$ & -7.171 & $0.302 * * *$ & 3.156 & $0.649 * * *$ & 4.585 \\
\hline$N \_A N A L Y S T$ & $-0.172 * * *$ & -7.239 & $-0.155^{* * *} *$ & -6.628 & $0.115^{* * *}$ & 7.021 & $0.103 * * *$ & 6.402 \\
\hline$\overline{O P L L E V}$ & & & $-1.566 * * *$ & -14.238 & & & $0.839 * * *$ & 10.341 \\
\hline TANGIBILITY & & & $-0.230 * * *$ & -3.044 & & & $0.141 * *$ & 2.556 \\
\hline$B I G 4$ & & & $0.055^{*}$ & 1.916 & & & -0.015 & -0.841 \\
\hline DIVPAYER & & & $0.057 * *$ & 2.068 & & & $-0.045 * *$ & -2.503 \\
\hline$S T D \_C F O$ & & & -0.200 & -1.104 & & & $-0.397 * *$ & -2.320 \\
\hline$A C C R U A L S$ & & & $-0.395 * * *$ & -3.173 & & & 0.033 & 0.284 \\
\hline Constant & $4.335 * * *$ & 20.917 & $4.685 * * *$ & 20.049 & $1.087 * * *$ & 9.516 & $0.985 * * *$ & 7.123 \\
\hline Year FE & Yes & & Yes & & Yes & & Yes & \\
\hline Industry FE & Yes & & Yes & & Yes & & Yes & \\
\hline Observations & 20,847 & & 20,185 & & 20,847 & & 20,185 & \\
\hline R-squared & 0.338 & & 0.352 & & 0.253 & & 0.258 & \\
\hline
\end{tabular}

Sample includes firm-year observations with at least three unique analyst forecasts and non-zero financial leverage from 1989 to 2017. The variable of interest, FINLEV, is defined as the ratio of financial debt $(D L C+D L T T)$ to total assets $(A T)$. All other variables in the table are defined in Appendix A. Industry dummies capture industry fixed effects with two-digit SIC code classifications. Standard errors are robust and clustered by firm (gvkey). Continuous variables are winsorized at top and bottom 1 percent. $* * *, * *$, and $*$ respectively denote significance at $1 \%, 5 \%$, and $10 \%$. 
TABLE 1.7 (b)

Regression Analyses of Firm-specific Information Environment on Financial Leverage (Excluding Zero Leverage)

\begin{tabular}{|c|c|c|c|c|c|c|c|c|c|c|c|c|}
\hline \multirow[b]{2}{*}{ VARIABLES } & \multicolumn{4}{|c|}{ Dep Var: IDVOL } & \multicolumn{4}{|c|}{ Dep Var: SYNCH } & \multicolumn{4}{|c|}{ Dep Var: PIN } \\
\hline & Coeff & $t$-stat & Coeff & $t$-stat & Coeff & $t$-stat & Coeff & $t$-stat & Coeff & $t$-stat & Coeff & $t$-stat \\
\hline FINLEV & $-0.005 * * *$ & -4.834 & $-0.004 * * *$ & -4.155 & 0.010 & 0.175 & -0.023 & -0.392 & 0.001 & 0.142 & 0.001 & 0.160 \\
\hline OPLEV & $-0.005 * * *$ & -3.741 & $-0.005 * * *$ & -3.453 & 0.037 & 0.443 & 0.022 & 0.262 & -0.006 & -1.102 & -0.006 & -1.087 \\
\hline$T R V O L$ & $0.008 * * *$ & 38.280 & $0.008 * * *$ & 37.763 & $0.084 * * *$ & 7.077 & $0.076^{* * *}$ & 6.273 & $-0.010 * * *$ & -11.288 & $-0.010 * * *$ & -11.066 \\
\hline$N \_A N A L Y S T$ & $-0.001 * * *$ & -3.195 & $-0.001 * * *$ & -3.190 & $0.097 * * *$ & 5.057 & $0.087 * * *$ & 4.548 & $-0.003 * * *$ & -2.677 & $-0.003 * *$ & -2.355 \\
\hline$S \bar{P} 500$ & $-0.005 * * *$ & -10.717 & $-0.004 * * *$ & -10.298 & 0.027 & 0.956 & 0.008 & 0.283 & $-0.008 * * *$ & -5.353 & $-0.008 * * *$ & -5.327 \\
\hline$S T D V \_R O A$ & $0.031 * * *$ & 19.485 & $0.029 * * *$ & 17.242 & 0.006 & 0.085 & 0.017 & 0.218 & $0.048 * * *$ & 4.021 & $0.046^{* * *}$ & 3.650 \\
\hline HERFIN & & & $0.001 * *$ & 2.029 & & & -0.044 & -1.325 & & & $0.007 * *$ & 2.529 \\
\hline NFIRM & & & $0.007 * * *$ & 8.930 & & & $-0.257 * * *$ & -4.939 & & & $0.019 * * *$ & 3.322 \\
\hline ROACORR & & & $-0.001 *$ & -1.861 & & & $0.161 * * *$ & 7.607 & & & 0.001 & 0.764 \\
\hline Constant & $-0.025 * * *$ & -7.466 & $-0.058 * * *$ & -9.109 & $-3.662 * * *$ & -11.853 & $-2.345 * * *$ & -4.866 & $0.425 * * *$ & 27.731 & $0.311 * * *$ & 8.339 \\
\hline Year FE & Yes & & Yes & & Yes & & Yes & & Yes & & Yes & \\
\hline Industry FE & Yes & & Yes & & Yes & & Yes & & Yes & & Yes & \\
\hline
\end{tabular}

Sample includes firm-year observations with at least three unique analyst forecasts and non-zero financial leverage from 1989 to 2017. The variable of interest, FINLEV, is defined as the ratio of financial debt $(D L C+D L T T)$ to total assets $(A T)$. All other variables in the table are defined in Appendix A. Industry dummies capture industry fixed effects with two-digit SIC code classifications. Standard errors are robust and clustered by firm ( $g v k e y)$. Continuous variables are winsorized at top and bottom 1 percent. $* * *, * *$, and * respectively denote significance at $1 \%, 5 \%$, and $10 \%$. 
TABLE 1.8 (a)

First-stage Regression Estimates to Calculate Predicted Leverage

\begin{tabular}{lcc}
\hline VARIABLES & Coefficient & t-stat \\
\hline & & \\
SIZE & $0.005^{* * *}$ & 9.32 \\
$R O A$ & $-0.347^{* * *}$ & -13.14 \\
$M B$ & $0.005^{* * *}$ & 6.44 \\
DEPRECIATION & $-0.478^{* * *}$ & -6.69 \\
TANGIBILITY & $0.105^{* * *}$ & 7.31 \\
$R N D$ & $-0.607^{* * *}$ & -18.45 \\
$R N D \_D U M$ & 0.001 & 0.034 \\
MED_DEBT & $0.495^{* * *}$ & 17.55 \\
Constant & $0.109^{* * *}$ & 9.23 \\
& & \\
Observations & 21,058 & \\
R-squared & 0.267 & \\
\hline
\end{tabular}


TABLE 1.8 (b)

Regression Analyses of Forecast Accuracy and Forecast Dispersion on Predicted Leverage

\begin{tabular}{|c|c|c|c|c|c|c|c|c|}
\hline \multirow[b]{2}{*}{ VARIABLES } & \multicolumn{4}{|c|}{ Dep Var: FORECAST ACCURACY } & \multicolumn{4}{|c|}{ Dep Var: FORECAST DISPERSION } \\
\hline & Coefficient & $t$-stat & Coefficients & $t$-stat & Coefficients & $t$-stat & Coefficients & $t$-stat \\
\hline$E(L E V)$ & $-2.063 * * *$ & -9.387 & $-1.779 * * *$ & -7.831 & $1.274 * * *$ & 7.912 & $1.123 * * *$ & 6.631 \\
\hline SIZE & $0.332 * * *$ & 31.093 & $0.343 * * *$ & 30.568 & $-0.124 * * *$ & -18.791 & $-0.132 * * *$ & -18.841 \\
\hline$M B$ & $0.036 * * *$ & 11.655 & $0.039 * * *$ & 12.230 & $-0.013 * * *$ & -7.435 & $-0.014 * * *$ & -7.540 \\
\hline HORIZON & -0.034 & -1.399 & -0.040 & -1.617 & $-0.033 * *$ & -2.001 & $-0.028 *$ & -1.690 \\
\hline$C h \_E P S$ & $-0.206^{*}$ & -1.691 & $-0.219 *$ & -1.804 & $1.100 * * *$ & 7.018 & $0.991 * * *$ & 6.384 \\
\hline LOSS & $-0.886 * * *$ & -31.188 & $-0.871 * * *$ & -29.820 & $0.798 * * *$ & 26.079 & $0.753 * * *$ & 24.755 \\
\hline$S T D V \_R O A$ & $-0.475 * * *$ & -5.273 & $-0.539 * * *$ & -4.113 & 0.102 & 1.326 & $0.419 * * *$ & 3.575 \\
\hline$N \_A N A L Y S T$ & $-0.165 * * *$ & -7.208 & $-0.150 * * *$ & -6.609 & $0.113 * * *$ & 7.389 & $0.105 * * *$ & 6.865 \\
\hline$\overline{O P} L L E V$ & & & $-1.104 * * *$ & -10.764 & & & $0.480 * * *$ & 6.388 \\
\hline TANGIBILITY & & & $-0.164 * *$ & -2.019 & & & 0.066 & 1.157 \\
\hline$B I G 4$ & & & 0.041 & 1.425 & & & -0.005 & -0.312 \\
\hline DIVPAYER & & & 0.025 & 0.878 & & & -0.011 & -0.668 \\
\hline$S T D \_C F O$ & & & -0.139 & -0.828 & & & $-0.462 * * *$ & -3.135 \\
\hline$A C \overline{C R} U A L S$ & & & -0.190 & -1.566 & & & -0.085 & -0.792 \\
\hline & $4.757 * * *$ & 29.621 & $4.980 * * *$ & & $0.949 * * *$ & 10.018 & $0.912 * * *$ & 7.802 \\
\hline Year FE & Yes & & Yes & & Yes & & Yes & \\
\hline Industry FE & Yes & & Yes & & Yes & & Yes & \\
\hline Observations & 21,058 & & 20,461 & & 21,058 & & 20,461 & \\
\hline R-squared & 0.327 & & 0.334 & & 0.242 & & 0.243 & \\
\hline
\end{tabular}

Sample includes firm-year observations with at least three unique analyst forecasts from 1989 to 2017. The variable of interest, $E(L E V)$, is the predicted leverage calculated from a first-stage model. All other variables in the table are defined in Appendix A. Industry dummies capture industry fixed effects with two-digit SIC code classifications. Standard errors are robust and clustered by firm (gvkey). Continuous variables are winsorized at top and bottom 1 percent. $* * *, * *$, and $*$ respectively denote significance at $1 \%, 5 \%$, and $10 \%$. 
TABLE 1.8 (c)

Regression Analyses of Firm-specific Information Environment on Predicted Leverage

\begin{tabular}{|c|c|c|c|c|c|c|c|c|c|c|c|c|}
\hline \multirow[b]{2}{*}{ VARIABLES } & \multicolumn{4}{|c|}{ Dep Var: IDVOL } & \multicolumn{4}{|c|}{ Dep Var: SYNCH } & \multicolumn{4}{|c|}{ Dep Var: PIN } \\
\hline & Coeff & $t$-stat & Coeff & $t$-stat & Coeff & $t$-stat & Coeff & $t$-stat & Coeff & $t$-stat & Coeff & $t$-stat \\
\hline FINLEV & 0.004 & 1.303 & 0.005 & 1.588 & $-0.294^{*}$ & -1.803 & $-0.392 * *$ & -2.316 & 0.010 & 0.736 & 0.019 & 1.310 \\
\hline OPLEV & $-0.004 * * *$ & -3.078 & $-0.004 * * *$ & -2.940 & 0.026 & 0.328 & 0.031 & 0.387 & -0.005 & -0.867 & -0.006 & -0.979 \\
\hline TRVOL & $0.008 * * *$ & 37.819 & $0.008 * * *$ & 37.228 & $0.093 * * *$ & 8.204 & $0.086 * * *$ & 7.423 & $-0.009 * * *$ & -10.535 & $-0.009 * * *$ & -10.326 \\
\hline N_ANALYST & $-0.001 * * *$ & -3.131 & $-0.001 * * *$ & -3.178 & $0.107 * * *$ & 5.418 & $0.100 * * *$ & 5.051 & $-0.003 * * *$ & -2.958 & $-0.003 * * *$ & -2.643 \\
\hline$S \bar{P} 500$ & $-0.005 * * *$ & -10.921 & $-0.005^{* * *}$ & -10.307 & 0.020 & 0.692 & 0.001 & 0.052 & $-0.008 * * *$ & -4.914 & $-0.008 * * *$ & -4.965 \\
\hline$S T D V \_R O A$ & $0.031 * * *$ & 22.048 & $0.029 * * *$ & 19.832 & -0.094 & -1.486 & -0.082 & -1.193 & $0.047 * * *$ & 3.554 & $0.051 * * *$ & 3.455 \\
\hline HERFIN & & & $0.001 * *$ & 2.421 & & & -0.022 & -0.680 & & & $0.009 * * *$ & 2.930 \\
\hline NFIRM & & & $0.008 * * *$ & 9.231 & & & $-0.209 * * *$ & -3.913 & & & $0.023 * * *$ & 3.735 \\
\hline ROACORR & & & $-0.001 * *$ & -2.166 & & & $0.152 * * *$ & 7.174 & & & 0.001 & 0.431 \\
\hline Constant & $-0.031 * * *$ & -10.610 & $-0.077 * * *$ & -11.362 & $-4.207 * * *$ & -24.012 & $-2.953 * * *$ & -7.110 & $0.417 * * *$ & 33.166 & $0.244 * * *$ & 5.295 \\
\hline Year FE & Yes & & Yes & & Yes & & Yes & & Yes & & Yes & \\
\hline Industry FE & Yes & & Yes & & Yes & & Yes & & Yes & & Yes & \\
\hline
\end{tabular}

Sample includes firm-year observations with at least three unique analyst forecasts from 1989 to 2017 . The variable of interest, $E(L E V)$, is the predicted leverage calculated from a first-stage model. All other variables in the table are defined in Appendix A. Industry dummies capture industry fixed effects with two-digit SIC code classifications. Standard errors are robust and clustered by firm (gvkey). Continuous variables are winsorized at top and bottom 1 percent. $* * *, * *$, and $*$ respectively denote significance at $1 \%, 5 \%$, and $10 \%$. 
TABLE 1.9 (a)

Regression Analyses of Forecast Accuracy and Forecast Dispersion on Bank vs. Non-bank Debt

\begin{tabular}{|c|c|c|c|c|c|c|c|c|}
\hline \multirow[b]{2}{*}{ VARIABLES } & \multicolumn{4}{|c|}{ Dep Var: FORECAST ACCURACY } & \multicolumn{4}{|c|}{ Dep Var: FORECAST DISPERSION } \\
\hline & Coefficient & $t$-stat & Coefficients & $t$-stat & Coefficients & $t$-stat & Coefficients & $t$-stat \\
\hline$B A N K D E B T$ & $-0.320 * *$ & -2.122 & $-0.650 * * *$ & -4.423 & $0.515 * * *$ & 3.927 & $0.640 * * *$ & 4.876 \\
\hline NONBANKDEBT & $-0.869 * * *$ & -7.025 & $-1.113 * * *$ & -8.893 & $0.898 * * *$ & 8.070 & $0.968 * * *$ & 8.556 \\
\hline SIZE & $0.310 * * *$ & 20.166 & $0.320 * * *$ & 20.191 & $-0.128 * * *$ & -11.371 & $-0.137 * * *$ & -11.750 \\
\hline$M B$ & $0.032 * * *$ & 7.364 & $0.042 * * *$ & 9.221 & $-0.016^{* * *}$ & -6.017 & $-0.018 * * *$ & -6.857 \\
\hline HORIZON & $-0.075^{* *}$ & -2.238 & $-0.087 * * *$ & -2.608 & -0.025 & -0.987 & -0.016 & -0.607 \\
\hline$C h \_E P S$ & -0.018 & -0.120 & -0.112 & -0.761 & $0.799 * * *$ & 3.611 & $0.850 * * *$ & 3.830 \\
\hline$L O S S$ & $-0.837 * * *$ & -20.565 & $-0.837 * * *$ & -20.217 & $0.857 * * *$ & 16.481 & $0.840 * * *$ & 16.760 \\
\hline$S T D V \_R O A$ & $-1.021 * * *$ & -6.133 & $-1.037 * * *$ & -4.700 & $0.476 * *$ & 2.522 & $1.192 * * *$ & 3.983 \\
\hline$N \_A N A L Y S T$ & -0.041 & -1.236 & -0.045 & -1.402 & $0.066^{* * * *}$ & 2.586 & $0.068 * * *$ & 2.694 \\
\hline OPLLEV & & & $-1.513 * * *$ & -9.445 & & & $0.772 * * *$ & 5.968 \\
\hline TANGIBILITY & & & $-0.326 * * *$ & -2.939 & & & $0.233 * *$ & 2.445 \\
\hline BIG4 & & & 0.046 & 1.189 & & & -0.031 & -1.131 \\
\hline DIVPAYER & & & $0.071 *$ & 1.890 & & & $-0.044^{*}$ & -1.659 \\
\hline$S T D \_C F O$ & & & -0.347 & -1.099 & & & $-1.170 * * *$ & -3.547 \\
\hline$A C \overline{C R} U A L S$ & & & $-0.782 * * *$ & -4.018 & & & 0.231 & 0.947 \\
\hline Constant & $4.490 * * *$ & 19.234 & $4.618 * * *$ & 18.605 & $0.367 * *$ & 2.264 & $0.565 * * *$ & 3.098 \\
\hline Year FE & Yes & & Yes & & Yes & & Yes & \\
\hline Industry FE & Yes & & Yes & & Yes & & Yes & \\
\hline Observations & 9,178 & & 9,172 & & 9,178 & & 9,172 & \\
\hline R-squared & 0.374 & & 0.387 & & 0.279 & & 0.288 & \\
\hline
\end{tabular}

Sample includes firm-year observations with at least three unique analyst forecasts from 1989 to 2017 . The variables of interest, BANKDEBT and NONBANKDEBT, are respectively defined as the ratio of bank borrowing over total assets and the ratio of non-bank borrowing over total assets. All other variables in the table are defined in Appendix A. Industry dummies capture industry fixed effects with two-digit SIC code classifications. Standard errors are robust and clustered by firm (gvkey). Continuous variables are winsorized at top and bottom 1 percent. ***, **, and * respectively denote significance at $1 \%, 5 \%$, and $10 \%$. 
TABLE 1.9 (b)

Regression Analyses of Firm-specific Information Environment on Bank vs. Non-bank Debt

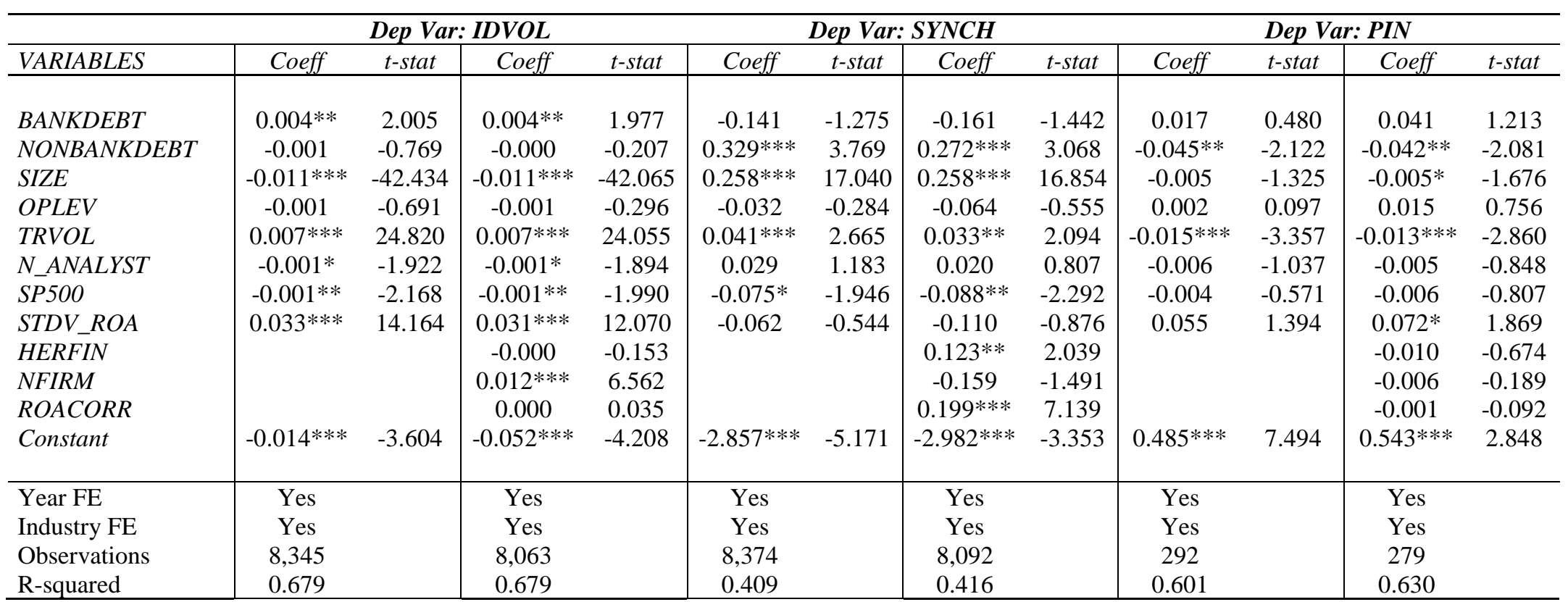

Sample includes firm-year observations with at least three unique analyst forecasts from 1989 to 2017. The variables of interest, BANKDEBT and NONBANKDEBT, are respectively defined as the ratio of bank borrowing over total assets and the ratio of non-bank borrowing over total assets. All other variables in the table are defined in Appendix A. Industry dummies capture industry fixed effects with two-digit SIC code classifications. Standard errors are robust and clustered by firm (gvkey). Continuous variables are winsorized at top and bottom 1 percent. ***, **, and * respectively denote significance at $1 \%, 5 \%$, and $10 \%$. 
TABLE 1.10

Regression Analyses of Forecast Accuracy and Forecast Dispersion on Financial Leverage

Panel A: Rated vs. Unrated

\begin{tabular}{|c|c|c|c|c|c|c|c|c|}
\hline \multirow[b]{3}{*}{ VARIABLES } & \multicolumn{4}{|c|}{ Dep Var: FORECAST ACCURACY } & \multicolumn{4}{|c|}{ Dep Var: FORECAST DISPERSION } \\
\hline & \multicolumn{2}{|c|}{ Rated } & \multicolumn{2}{|c|}{ Unrated } & \multicolumn{2}{|c|}{ Rated } & \multicolumn{2}{|c|}{ Unrated } \\
\hline & Coefficient & $t$-stat & Coefficients & $t$-stat & Coefficients & $t$-stat & Coefficients & $t$-stat \\
\hline FINLEV & $-0.677 * * *$ & -9.501 & $-0.600 * * *$ & -6.952 & $0.560 * * *$ & 10.710 & $0.454 * * *$ & 7.218 \\
\hline$S I Z E$ & $0.405^{* * *} *$ & 39.597 & $0.329 * * *$ & 33.259 & $-0.155^{* * *}$ & -20.691 & $-0.129 * * *$ & -17.868 \\
\hline$M B$ & $0.027 * * *$ & 9.237 & $0.027 * * *$ & 7.747 & $-0.011 * * *$ & -4.865 & $-0.010 * * *$ & -3.743 \\
\hline HORIZON & $-0.091 * * *$ & -3.214 & 0.001 & 0.044 & -0.025 & -1.180 & -0.035 & -1.486 \\
\hline$C h \_E P S$ & 0.097 & 0.650 & -0.154 & -1.096 & $1.047 * * *$ & 9.590 & $0.779 * * *$ & 7.632 \\
\hline$L O S S$ & $-0.861 * * *$ & -29.084 & $-0.813 * * *$ & -23.884 & $0.696 * * *$ & 32.061 & $0.871 * * *$ & 35.156 \\
\hline$S T D V \_R O A$ & $-0.596 * * *$ & -7.665 & $-1.033 * * *$ & -6.078 & $0.273 * * *$ & 4.773 & $0.565 * * *$ & 4.570 \\
\hline$N \_A N A L Y S T$ & $-0.090 * * *$ & -3.402 & $-0.184 * * *$ & -7.494 & $0.127 * * *$ & 6.548 & $0.087 * * *$ & 4.882 \\
\hline Constant & $3.610 * * *$ & 14.651 & $4.414 * * *$ & 15.864 & $1.101 * * *$ & 6.088 & $1.368 * * *$ & 6.756 \\
\hline Year FE & Yes & & Yes & & Yes & & Yes & \\
\hline Industry FE & Yes & & Yes & & Yes & & Yes & \\
\hline Observations & 12,103 & & 11,481 & & 12,103 & & 11,481 & \\
\hline R-squared & 0.344 & & 0.348 & & 0.233 & & 0.280 & \\
\hline
\end{tabular}


Panel B: High vs. Low Analysts Following

\begin{tabular}{|c|c|c|c|c|c|c|c|c|}
\hline & \multicolumn{4}{|c|}{ Dep Var: FORECAST ACCURACY } & \multicolumn{4}{|c|}{ Dep Var: FORECAST DISPERSION } \\
\hline & High Fo & wing & Low Foll & ing & High Fol & ving & Low Foll & ving \\
\hline VARIABLES & Coefficient & $t$-stat & Coefficients & $t$-stat & Coefficients & $t$-stat & Coefficients & $t$-stat \\
\hline FINLEV & $-0.827 * * *$ & -13.276 & $-0.989 * * *$ & -12.213 & $0.694 * * *$ & 14.414 & $0.652 * * *$ & 12.084 \\
\hline$S I Z E$ & $0.383 * * *$ & 45.644 & $0.265 * * *$ & 28.006 & $-0.133 * * *$ & -20.559 & $-0.101 * * *$ & -15.955 \\
\hline$M B$ & $0.026 * * *$ & 8.815 & $0.038 * * *$ & 11.154 & $-0.010 * * *$ & -4.474 & $-0.016 * * *$ & -7.251 \\
\hline HORIZON & -0.031 & -1.107 & $-0.069 * *$ & -2.033 & -0.027 & -1.283 & $-0.046^{* *}$ & -2.008 \\
\hline$C h \_E P S$ & 0.043 & 0.321 & -0.126 & -0.785 & $0.723 * * *$ & 7.043 & $1.193 * * *$ & 11.167 \\
\hline$L O S S$ & $-0.900 * * *$ & -31.160 & $-0.814 * * *$ & -23.200 & $0.803 * * *$ & 35.943 & $0.765 * * *$ & 32.686 \\
\hline$S T D V \_R O A$ & $-0.617 * * *$ & -7.340 & $-0.787 * * *$ & -6.064 & $0.254 * * *$ & 3.912 & $0.331 * * *$ & 3.826 \\
\hline N_ANALYST & 0.040 & 0.785 & $-0.246 * * *$ & -6.741 & $0.078 * *$ & 1.983 & $0.121 * * *$ & 4.968 \\
\hline Constant & $3.618 * * *$ & 14.972 & $5.205 * * *$ & 16.884 & $1.115 * * *$ & 5.967 & $0.978 * * *$ & 4.756 \\
\hline Year FE & Yes & & Yes & & Yes & & Yes & \\
\hline Industry FE & Yes & & Yes & & Yes & & Yes & \\
\hline Observations & 13,428 & & 10,156 & & 13,428 & & 10,156 & \\
\hline R-squared & 0.355 & & 0.315 & & 0.241 & & 0.273 & \\
\hline
\end{tabular}


Panel C: High vs. Low Institutional Shareholdings

\begin{tabular}{|c|c|c|c|c|c|c|c|c|}
\hline & \multicolumn{4}{|c|}{ Dep Var: FORECAST ACCURACY } & \multicolumn{4}{|c|}{ Dep Var: FORECAST DISPERSION } \\
\hline & \multicolumn{2}{|c|}{ High Inst. } & \multicolumn{2}{|c|}{ Low Inst. } & \multicolumn{2}{|c|}{ High Inst. } & \multicolumn{2}{|c|}{ Low Inst. } \\
\hline VARIABLES & Coefficient & $t$-stat & Coefficients & $t$-stat & Coefficients & $t$-stat & Coefficients & $t$-stat \\
\hline FINLEV & $-1.013 * * *$ & -14.185 & $-0.733 * * *$ & -10.731 & $0.829 * * *$ & 15.130 & $0.514 * * *$ & 11.087 \\
\hline SIZE & $0.333 * * *$ & 38.040 & $0.312 * * *$ & 33.390 & $-0.131 * * *$ & -19.400 & $-0.096 * * *$ & -15.076 \\
\hline$M B$ & $0.032 * * *$ & 9.579 & $0.034 * * *$ & 11.513 & $-0.012 * * *$ & -4.780 & $-0.013 * * *$ & -6.675 \\
\hline HORIZON & $-0.094 * * *$ & -2.836 & -0.014 & -0.505 & -0.023 & -0.906 & $-0.036^{*}$ & -1.922 \\
\hline$C h \_E P S$ & -0.024 & -0.163 & -0.031 & -0.218 & $1.009 * * *$ & 9.083 & $0.787 * * *$ & 8.024 \\
\hline LOSS & $-0.948 * * *$ & -29.821 & $-0.755 * * *$ & -24.169 & $0.853 * * *$ & 34.951 & $0.688 * * *$ & 32.467 \\
\hline$S T D V \_R O A$ & $-0.455 * * *$ & -5.035 & $-0.919 * * *$ & -7.840 & 0.095 & 1.362 & $0.478 * * *$ & 6.004 \\
\hline$N \_A N A L Y S T$ & $-0.265 * * *$ & -9.532 & $-0.096 * * *$ & -4.152 & $0.142 * * *$ & 6.662 & $0.095 * * *$ & 6.022 \\
\hline Constant & $4.680 * * *$ & 18.810 & $4.327 * * *$ & 13.325 & $0.881 * * *$ & 4.614 & $1.195 * * *$ & 5.419 \\
\hline Year FE & Yes & & Yes & & Yes & & Yes & \\
\hline Industry FE & Yes & & Yes & & Yes & & Yes & \\
\hline Observations & 11,792 & & 11,792 & & 11,792 & & 11,792 & \\
\hline R-squared & 0.351 & & 0.302 & & 0.263 & & 0.237 & \\
\hline
\end{tabular}


Panel D: Big vs. Small Firm

\begin{tabular}{|c|c|c|c|c|c|c|c|c|}
\hline & \multicolumn{4}{|c|}{ Dep Var: FORECAST ACCURACY } & \multicolumn{4}{|c|}{ Dep Var: FORECAST DISPERSION } \\
\hline & \multicolumn{2}{|c|}{ Big } & \multicolumn{2}{|c|}{ Small } & \multicolumn{2}{|c|}{ Big } & \multicolumn{2}{|c|}{ Small } \\
\hline VARIABLES & Coefficient & $t$-stat & Coefficients & $t$-stat & Coefficients & t-stat & Coefficients & t-stat \\
\hline FINLEV & $-0.772 * * *$ & -11.328 & $-0.655 * * *$ & -8.398 & $0.607 * * *$ & 11.412 & $0.473 * * *$ & 8.916 \\
\hline SIZE & $0.510 * * *$ & 41.536 & $0.318 * * *$ & 31.098 & $-0.232 * * *$ & -24.201 & $-0.123 * * *$ & -17.767 \\
\hline$M B$ & $0.018 * * *$ & 5.674 & $0.032 * * *$ & 9.695 & $-0.008 * * *$ & -3.107 & $-0.008 * * *$ & -3.528 \\
\hline HORIZON & $-0.116^{* * *}$ & -3.904 & 0.010 & 0.330 & -0.012 & -0.497 & $-0.037 *$ & -1.768 \\
\hline$C h \_E P S$ & -0.004 & -0.030 & -0.117 & -0.810 & $1.089 * * *$ & 9.802 & $0.745 * * *$ & 7.587 \\
\hline$L \overline{O S S}$ & $-0.856^{* * *}$ & -29.144 & $-0.765 * * *$ & -22.254 & $0.703 * * *$ & 30.688 & $0.848 * * *$ & 36.247 \\
\hline$S T D V \_R O A$ & $-0.563 * * *$ & -7.141 & $-1.189 * * *$ & -7.266 & $0.299 * * *$ & 4.860 & $0.524 * * *$ & 4.711 \\
\hline$N \_A N \bar{A} L Y S T$ & $-0.059 * *$ & -2.009 & $-0.152 * * *$ & -6.705 & $0.138 * * *$ & 6.012 & $0.069 * * *$ & 4.453 \\
\hline Constant & $3.177 * * *$ & 12.592 & $4.182 * * *$ & 15.073 & $1.500 * * *$ & 7.626 & $1.384 * * *$ & 7.335 \\
\hline Year FE & Yes & & Yes & & Yes & & Yes & \\
\hline Industry FE & Yes & & Yes & & Yes & & Yes & \\
\hline Observations & 11,792 & & 11,792 & & 11,792 & & 11,792 & \\
\hline R-squared & 0.346 & & 0.319 & & 0.249 & & 0.273 & \\
\hline
\end{tabular}




\section{APPENDIX A}

Variable Definition:

ACCURACY: negative one times log of absolute value difference between actual EPS and median forecasted EPS, scaled by year-end stock price.

DISPERSION: $\quad$ standard deviation of analyst forecast scaled by year-end stock price (times 100).

IDVOL: probability of informed trading collected from Soren Hvidkjaer's website.

SYNCH: standard deviation of excess return calculated from weekly return regression.

PIN: log-transformed r-square of weekly return regressions.

SIZE: $\quad \log$ of market value of equity of firm $i$ in year $t$.

$M B$ : ratio market value of equity to book value of equity in year $t$.

HORIZON: $\log$ of number of days between the forecast date and the earnings announcement date.

Ch_EPS: $\quad$ change in earnings-per-share, scaled by share price at the beginning of year $t$.

LOSS: indicator variable 1 , if negative earnings is reported in year $t, 0$ otherwise.

$S T D V_{-} R O A: \quad$ standard deviation of ROA (IB/AT) over last 5 years (with min 3 years of data).

$N \_A N A L Y S T: \quad \log$ of number of analysts following firm $i$ in year $t$.

INST_HOLDING: average percentage of shares owned by institutional investors throughout the year $t$.

OPLLEV: operating liability leverage, scaled by total assets, both at the end of year $t$.

TANGIBILITY: net property plant and equipment, scaled by total assets, both at the end of year $t-1$.

BIG4: indicator variable 1 , if firm $i$ is audited by a Big4 auditor in year $t$.

DIVPAYER: indicator variable 1, if common stock dividend is paid in year t, 0 otherwise.

STD_CFO: standard deviation of operating cash flows over last 5 years (min 3 years of data).

ACCRUALS: net income minus cash flow from operations in year $t$ scaled by total assets in $t-1$.

DEPREICATION: depreciation scaled by total assets in $t-1$.

$R N D$ : research and development expenses scaled by total assets in $t-1$.

RND_DUM: indicator variable 1 , if research and development expense is missing.

$M E D \_D E B T:$ industry media debt ratio for year $t-1$.

TRVOL: average daily stock turnover of firm $i$ in year $t$.

SP500: indicator variable 1 for S\&P 500 firms.

HERFIN: Herfindahl index for industry concentration based on sales.

NFIRM: number of firms in each Fama-French 48 industry.

ROACORR: correlation between industry-average ROA and firm's ROA over past 12 quarters. 


\section{ESSAY 2: THE EFFECTS OF OPERATING LIABILITY LEVERGE ON A FIRM'S INFORMATION ENVIRONMENT}

\section{INTRODUCTION}

This paper investigates whether different types of liabilities in capital structure of a firm generates additional information for market participants and improves information environment of a firm. A firm can finance its assets through financial debt and through operating liabilities. ${ }^{6}$ Studies in accounting and finance show that leverage arising from operating activities and financing activities have differential effects on firm profitability and firm value (Nissim and Penman 2003), transaction costs (Ferris 1981), and informational role and monitoring costs (Smith 1987; Biais and Gollier 1997; Petersen and Rajan 1997). Operating liability leverage constitutes almost one-third of the total assets reported in the balance sheets by publicly traded firms in the USA (Welch 2011). In this paper, I examine differential effect of financial leverage and operating liability leverage on firm information environment.

Capital structure theories suggest that debt generates additional information, works as a monitoring device and hence improves overall information environment of a firm (e.g., Harris and Raviv 1990; Jensen 1986). Finance and accounting literature also indicate that debt accrues additional costs to the firm (Harris and Raviv 1991; Bolton and Scharfstein, 1990; Parsons and Titman, 2008), reduces financial flexibility (Harris and Raviv 1990; Zingales 1998), and increases agency conflicts (Jensen and Meckling 1976; Myers 1977),

\footnotetext{
${ }^{6}$ Financing leverage represents borrowings from banks, financial institutions, or bondholders while operating liability leverage relates to borrowings from suppliers, customers, and employees including trade and taxes payable, deferred revenues, and pension liabilities.
} 
which may have negative effects on the information environment of the firm. However, in a firm's balance sheet there are two different types of liabilities that can arise from the two components of leverage — operating liability leverage and financing leverage. For example, financing leverage is traded in well-functioning capital market, while operating liability leverage is traded in "less than perfect" input and output markets (Nissim and Penman 2003). Moreover, operating liabilities also include some accrual components that are susceptible to opportunistic managerial behavior, ${ }^{7}$ while financial liabilities are contractual obligations that are measurable with higher degree of reliability (Richardson, Sloan, Soliman and Tuna 2005). Thus, my first hypothesis relates to the differential effects of operating liability leverage and financing leverage on for, information environment.

I then examine different components of operating liability leverage. Prior studies suggest some operating liability items (e.g., accounts payable, tax payable) are contractual and hence can be measured with greater degree of accuracy, while others (e.g., deferred revenue, pension obligation etc.) are susceptible to measurement errors or managerial discretion (Nissim and Penman 2003; Richardson et al. 2005). Accordingly, I examine whether the information quality of a firm is differentially associated with the two types of operating liability leverage by decomposing operating liability leverage into contractual versus estimated operating liability leverage. My second hypothesis is that estimated operating liability leverage will have a differential effect on a firm's information environment than contractual operating liability leverage.

\footnotetext{
7 For example, pension liabilities are obligations to employees but are estimated based on actuarial assumptions. Similarly, deferred revenues are obligations to customers, but involve managerial discretion to allocate revenues over different time periods.
} 
To proxy for information environment quality, I use analysts' forecast-based measures - accuracy and forecast dispersion, and firm-specific measures - idiosyncratic volatility, synchronicity and PIN. Based on prior studies, I argue that better information environment is associated with higher analysts' forecast accuracy and lower forecast dispersion, and higher firm specific information to the market participant. To test the hypotheses, I use a sample of 23,584 firm-year observations from 1989 to 2017. I find that both operating liability leverage and financing leverage is negatively associated with the information environment of a firm. The finding of a significant negative association between information environment and operating liability leverage is consistent with the conjecture that the liquidity risk and estimation risks associated with components of operating liability leverage increases managerial discretion over financial reporting and thus weakens the quality of information available to the market participants. Consistent with this argument, when I decompose operating liability leverage into estimated versus contractual operating liability leverage, I find that both are also negatively associated with the firm's information environment.

Overall, findings of this study suggest that additional information generated by financial and operating liability leverage does not necessarily contribute to the information environment of a firm, rather firms with no/low financial as well operating liabilities have incentives to provide superior information and facilitates market participants to make informed decisions. I contribute to the literature by providing evidence of effects of (a) operating liability leverage versus financial liability leverage, and (b) contractual versus estimated operating liability leverage, on the information environment of a firm. Thus, this study highlights the importance of distinguishing between different types of leverage in the 
studies related to capital market participants and their decision making. I also add to the literature on agency costs and the differential effects of various types of leverage in finance and accounting settings.

Section II reflects on prior studies and develops testable hypotheses. Section III presents variable measurements and research design, followed by a discussion on results from main analyses and additional tests in section IV and section V, respectively. Section VI concludes the study.

\section{RELATED LITERATURE AND HYPOTHESIS DEVELOPMENT}

\section{Operating Liability Leverage versus Financing Leverage}

Studies in accounting and finance view leverage as the use of financing liabilities (i.e., interest-bearing debt) in the capital structure. The lack of attention to leverage arising from "non-financing" liabilities is surprising, since operating liability leverage represents about one-third of the total assets (Welch 2011).

Nissim and Penman (2003) suggest that operating liability leverage and financing leverage are valued differently in the stock market. Since these two types of liabilities are traded in two different markets, reflect different contractual terms, and add value to the firm in different ways, I argue that operating liabilities generate different set of information in the market and are thus affect a firm's information environment differently than financial leverage.

Specifically, prior studies find that operating liabilities lever profitability more than financing liabilities (Nissim and Penman 2003) and facilitate aggregation of information and alleviate the information asymmetry problem (Biais and Gollier 1997). Further, 
operating liabilities have informational advantage over financing liabilities in evaluating the credit worthiness of clients, and in monitoring and requiring repayment of credit (Smith 1987; Mian and Smith 1992; Petersen and Rajan 1997). However, higher operating liabilities may also indicate difficulties making payments to suppliers and declining operating performance; Petersen and Rajan (1995) show that firm that are credit constrained use higher operating liabilities.

Further, financial reporting of operating liabilities involves estimates and managerial discretion (for example, such as those related to deferred revenue or pension liabilities), while financing liabilities represent contractual obligations that can be measured with higher degree of reliability (Richardson et al. 2005). Such higher discretion in measuring operating liability leverage can also increase management's propensity to misreport financial statements. Extending such logic, I argue that market participants receive different signals, directly or indirectly, from two different sets of leverage.

Finally, in the event of bankruptcy, operating liabilities usually receive precedence over financial liabilities. Welch (2011) notes that bankruptcy trustees often grant suppliers payments in order to help the company continue to be a going concern; similarly, employee compensation and benefits receive higher priority to retain key employees, and tax liabilities also are senior to major financial liabilities in bankruptcies.

In summary, the empirical evidence from prior studies suggests that the implications of operating liability leverage for the riskiness of operations and financial reporting of the client can be different from those of financial leverage. To the extent such differences in generating additional information manifest in the form information quality, my first hypothesis is: 
$H_{1}$ : The association with information environment is different for operating liability

leverage and financing leverage.

\section{Types of Operating Liability Leverage and Information Environment}

Prior research suggests that components of operating liabilities can differ in the degree of reliability (Nissim and Penman 2003; Richardson et al. 2005). Some of the operating liabilities, such as accounts payable and tax payable are measurable with a higher degree of reliability; I refer to such items as contractual operating liability. Some other operating liability items involve estimation and managerial discretion; I refer to such items, including deferred revenues, accrued expenses and post-retirement benefit obligations, as estimated operating liabilities. With the involvement of discretion in estimating the latter type of liabilities, management has a greater propensity to behave opportunistically. For example, management might choose to overstate (or understate) pension liabilities or defer (or expedite) more revenue than earned through services or sales (Nissim and Penman 2003). Similarly accrued expenses include many estimated items involving managerial discretion, such as warranty expenses, accrued restructuring charges and other estimated accrued expenses.

I argue that a firm's information environment is opaque in the presence of higher managerial discretion. As estimated operating liability leverage involves higher managerial discretion, it is more likely to be associated with lower quality of information compared to contractual operating liability leverage. This in turn will result in estimated operating liability leverage having a bigger effect, than contractual operating liability leverage, on information environment. Thus, my next hypothesis is: 
$\mathrm{H}_{2}$ : The association with information environment is higher for estimated operating

liability leverage than for contractual operating liability leverage.

\section{METHOD}

\section{Proxies of Information Environment:}

To proxy for information environment, I first use two measures based on analysts' earnings forecast -forecast accuracy (ACCURACY) and forecast dispersion (DISPERSION). Consistent with the previous essay, I rely on prior studies and draw that better information environment is associated with higher analysts' forecast precision and lower forecast dispersion. To examine the relation between operating liability leverage and information environment, I use the following model:

$$
\begin{gathered}
\text { ACCURACY }_{i, t} \text { or DISPERSION } \\
\text { IndustryFE }+ \text { YearFE }+\varepsilon
\end{gathered}
$$

I define forecast accuracy as the negative of absolute value difference between the actual EPS and the median analysts forecast scaled by year-end stock price. I define forecast dispersion as the standard deviation of analysts' forecasts (forecast dispersion) scaled by ending stock price. To control for firm fundamentals that potentially could drive both leverage and analysts' forecast, I include (1) log of market capitalization at the beginning of the year (SIZE), (2) market to book ratio $(M B)$, (3) changes in earnings per share over year $t\left(C h \_E P S\right),(4)$ an indicator variable for negative profit (LOSS), and (5) standard deviation of return-on-assets over last five years (STD_ROA). I also control for $\log$ of number of analysts $\left(N \_A N A L Y S T\right)$, and log of number of days between analysts forecast and earnings announcement (HORIZON). In an extended model, I further control 
for firm operating liability leverage (OPLLEV), asset tangibility (TANGIBILITY), audit quality $(B I G 4)$, dividend paying status (DIVPAYER), volatility of cash flow (STD_CFO), and total accruals $(A C C R U A L S)$.

\section{Firm-specific Measures of Information Environment}

To capture firm-specific information environment I use three measures idiosyncratic volatility, synchronicity, and probability of informed trading. Following Brockman and Yan (2009), my constructs for idiosyncratic volatility and synchronicity are derived from regressing weekly stock returns on contemporaneous and lagged weekly market returns, as well as on contemporaneous and lagged weekly industry returns. Idiosyncratic volatility is calculated as the standard deviation of regression residuals, whereas synchronicity is estimated from $\log$ transformation of unbounded $\mathrm{R}^{2}$. I use extended PIN measure suggested by Brown and Hillegeist $(2007)^{8}$. I calculate all three firm-specific measures, idiosyncratic volatility (IDVOL), synchronicity $(S Y N C H)$ and PIN for each firm-year and estimate the following model:

$$
\begin{aligned}
& \qquad I D V O L_{i, t} \text { or SYNCH } H_{i, t} \text { or PIN } N_{i, t}=\beta_{0}+\beta_{1} O P L L E V_{i, t}+\sum_{k} \beta_{k} \text { FirmControls }+ \\
& \text { IndustryFE }+ \text { YearFE }+\varepsilon
\end{aligned}
$$

To isolate the effects of debt on firm-specific information environment, I control for confounding factors suggested in previous studies (Piotroski and Roulstone, 2004; Brockman and Yan 2010). Specifically, I control for firm size (SIZE), average daily turnover $(T R V O L)$, standard deviation of return-on-assets (STDV_ROA), operating

\footnotetext{
${ }^{8}$ I use annual PIN data for period covering 1993 to 2010 available at Professor Stephen Brown's website (http://scholar.rhsmith.umd.edu/sbrown/pin-data).
} 
leverage (OPLLEV), analysts following ( $\left.N \_A N A L Y S T\right)$, and firm visibility (SP500). In an extended model, I further control for Herfindahl industry concentration index (HERFIN), number of firms in the industry group (NFIRM), and firm-industry earnings correlation (ROACORR).

\section{Measures of Operating Liability Leverage}

The independent variable of interest of this study is operating liability leverage (OPLLEV), measured as total liability minus financial liabilities scaled by total assets. To test my second hypothesis (H2), I decompose operating liability leverage into contractual operating liability leverage (CONOPL) and estimated operating liability leverage (ESTOPL). I define estimated operating liability leverage as the sum of unearned revenue, accrued expenses and pension liabilities, scaled by total assets; contractual operating liability leverage is the ratio of all other liabilities to total assets.

\section{Sample and Data}

Data for this study is primarily obtained come from COMPUSTAT annual, CRSP, I/B/E/S and Thompson Reuters 13F Institutional Holdings database. Initial sample includes firm-year observations for the period beginning in 1989 and ending in 2017. Following prior studies, I exclude firms incorporated outside USA and firms in the financial (SIC 6000-6999) and utility (SIC 4900-4999) sector. To avoid potential data error and outliers, I exclude observations with less than one million in market capitalization, market price less than a-dollar and observations with leverage less than zero or greater than one (Flannery and Rangan 2006; Warr et al. 2012). To ensure sufficient observations for the estimation of analysts' forecast accuracy and dispersion, I require firms to be followed by at least three 
analysts. After deleting observations with missing values for variables in the baseline model, I winsorize each continuous variable at both top and bottom one-percentile. Final sample for this study consists of 23,584 firm-year observations for 4,419 unique firms.

\section{RESULTS}

\section{Descriptive Statistics}

Table 2.1 presents statistics for key variables used in this study. Firms finance 22\% of their total assets by financial debt (FINLEV), and $28 \%$ by non-financial liabilities (OPLLEV). Most of the operating liability leverage (22\%) is contractual like accounts payable, taxes payable, where as only $6 \%$ is estimated. The mean (median) size of firms is $\$ 7,269$ (\$1,310) million and the mean (median) market-to-book ratio is 3.53 (2.42).

Table 2.2 reports Pearson pairwise correlation coefficients. Correlation coefficients indicate that operating leverage is positively correlated with analysts' forecast accuracy and forecast dispersion. Table 2 also indicate that the correlation coefficients are not significantly high enough to create multi-collinearity problem in regression estimations. ${ }^{9}$

\section{Regression Analyses - Forecast Accuracy and Dispersion:}

Table 2.3(a) and 2.3(b) presents the regression estimates respectively for analysts forecast-based measures and firm-specific measures. Table 2.3(a) reports that the coefficient estimate of TOTLEV is negative (positive) and significant (p-value <.001) with forecast accuracy (dispersion), indicating that a firm's information environment is decreasing with leverage. When I replace TOTLEV with financing leverage (FINLEV) and

\footnotetext{
${ }^{9}$ Variance inflation factors (VIF) in all regression specifications are less than 3.
} 
operating liability leverage (OPLLEV), results show similar pattern. The coefficient for FINLEV and OPLLEV is negative and significant (p-value <.001) for forecast accuracy, and positive and significant ( $\mathrm{p}$-value $<.001)$ forecast dispersion. However, the difference in coefficients between FINLEV and OPLLEV is not statistically significant, supporting the notion that increase in liability, irrespective of its type, weakens information environment of a firm. Table 2.3(b) reports results for firm-specific information environment. Consistent with results reported in table 2.3(a), regression estimates for idiosyncratic volatility $(I D V O L)$ is negative for total leverage, and financial and operating leverage. However, I do not find significantly strong association of leverage and its type with a firm's information environment.

\section{Contractual versus Estimated Operating Liability Leverage}

Table 2.4(a) and 2.4(b) replace operating liability leverage (OPLLEV) with two variables representing contractual operating liabilities (CONOPL) and estimated operating liabilities (ESTOPL). The coefficients of ESTOPL and CONOPL are negative and significant ( $\mathrm{p}$-value <.001) for analysts forecast accuracy, and positive and significant for forecast dispersion. A test of differences shows that the coefficient for ESTOPL is not significantly (p-value <.001) different from that for CONOPL. This finding supports the second hypothesis that estimated operating liabilities are associated with higher audit fees compared to contractual operating liabilities.

Next, I report regression estimates of firm-specific information environment on types of operating liability leverage in Table 2.4(b). Results for the idiosyncratic volatility $(I D V O L)$ are in the first four columns, results for synchronicity $(S Y N C H)$ in the middle four columns and results for probability of informed trading (PIN) are in the final four 
columns. The base model tests primary association between the dependent variable and the variable of interest, whereas an extended model is used to control for proxies for additional industry attributes. Regression results for idiosyncratic volatility (IDVOL) show that the coefficient for both estimated operating liability leverage (ESTOPL) and contractual operating liability leverage $(C O N O P L)$ are negative and statistically significant. These results are consistent with the results reported in table 2.4(a). However, coefficient estimates for synchronicity $(S Y N C H)$ and $P I N$ are respectively positive and negative but are not statistically significant.

\section{ADDITIONAL ANALYSES}

My primary findings indicate that operating liability leverage is negatively associated a firm's information environment. However, prior studies argue that a firm's information environment is also a function of other firm fundamentals, such as size, rating status, analysts following and institutional shareholdings. To test whether the relation between financial leverage and firm information environment are conditional on such factors, I split the sample based on these variables and rerun the regression model separately. Results are reported in Table 2.6.

First, I rerun regressions reported in the main analyses separately for two subsamples: rated firms and unrated firms. Results are reported in the Panel A of Table 2.6. In both-sub samples the coefficients of estimated and contractual liability leverage are negative (positive) and significant $(\mathrm{p}<.001)$ for analysts' forecast accuracy (dispersion). However, the coefficient estimate for unrated firms are stronger for contractual liability leverage than that for estimated liability leverage. Second, firms that are followed by fewer 
analysts are more likely to have weaker information environment. To test this prediction, I divide the sample into two sub-samples: low analysts following, and high analysts following and rerun the bassline regression. Results are reported in the first two columns of Panel B of Table 2.6. Consistent with previously documented results, the coefficients of ESTOPL and CONOPL are significant $(\mathrm{p}<.001)$ and negative (positive) for analysts forecast accuracy (dispersion). Results further indicate that when analysts monitoring is high, the role of estimated operating liability becomes less pronounced. Third, institutional shareholders also work as an alternative governance mechanism and generate additional information for market participants. To investigate the relative effects of institutional shareholdings, I estimate the baseline model on two subsamples: low institutional firms and high institutional firms. Results are reported in Panel C of Table 2.6. Consistent with prior findings, both ESTOPL and CONOPL are significant (p <.001) and negatively (positively) associated with analysts forecast precision (dispersion). Results further indicate that the effect is more pronounced when monitoring from external shareholders is low. Finally, I conduct separate regressions for sub-samples - large firms and small firms, where large (small) firms are those with total assets higher (lower) than the annual median. Panel D of Table 2.6 shows that the coefficient of ESTOPL and CONOPL is negative and significant $(\mathrm{p}<.001)$ for forecast accuracy, and positive and significant $(\mathrm{p}<.001)$ for forecast dispersion. However, the effects are higher for smaller firms than for larger firms, which supports the notion that smaller firms are characterized by weaker corporate governance, internal control systems and external monitoring. Studies also suggest that smaller firms are riskier than larger firms (e.g., Beaver, Kettler and Scholes 1970; Fama 
and French 1993). Thus, any change in information environment with respect to change in operating liabilities is stronger for smaller firms than for larger firms.

\section{CONCLUSION}

Studies in accounting and finance have long been considered leverage as a proxy of liquidity and bankruptcy risks, increased agency conflicts and risks for financial misstatements. In this paper, I show that, in contrast with prior arguments, the implications of leverage on a firm's information environment do not depend on whether the leverage originates from operating activities or financing activities. I first document a significant positive association between a firm's total leverage and its information environment. When I replace total leverage with operating liability leverage and financing leverage, I find the consistent association for both the types of leverage. This suggests that the risk of financial distress and financial misreporting as well as the agency cost of debt outweigh the benefits from monitoring and the disciplining effects of financing debt. In addition, I find that the effects of liquidity risk, as well as estimation risks associated with operating liability leverage weakens a firm's information environment. I further also examine the differential effects of estimated versus contractual operating liability leverage and consistently find that both contractual and estimated operating liabilities are negatively associated with the measures of information environment. 


\section{REFERENCES}

Biais, B. and Gollier, C., 1997. Trade credit and credit rationing. Review of financial studies 10(4): 903-937.

Ferris, J.S., 1981. A transactions theory of trade credit use. The Quarterly Journal of Economics: 243-270.

Harris, M. and Raviv, A., 1990. Capital structure and the informational role of debt. The Journal of Finance, 45(2): 321-349.

Harris, M. and Raviv, A., 1991. The theory of capital structure. the Journal of Finance, 46(1): 297-355.

Jensen, M.C. and Meckling, W.H., 1976. Theory of the firm: Managerial behavior, agency costs and ownership structure. Journal of financial economics, 3(4): 305-360.

Jensen, M.C., 1986. Agency cost of free cash flow, corporate finance, and takeovers. Corporate Finance, and Takeovers. American Economic Review, 76(2).

Myers, S.C., 1977. Determinants of corporate borrowing. Journal of financial economics, 5(2): 147-175.

Nissim, D. and Penman, S.H., 2003. Financial statement analysis of leverage and how it informs about profitability and price-to-book ratios. Review of Accounting Studies, 8(4): 531-560.

Petersen, M.A. and Rajan, R.G., 1995. The effect of credit market competition on lending relationships. The Quarterly Journal of Economics: 407-443.

Petersen, M.A. and Rajan, R.G., 1997. Trade credit: theories and evidence. Review of financial studies, 10(3): 661-691.

Richardson, S.A., Sloan, R.G., Soliman, M.T. and Tuna, I., 2005. Accrual reliability, earnings persistence and stock prices. Journal of accounting and economics, 39(3): 437-485.

Smith, J.K., 1987. Trade credit and informational asymmetry. The Journal of Finance, 42(4): 863-872.

Welch, I., 2011. Two Common Problems in Capital Structure Research: The FinancialDebt-To-Asset Ratio and Issuing Activity Versus Leverage Changes. International Review of Finance, 11(1): 1-17.

Zingales, L., 1998. Survival of the Fittest or the Fattest? Exit and Financing in the Trucking Industry. The Journal of Finance, 53(3): 905-938. 
Beaver, W., Kettler, P. and Scholes, M., 1970. The association between market determined and accounting determined risk measures. The Accounting Review, 45(4), pp.654682.

Bolton, P. and Scharfstein, D.S., 1990. A theory of predation based on agency problems in financial contracting. The American economic review, pp.93-106.

Brockman, P. and Yan, X.S., 2009. Block ownership and firm-specific information. Journal of Banking \& Finance, 33(2), pp.308-316.

Brown, S. and Hillegeist, S.A., 2007. How disclosure quality affects the level of information asymmetry. Review of Accounting Studies, 12(2-3), pp.443-477.

Fama, E.F. and French, K.R., 1993. Common risk factors in the returns on stocks and bonds. Journal of financial economics, 33(1), pp.3-56.

Parsons, C. and Titman, S., 2008. Handbook of Corporate Finance: Empirical Corporate Finance. Capital structure and corporate strategy.

Ross, S.A., 1977. The determination of financial structure: the incentive-signalling approach. The bell journal of economics, pp.23-40. 
TABLE 2.1

Descriptive Statistics

\begin{tabular}{|c|c|c|c|c|c|c|}
\hline Variables & Observations & Mean & S.D. & $1^{\text {st }}$ quartile & Median & $3^{\text {rd }}$ quartile \\
\hline$T L E V$ & 23,584 & 0.50 & 0.20 & 0.35 & 0.52 & 0.65 \\
\hline FINLEV & 23,584 & 0.22 & 0.18 & 0.06 & 0.21 & 0.34 \\
\hline OPLLEV & 23,584 & 0.28 & 0.12 & 0.19 & 0.26 & 0.35 \\
\hline ESTOPL & 23,584 & 0.06 & 0.08 & 0.00 & 0.04 & 0.09 \\
\hline CONOPL & 23,584 & 0.22 & 0.12 & 0.12 & 0.20 & 0.29 \\
\hline$A C C U R A C Y$ & 23,584 & 6.31 & 1.45 & 5.40 & 6.40 & 7.36 \\
\hline DISPERSION & 23,584 & 0.47 & 0.99 & 0.06 & 0.15 & 0.41 \\
\hline$I D V O L$ & 21,437 & 0.05 & 0.02 & 0.03 & 0.04 & 0.06 \\
\hline SYNCH & 21,502 & -0.64 & 1.10 & -1.37 & -0.63 & 0.11 \\
\hline PIN & 6,461 & 0.16 & 0.05 & 0.12 & 0.15 & 0.19 \\
\hline SIZE & 23,584 & 7.30 & 1.75 & 6.07 & 7.20 & 8.42 \\
\hline$M B$ & 23,584 & 3.53 & 3.83 & 1.53 & 2.42 & 3.97 \\
\hline HORIZON & 23,584 & 3.81 & 0.38 & 3.59 & 3.85 & 4.08 \\
\hline$C h \_E P S$ & 23,584 & 0.00 & 0.08 & -0.01 & 0.01 & 0.02 \\
\hline LOSS & 23,584 & 0.20 & 0.40 & 0.00 & 0.00 & 0.00 \\
\hline$S T D V \_R O A$ & 23,584 & 0.08 & 0.13 & 0.02 & 0.04 & 0.08 \\
\hline$N \_A N A L Y S T$ & 23,584 & 2.02 & 0.54 & 1.61 & 1.95 & 2.40 \\
\hline INST_HOLDING & 23,584 & 0.65 & 0.23 & 0.50 & 0.68 & 0.84 \\
\hline TANGIBILITY & 23,548 & 0.31 & 0.24 & 0.12 & 0.25 & 0.46 \\
\hline BIG4 & 23,584 & 0.74 & 0.44 & 0.00 & 1.00 & 1.00 \\
\hline DIVPAYER & 23,584 & 0.48 & 0.50 & 0.00 & 0.00 & 1.00 \\
\hline$S T D \_C F O$ & 22,927 & 0.08 & 0.10 & 0.03 & 0.05 & 0.09 \\
\hline ACCRUALS & 23,584 & -0.07 & 0.09 & -0.10 & -0.06 & -0.02 \\
\hline TRVOL & 23,584 & 18.49 & 1.60 & 17.36 & 18.49 & 19.59 \\
\hline SP500 & 23,584 & 0.30 & 0.46 & 0.00 & 0.00 & 1.00 \\
\hline HERFIN & 23,584 & 6.54 & 0.56 & 6.16 & 6.45 & 6.83 \\
\hline NFIRM & 23,584 & 5.35 & 0.91 & 4.76 & 5.47 & 5.96 \\
\hline ROACORR & 22,668 & 0.22 & 0.40 & -0.08 & 0.23 & 0.54 \\
\hline
\end{tabular}


TABLE 2.2

\section{Correlation Matrix}

\begin{tabular}{|c|c|c|c|c|c|c|c|c|c|c|}
\hline & $T L E V$ & FINLEV & OPLLEV & ESTOPL & CONOPL & ACCURACY & DISPERSION & $I D V L O$ & SYNCH & PIN \\
\hline$T L E V$ & 1 & & & & & & & & & \\
\hline FINLEV & 0.7354 & 1 & & & & & & & & \\
\hline OPLLEV & 0.4473 & -0.2745 & 1 & & & & & & & \\
\hline ESTOPL & 0.0572 & -0.0181 & 0.1041 & 1 & & & & & & \\
\hline CONOPL & 0.4195 & -0.2623 & 0.946 & -0.2173 & 1 & & & & & \\
\hline$A C C U R A C Y$ & -0.1441 & -0.1863 & 0.0414 & 0.0832 & 0.0138 & 1 & & & & \\
\hline DISPERSION & 0.1554 & 0.163 & 0.0021 & -0.0539 & 0.0201 & -0.4459 & 1 & & & \\
\hline$I D V O L$ & -0.0444 & 0.1033 & -0.2055 & 0.2746 & -0.2929 & -0.2324 & 0.2197 & 1 & & \\
\hline SYNCH & 0.033 & -0.0232 & 0.0819 & 0.0411 & 0.0704 & 0.1062 & -0.0707 & -0.3652 & 1 & \\
\hline$P I N$ & -0.1263 & 0.019 & -0.2069 & -0.2742 & -0.1178 & -0.2566 & 0.153 & 0.1962 & -0.3015 & 1 \\
\hline SIZE & 0.1039 & -0.101 & 0.2858 & 0.1076 & 0.2501 & 0.3987 & -0.2742 & -0.4453 & 0.4317 & -0.6475 \\
\hline$M B$ & 0.0495 & -0.0086 & 0.0767 & 0.1544 & 0.0223 & 0.2081 & -0.1387 & 0.0357 & 0.0586 & -0.1544 \\
\hline HORIZON & -0.0729 & -0.0985 & 0.0257 & -0.0735 & 0.0487 & 0.0099 & -0.0133 & -0.0544 & -0.0094 & 0.0557 \\
\hline$C h \_E P S$ & 0.0003 & -0.0095 & 0.0123 & 0.0036 & 0.0112 & 0.0044 & 0.0685 & 0.0437 & -0.0288 & 0.0265 \\
\hline$L O S S$ & 0.1601 & 0.1816 & -0.0164 & 0.0277 & -0.0255 & -0.3094 & 0.4081 & 0.227 & -0.0336 & 0.0405 \\
\hline$S T D V \_R O A$ & -0.1134 & -0.0342 & -0.1208 & 0.0519 & -0.1359 & -0.1731 & 0.1816 & 0.3371 & -0.0451 & 0.0793 \\
\hline$N \_A N A L Y S T$ & 0.0895 & 0.008 & 0.1205 & -0.1023 & 0.1555 & 0.0016 & 0.0117 & -0.2571 & 0.3793 & -0.3069 \\
\hline INST_HOLDING & 0.0223 & -0.0462 & 0.0934 & 0.1707 & 0.0376 & 0.1819 & -0.1686 & -0.0438 & 0.1104 & -0.2778 \\
\hline
\end{tabular}

\begin{tabular}{|c|c|c|c|c|c|c|c|c|}
\hline & $S I Z E$ & $M B$ & HORIZON & $C h \_E P S$ & $L O S S$ & $S T D V_{-} R O A$ & $N \_A N A L Y S T$ & $\begin{array}{c}I N S T \_H O \\
L D I N G\end{array}$ \\
\hline$S I Z E$ & 1 & & & & & & & \\
\hline$M B$ & 0.2455 & 1 & & & & & & \\
\hline HORIZON & 0.0003 & 0.0189 & 1 & & & & & \\
\hline
\end{tabular}




\begin{tabular}{lccccccc} 
Ch_EPS & 0.006 & -0.0101 & 0.0111 & 1 & & & \\
LOSS & -0.1666 & -0.1107 & -0.0009 & 0.0916 & 1 & & \\
STDV_ROA & -0.1575 & 0.1152 & -0.0024 & 0.0156 & 0.2559 & 1 & 1 \\
N_ANALYST & 0.483 & -0.0244 & -0.08 & -0.0442 & 0.0289 & -0.0294 & 0.1171 \\
INST_HOLDING & 0.2124 & 0.0126 & -0.0423 & 0.0171 & -0.0624 & -0.0922 & 1 \\
\hline
\end{tabular}


TABLE 2.3 (a)

Regression Analyses of Forecast Accuracy and Forecast Dispersion on Leverage

\begin{tabular}{|c|c|c|c|c|c|c|c|c|}
\hline & \multicolumn{4}{|c|}{ Dep Var: FORECAST ACCURACY } & \multicolumn{4}{|c|}{ Dep Var: FORECAST DISPERSION } \\
\hline VARIABLES & Coefficient & $t$-stat & Coefficients & $t$-stat & Coefficients & $t$-stat & Coefficients & $t$-stat \\
\hline$T L E V$ & $-1.131 * * *$ & -19.106 & & & $0.766 * * *$ & 16.577 & & \\
\hline FINLEV & & & $-1.046 * * *$ & -15.427 & & & $0.778 * * *$ & 14.479 \\
\hline OPLLEV & & & $-1.356 * * *$ & -13.967 & & & $0.725 * * *$ & 10.265 \\
\hline$S I Z E$ & $0.344 * * *$ & 34.491 & $0.348 * * *$ & 34.760 & $-0.131 * * *$ & -20.450 & $-0.130 * * *$ & -20.380 \\
\hline$M B$ & $0.037 * * *$ & 13.746 & $0.037 * * *$ & 13.603 & $-0.016^{* * *}$ & -9.778 & $-0.015^{* * *}$ & -9.545 \\
\hline HORIZON & $-0.047 * *$ & -2.030 & $-0.046^{* *}$ & -1.977 & $-0.027 *$ & -1.747 & $-0.027 *$ & -1.732 \\
\hline$C h \_E P S$ & -0.002 & -0.022 & 0.003 & 0.028 & $0.901 * * *$ & 6.243 & $0.901 * * *$ & 6.244 \\
\hline$L \overline{O S S}$ & $-0.839 * * *$ & -31.385 & $-0.843 * * *$ & -31.572 & $0.766 * * *$ & 26.575 & $0.768 * * *$ & 26.613 \\
\hline$S T D V \_R O A$ & $-0.878 * * *$ & -10.166 & $-0.879 * * *$ & -10.211 & $0.368 * * *$ & 4.849 & $0.365^{* * *}$ & 4.806 \\
\hline$N \_A N \bar{A} L Y S T$ & $-0.164 * * *$ & -7.511 & $-0.165 * * *$ & -7.556 & $0.112 * * *$ & 7.635 & $0.112 * * *$ & 7.616 \\
\hline Constant & $4.542 * * *$ & 21.341 & $4.541^{* * *}$ & 21.252 & $0.909 * * *$ & 8.177 & $0.912 * * *$ & 8.205 \\
\hline Year FE & Yes & & Yes & & Yes & & Yes & \\
\hline Industry FE & Yes & & Yes & & Yes & & Yes & \\
\hline Observations & 23,584 & & 23,584 & & 23,584 & & 23,584 & \\
\hline R-squared & 0.345 & & 0.346 & & 0.255 & & 0.254 & \\
\hline
\end{tabular}

Sample includes firm-year observations with at least three unique analyst forecasts from 1989 to 2017. The variable of interest, TLEV, FINLEV and OPLLEV are respectively defined as the ratio of total liability (LT) to total assets (AT), financial debt $(D L C+D L T T)$ to total assets $(A T)$, and operating liability leverage ( $L T-D L C-D L T T)$ to total assets $(A T)$. All variables in the table are defined in Appendix B. Industry dummies capture industry fixed effects with twodigit SIC code classifications. Standard errors are robust and clustered by firm (gvkey). Continuous variables are winsorized at top and bottom 1 percent. $* * *, * *$, and $*$ respectively denote significance at $1 \%, 5 \%$, and $10 \%$. 
TABLE 2.3 (b)

Regression Analyses of Firm-specific Information Environment on Leverage

\begin{tabular}{|c|c|c|c|c|c|c|c|c|c|c|c|c|}
\hline \multirow[b]{2}{*}{ VARIABLES } & \multicolumn{4}{|c|}{ Dep Var: IDVOL } & \multicolumn{4}{|c|}{ Dep Var: SYNCH } & \multicolumn{4}{|c|}{ Dep Var: PIN } \\
\hline & Coeff & $t$-stat & Coeff & $t$-stat & Coeff & $t$-stat & Coeff & $t$-stat & Coeff & $t$-stat & Coeff & $t$-stat \\
\hline$T L E V$ & $-0.005 * * *$ & -5.955 & & & -0.016 & -0.326 & & & 0.001 & 0.182 & & \\
\hline FINLEV & & & $-0.005 * * *$ & -5.288 & & & -0.017 & -0.313 & & & 0.003 & 0.721 \\
\hline OPLLEV & & & $-0.005 * * *$ & -3.985 & & & -0.011 & -0.140 & & & -0.004 & -0.772 \\
\hline$S I Z E$ & $-0.011 * * *$ & -61.156 & $-0.011 * * *$ & -60.582 & $0.216 * * *$ & 20.136 & $0.216 * * *$ & 19.937 & $-0.011 * * *$ & -16.163 & $-0.011 * * *$ & -15.670 \\
\hline$T R V O L$ & $0.008 * * *$ & 40.732 & $0.008 * * *$ & 40.583 & $0.093 * * *$ & 8.325 & $0.093 * * *$ & 8.319 & $-0.010^{* * *}$ & -11.943 & $-0.010 * * *$ & -11.934 \\
\hline N_ANALYST & $-0.001 * * *$ & -3.588 & $-0.001 * * *$ & -3.592 & $0.097 * * *$ & 5.187 & $0.097 * * *$ & 5.178 & $-0.003 * * *$ & -2.619 & $-0.003 * * *$ & -2.693 \\
\hline$S \bar{P} 500$ & $-0.005 * * *$ & -11.697 & $-0.005 * * *$ & -11.594 & 0.035 & 1.263 & 0.034 & 1.257 & $-0.008 * * *$ & -5.330 & $-0.007 * * *$ & -5.152 \\
\hline$S T D V \_R O A$ & $0.030 * * *$ & 23.035 & $0.030 * * *$ & 23.017 & -0.086 & -1.426 & -0.086 & -1.428 & $0.046^{* * *}$ & 4.052 & $0.046 * * *$ & 4.043 \\
\hline Constant & $-0.025 * * *$ & -7.811 & $-0.025 * * *$ & -7.747 & $-3.723 * * *$ & -12.234 & $-3.724 * * *$ & -12.232 & $0.428 * * *$ & 28.640 & $0.429 * * *$ & 28.253 \\
\hline Year FE & Yes & & Yes & & Yes & & Yes & & Yes & & Yes & \\
\hline Industry FE & Yes & & Yes & & Yes & & Yes & & Yes & & Yes & \\
\hline Observations & 21,437 & & 21,437 & & 21,502 & & 21,502 & & 6,461 & & 6,461 & \\
\hline R-squared & 0.674 & & 0.674 & & 0.408 & & 0.408 & & 0.550 & & 0.550 & \\
\hline
\end{tabular}

Sample includes firm-year observations with at least three unique analyst forecasts from 1989 to 2017 . The variable of interest, TLEV, FINLEV and OPLLEV are respectively defined as the ratio of total liability (LT) to total assets (AT), financial debt $(D L C+D L T T)$ to total assets $(A T)$, and operating liability leverage ( $L T-D L C-D L T T)$ to total assets $(A T)$. All variables in the table are defined in Appendix B. Industry dummies capture industry fixed effects with twodigit SIC code classifications. Standard errors are robust and clustered by firm ( $g v k e y)$. Continuous variables are winsorized at top and bottom 1 percent. $* * * * *$, and * respectively denote significance at $1 \%, 5 \%$, and $10 \%$. 
TABLE 2.4 (a)

Regression Analyses of Forecast Accuracy and Forecast Dispersion on Estimated and Contractual Operating Liability Leverage

\begin{tabular}{|c|c|c|c|c|c|c|c|c|}
\hline & \multicolumn{4}{|c|}{ Dep Var: FORECAST ACCURACY } & \multicolumn{4}{|c|}{ Dep Var: FORECAST DISPERSION } \\
\hline VARIABLES & Coefficient & $t$-stat & Coefficients & $t$-stat & Coefficients & t-stat & Coefficients & $t$-stat \\
\hline ESTOPL & $-1.328 * * *$ & -7.687 & $-1.386 * * *$ & -7.995 & $0.471 * * *$ & 3.800 & $0.520 * * *$ & 4.159 \\
\hline CONOPL & $-1.420 * * *$ & -12.937 & $-1.475 * * *$ & -13.426 & $0.825 * * *$ & 10.775 & $0.829 * * *$ & 10.707 \\
\hline SIZE & $0.349 * * *$ & 34.686 & $0.340 * * *$ & 32.291 & $-0.132 * * *$ & -20.573 & $-0.128 * * *$ & -19.137 \\
\hline FINLEV & $-1.043 * * *$ & -15.392 & $-1.030 * * *$ & -14.964 & $0.772 * * *$ & 14.406 & $0.737 * * *$ & 13.502 \\
\hline$M B$ & $0.037 * * *$ & 13.479 & $0.037 * * *$ & 13.443 & $-0.015 * * *$ & -9.247 & $-0.014 * * *$ & -8.982 \\
\hline HORIZON & $-0.046^{* *}$ & -1.976 & $-0.050 * *$ & -2.170 & $-0.027 *$ & -1.740 & -0.022 & -1.416 \\
\hline$C h \_E P S$ & 0.004 & 0.034 & -0.066 & -0.603 & $0.898 * * *$ & 6.220 & $0.831 * * *$ & 5.793 \\
\hline LOSS & $-0.845 * * *$ & -31.660 & $-0.853 * * *$ & -30.710 & $0.770 * * *$ & 26.650 & $0.741 * * *$ & 25.947 \\
\hline$S T D V \_R O A$ & $-0.880 * * *$ & -10.224 & $-0.761 * * *$ & -6.109 & $0.367 * * *$ & 4.828 & $0.502 * * *$ & 4.317 \\
\hline$N \_A N A L Y S T$ & $-0.164 * * *$ & -7.527 & $-0.143^{* * *} *$ & -6.547 & $0.111 * * *$ & 7.552 & $0.098 * * *$ & 6.661 \\
\hline TANNGIBILITY & & & $-0.204 * * *$ & -2.757 & & & $0.107 * *$ & 2.047 \\
\hline$B I G 4$ & & & $0.052 * *$ & 1.968 & & & -0.015 & -0.900 \\
\hline DIVPAYER & & & $0.053 * *$ & 2.049 & & & $-0.044 * * *$ & -2.648 \\
\hline$S T D \_C F O$ & & & $-0.286^{*}$ & -1.794 & & & -0.225 & -1.539 \\
\hline$A C \overline{C R} U A L S$ & & & $-0.296 * * *$ & -2.627 & & & -0.003 & -0.030 \\
\hline Constant & $4.547 * * *$ & 21.331 & $4.662 * * *$ & 20.683 & $0.904 * * *$ & 8.055 & $0.929 * * *$ & 7.098 \\
\hline Year FE & Yes & & Yes & & Yes & & Yes & \\
\hline Industry FE & Yes & & Yes & & Yes & & Yes & \\
\hline Observations & 23,584 & & 22,892 & & 23,584 & & 22,892 & \\
\hline R-squared & 0.346 & & 0.348 & & 0.255 & & 0.253 & \\
\hline
\end{tabular}

Sample includes firm-year observations with at least three unique analyst forecasts from 1989 to 2017. The variable of interest, ESTOPL, and CONOPL are respectively defined as the ratio of estimated operating liability leverage to total assets $(A T)$ and contractual operating liability leverage to total assets $(A T)$. All variables in the table are defined in Appendix B. Industry dummies capture industry fixed effects with two-digit SIC code classifications. Standard errors are robust and clustered by firm (gvkey). Continuous variables are winsorized at top and bottom 1 percent. ***, $* *$, and * respectively denote significance at $1 \%, 5 \%$, and $10 \%$. 
TABLE 2.4 (b)

Regression Analyses of Firm-specific Information Environment on Estimated and Contractual Operating Liability Leverage

\begin{tabular}{|c|c|c|c|c|c|c|c|c|c|c|c|c|}
\hline & \multicolumn{4}{|c|}{ Dep Var: IDVOL } & \multicolumn{4}{|c|}{ Dep Var: SYNCH } & \multicolumn{4}{|c|}{ Dep Var: PIN } \\
\hline VARIABLES & Coeff & $t$-stat & Coeff & $t$-stat & Coeff & $t$-stat & Coeff & $t$-stat & Coeff & $t$-stat & Coeff & $t$-stat \\
\hline ESTOPL & $-0.007 * * *$ & -2.797 & $-0.005 * *$ & -2.094 & 0.014 & 0.102 & 0.010 & 0.068 & -0.009 & -0.532 & -0.014 & -0.824 \\
\hline CONOPL & $-0.005 * * *$ & -3.435 & $-0.005 * * *$ & -3.314 & -0.016 & -0.181 & -0.025 & -0.271 & -0.004 & -0.805 & -0.004 & -0.778 \\
\hline SIZE & $-0.011 * * *$ & -60.580 & $-0.011 * * *$ & -59.677 & $0.216 * * *$ & 19.895 & $0.221 * * *$ & 20.211 & $-0.011 * * *$ & -15.658 & $-0.011 * * *$ & -15.464 \\
\hline FINLEV & $-0.005 * * *$ & -5.290 & $-0.004 * * *$ & -4.361 & -0.017 & -0.303 & -0.054 & -0.960 & 0.003 & 0.693 & 0.003 & 0.698 \\
\hline$T R V O L$ & $0.008 * * *$ & 40.590 & $0.008 * * *$ & 39.925 & $0.093 * * *$ & 8.322 & $0.086 * * *$ & 7.496 & $-0.010 * * *$ & -11.930 & $-0.010 * * *$ & -11.715 \\
\hline$N \_A N A L Y S T$ & $-0.001 * * *$ & -3.599 & $-0.001 * * *$ & -3.509 & $0.097 * * *$ & 5.182 & $0.090 * * *$ & 4.765 & $-0.003 * * *$ & -2.696 & $-0.003 * *$ & -2.414 \\
\hline SP500 & $-0.005 * * *$ & -11.614 & $-0.005 * * *$ & -10.967 & 0.034 & 1.256 & 0.014 & 0.495 & $-0.007 * * *$ & -5.146 & $-0.007 * * *$ & -5.111 \\
\hline$S T D V \_R O A$ & $0.030 * * *$ & 23.015 & $0.029 * * *$ & 20.472 & -0.086 & -1.434 & -0.077 & -1.164 & $0.046 * * *$ & 4.029 & $0.048 * * *$ & 3.809 \\
\hline HERFIN & & & $0.001^{*}$ & 1.912 & & & -0.021 & -0.667 & & & $0.007 * * *$ & 2.647 \\
\hline NFIRM & & & $0.007 * * *$ & 9.531 & & & $-0.233 * * *$ & -4.712 & & & $0.017 * * *$ & 3.135 \\
\hline ROACORR & & & $-0.001 * *$ & -2.120 & & & $0.165 * * *$ & 8.207 & & & 0.001 & 0.760 \\
\hline Constant & $-0.025 * * *$ & -7.761 & $-0.058 * * *$ & -9.383 & $-3.724 * * *$ & -12.241 & $-2.683 * * *$ & -5.738 & $0.429 * * *$ & 28.255 & $0.316 * * *$ & 8.516 \\
\hline Year FE & Yes & & Yes & & Yes & & Yes & & Yes & & Yes & \\
\hline Industry FE & Yes & & Yes & & Yes & & Yes & & Yes & & Yes & \\
\hline Observations & 21,437 & & 20,590 & & 21,502 & & 20,652 & & 6,461 & & 6,304 & \\
\hline R-squared & 0.674 & & 0.670 & & 0.408 & & 0.417 & & 0.550 & & 0.553 & \\
\hline
\end{tabular}

Sample includes firm-year observations with at least three unique analyst forecasts from 1989 to 2017. The variable of interest, ESTOPL, and CONOPL are respectively defined as the ratio of estimated operating liability leverage to total assets $(A T)$ and contractual operating liability leverage to total assets $(A T)$. All variables in the table are defined in Appendix B. Industry dummies capture industry fixed effects with two-digit SIC code classifications. Standard errors are robust and clustered by firm ( $g v k e y)$. Continuous variables are winsorized at top and bottom 1 percent. ***, $* *$, and $*$ respectively denote significance at $1 \%, 5 \%$, and $10 \%$. 
TABLE 2.5

Regression Analyses of Forecast Accuracy and Forecast Dispersion on Estimated and Contractual Operating Liability Leverage

Panel A: Rated vs. Unrated

\begin{tabular}{|c|c|c|c|c|c|c|c|c|}
\hline \multirow[b]{3}{*}{ VARIABLES } & \multicolumn{4}{|c|}{ Dep Var: FORECAST ACCURACY } & \multicolumn{4}{|c|}{ Dep Var: FORECAST DISPERSION } \\
\hline & \multicolumn{2}{|c|}{ Unrated } & \multicolumn{2}{|c|}{ Rated } & \multicolumn{2}{|c|}{ Unrated } & \multicolumn{2}{|c|}{ Rated } \\
\hline & Coefficient & $t$-stat & Coefficients & $t$-stat & Coefficients & $t$-stat & Coefficients & $t$-stat \\
\hline ESTOPL & $-1.411 * * *$ & -6.205 & $-1.122 * * *$ & -6.754 & $0.638 * * *$ & 3.847 & $0.415 * * *$ & 3.399 \\
\hline CONOPL & $-1.281 * * *$ & -10.822 & $-1.243 * * *$ & -11.655 & $0.828 * * *$ & 9.594 & $0.730 * * *$ & 9.295 \\
\hline SIZE & $0.340 * * *$ & 34.314 & $0.412 * * *$ & 40.438 & $-0.136 * * *$ & -18.832 & $-0.159 * * *$ & -21.264 \\
\hline FINLEV & $-0.969 * * *$ & -10.577 & $-0.795 * * *$ & -11.132 & $0.677 * * *$ & 10.145 & $0.621 * * *$ & 11.813 \\
\hline$M B$ & $0.035 * * *$ & 9.753 & $0.031 * * *$ & 10.510 & $-0.014 * * *$ & -5.329 & $-0.012 * * *$ & -5.613 \\
\hline HORIZON & 0.003 & 0.099 & $-0.093 * * *$ & -3.300 & -0.036 & -1.531 & -0.024 & -1.147 \\
\hline$C h \_E P S$ & -0.132 & -0.949 & 0.097 & 0.656 & $0.766 * * *$ & 7.534 & $1.043 * * *$ & 9.587 \\
\hline$L \overline{O S S}$ & $-0.777 * * *$ & -22.868 & $-0.837 * * *$ & -28.352 & $0.849 * * *$ & 34.280 & $0.685 * * *$ & 31.562 \\
\hline$S T D V \_R O A$ & $-1.287 * * *$ & -7.557 & $-0.697 * * *$ & -8.967 & $0.720 * * *$ & 5.796 & $0.326 * * *$ & 5.697 \\
\hline N_ANALYST & $-0.190 * * *$ & -7.765 & $-0.096 * * *$ & -3.684 & $0.090 * * *$ & 5.057 & $0.130 * * *$ & 6.721 \\
\hline Constant & $4.753 * * *$ & 17.083 & $3.886 * * *$ & 15.800 & $1.165^{* * *}$ & 5.745 & $0.939 * * *$ & 5.188 \\
\hline $\begin{array}{l}\text { Year FE } \\
\text { Industry FE } \\
\text { Observations } \\
\text { R-squared }\end{array}$ & $\begin{array}{c}\text { Yes } \\
\text { Yes } \\
11,481 \\
0.356\end{array}$ & & $\begin{array}{c}\text { Yes } \\
\text { Yes } \\
12,103 \\
0.353 \\
\end{array}$ & & $\begin{array}{c}\text { Yes } \\
\text { Yes } \\
11,481 \\
0.286\end{array}$ & & $\begin{array}{c}\text { Yes } \\
\text { Yes } \\
12,103 \\
0.239 \\
\end{array}$ & \\
\hline
\end{tabular}


Panel B: High vs. Low Analysts Following

\begin{tabular}{|c|c|c|c|c|c|c|c|c|}
\hline \multirow[b]{3}{*}{ VARIABLES } & \multicolumn{4}{|c|}{ Dep Var: FORECAST ACCURACY } & \multicolumn{4}{|c|}{ Dep Var: FORECAST DISPERSION } \\
\hline & \multicolumn{2}{|c|}{ Low Following } & \multicolumn{2}{|c|}{ High Following } & \multicolumn{2}{|c|}{ Low Following } & \multicolumn{2}{|c|}{ High Following } \\
\hline & Coefficient & $t$-stat & Coefficients & $t$-stat & Coefficients & $t$-stat & Coefficients & $t$-stat \\
\hline ESTOPL & $-1.211 * * *$ & -7.111 & $-1.377 * * *$ & -6.436 & $0.532 * * *$ & 4.032 & $0.348 * *$ & 2.426 \\
\hline CONOPL & $-1.371 * * *$ & -13.554 & $-1.436 * * *$ & -12.081 & $0.956 * * *$ & 12.194 & $0.685 * * *$ & 8.603 \\
\hline SIZE & $0.403 * * *$ & 47.605 & $0.293 * * *$ & 30.301 & $-0.147 * * *$ & -22.465 & $-0.114 * * *$ & -17.583 \\
\hline FINLEV & $-0.978 * * *$ & -15.568 & $-1.183 * * *$ & -14.475 & $0.785 * * *$ & 16.126 & $0.737 * * *$ & 13.460 \\
\hline$M B$ & $0.030 * * *$ & 10.156 & $0.042 * * *$ & 12.485 & $-0.012 * * *$ & -5.365 & $-0.018 * * *$ & -7.880 \\
\hline HORIZON & -0.031 & -1.115 & $-0.065^{*}$ & -1.936 & -0.028 & -1.328 & $-0.046 * *$ & -2.051 \\
\hline$C h \_E P S$ & 0.050 & 0.381 & -0.113 & -0.709 & $0.715 * * *$ & 7.004 & $1.184 * * *$ & 11.126 \\
\hline LOSS & $-0.867 * * *$ & -30.125 & $-0.776 * * *$ & -22.209 & $0.783 * * *$ & 35.136 & $0.749 * * *$ & 32.013 \\
\hline$S T D V \_R O A$ & $-0.753 * * *$ & -8.971 & $-0.995 * * *$ & -7.661 & $0.341 * * *$ & 5.253 & $0.424 * * *$ & 4.874 \\
\hline$N \_A N A L Y S T$ & 0.038 & 0.754 & $-0.270 * * *$ & -7.467 & $0.079 * *$ & 2.028 & $0.132 * * *$ & 5.427 \\
\hline Constant & $3.832 * * *$ & 15.950 & $5.445^{* * *}$ & 17.770 & $0.971 * * *$ & 5.217 & $0.867 * * *$ & 4.226 \\
\hline Year FE & Yes & & Yes & & Yes & & Yes & \\
\hline Industry FE & Yes & & Yes & & Yes & & Yes & \\
\hline Observations & 13,428 & & 10,156 & & 13,428 & & 10,156 & \\
\hline R-squared & 0.365 & & 0.326 & & 0.250 & & 0.279 & \\
\hline
\end{tabular}


Panel C: High vs. Low Institutional Shareholdings

\begin{tabular}{|c|c|c|c|c|c|c|c|c|}
\hline & \multicolumn{4}{|c|}{ Dep Var: FORECAST ACCURACY } & \multicolumn{4}{|c|}{ Dep Var: FORECAST DISPERSION } \\
\hline & \multicolumn{2}{|c|}{ Low Inst. } & \multicolumn{2}{|c|}{ High Inst. } & \multicolumn{2}{|c|}{ Low Inst. } & \multicolumn{2}{|c|}{ High Inst. } \\
\hline VARIABLES & Coefficient & $t$-stat & Coefficients & $t$-stat & Coefficients & $t$-stat & Coefficients & $t$-stat \\
\hline ESTOPL & $-1.244 * * *$ & -5.404 & $-1.383 * * *$ & -8.437 & $0.583 * * *$ & 3.288 & $0.376^{* * *}$ & 3.359 \\
\hline CONOPL & $-1.458 * * *$ & -13.322 & $-1.404 * * *$ & -12.901 & $0.957 * * *$ & 11.359 & $0.684 * * *$ & 9.206 \\
\hline SIZE & $0.358 * * *$ & 40.223 & $0.337 * * *$ & 35.614 & $-0.147 * * *$ & -21.421 & $-0.107 * * *$ & -16.652 \\
\hline FINLEV & $-1.167 * * *$ & -16.274 & $-0.929 * * *$ & -13.405 & $0.926 * * *$ & 16.770 & $0.591 * * *$ & 12.509 \\
\hline$M B$ & $0.034 * * *$ & 10.134 & $0.041 * * *$ & 13.534 & $-0.013 * * *$ & -5.127 & $-0.016^{* * * *}$ & -7.637 \\
\hline HORIZON & $-0.095 * * *$ & -2.884 & -0.012 & -0.440 & -0.022 & -0.874 & $-0.038 * *$ & -2.008 \\
\hline$C h \_E P S$ & -0.010 & -0.072 & -0.026 & -0.181 & $0.998 * * *$ & 9.030 & $0.783 * * *$ & 8.008 \\
\hline LOSS & $-0.916 * * *$ & -28.979 & $-0.714 * * *$ & -22.913 & $0.834 * * *$ & 34.256 & $0.674 * * *$ & 31.695 \\
\hline$S T D V \_R O A$ & $-0.607 * * *$ & -6.714 & $-1.076 * * *$ & -9.215 & $0.191 * * *$ & 2.752 & $0.550 * * *$ & 6.898 \\
\hline N_ANALYST & $-0.260 * * *$ & -9.419 & $-0.108 * * *$ & -4.698 & $0.139 * * *$ & 6.535 & $0.099 * * *$ & 6.344 \\
\hline Constant & $4.906^{* * *}$ & 19.829 & $4.486^{* * *}$ & 13.922 & $0.731 * * *$ & 3.839 & $1.130 * * *$ & 5.142 \\
\hline Year FE & Yes & & Yes & & Yes & & Yes & \\
\hline Industry FE & Yes & & Yes & & Yes & & Yes & \\
\hline Observations & 11,792 & & 11,792 & & 11,792 & & 11,792 & \\
\hline R-squared & 0.361 & & 0.314 & & 0.271 & & 0.242 & \\
\hline
\end{tabular}


Panel D: Small vs. Big Firm

\begin{tabular}{|c|c|c|c|c|c|c|c|c|}
\hline & \multicolumn{4}{|c|}{ Dep Var: FORECAST ACCURACY } & \multicolumn{4}{|c|}{ Dep Var: FORECAST DISPERSION } \\
\hline & \multicolumn{2}{|c|}{ Small } & \multicolumn{2}{|c|}{ Big } & \multicolumn{2}{|c|}{ Small } & \multicolumn{2}{|c|}{ Big } \\
\hline VARIABLES & Coefficient & $t$-stat & Coefficients & $t$-stat & Coefficients & $t$-stat & Coefficients & $t$-stat \\
\hline ESTOPL & $-1.083 * * *$ & -5.763 & $-1.295 * * *$ & -6.790 & $0.505 * * *$ & 3.439 & $0.343 * * *$ & 2.637 \\
\hline CONOPL & $-1.154 * * *$ & -10.234 & $-1.212 * * *$ & -10.955 & $0.753 * * *$ & 8.548 & $0.608 * * *$ & 8.055 \\
\hline SIZE & $0.511 * * *$ & 41.805 & $0.323 * * *$ & 31.687 & $-0.232 * * *$ & -24.350 & $-0.127 * * *$ & -18.224 \\
\hline FINLEV & $-0.886^{* * *}$ & -12.907 & $-0.943 * * *$ & -11.608 & $0.674 * * *$ & 12.573 & $0.599 * * *$ & 10.811 \\
\hline$M B$ & $0.022 * * *$ & 6.912 & $0.039 * * *$ & 11.724 & $-0.010 * * *$ & -3.971 & $-0.011 * * *$ & -4.697 \\
\hline HORIZON & $-0.114 * * *$ & -3.842 & 0.006 & 0.208 & -0.013 & -0.570 & $-0.035^{*}$ & -1.699 \\
\hline$C h \_E P S$ & 0.011 & 0.078 & -0.113 & -0.788 & $1.077 * * *$ & 9.724 & $0.741 * * *$ & 7.566 \\
\hline LOSS & $-0.837 * * *$ & -28.544 & $-0.729 * * *$ & -21.228 & $0.693 * * *$ & 30.245 & $0.832 * * *$ & 35.530 \\
\hline$S T D V \_R O A$ & $-0.640 * * *$ & -8.128 & $-1.513 * * *$ & -9.168 & $0.347 * * *$ & 5.631 & $0.668 * * *$ & 5.933 \\
\hline$N \_A N A L Y S T$ & $-0.067 * *$ & -2.285 & $-0.161 * * *$ & -7.151 & $0.142 * * *$ & 6.210 & $0.073 * * *$ & 4.730 \\
\hline Constant & $3.447 * * *$ & 13.657 & $4.586 * * *$ & 16.501 & $1.324 * * *$ & 6.713 & $1.205 * * *$ & 6.354 \\
\hline Year FE & Yes & & Yes & & Yes & & Yes & \\
\hline Industry FE & Yes & & Yes & & Yes & & Yes & \\
\hline Observations & 11,792 & & 11,792 & & 11,792 & & 11,792 & \\
\hline R-squared & 0.353 & & 0.327 & & 0.254 & & 0.277 & \\
\hline
\end{tabular}




\section{APPENDIX B}

Variable Definition:

TLEV: ratio of total liabilities (LT) to total assets (AT).

FINLEV: ratio of financial leverage (DLTT+DLC) to total assets.

OPLEV: ratio of operating liability leverage (LT-DLTT-DLC) to total assets.

ESTOPL: ratio of estimated operating liability leverage (DRC+DRLT+XACC) to total assets.

CONOPL: ratio of contractual operating liability leverage to total assets.

ACCURACY: negative one times log of absolute value difference between actual EPS and median forecasted EPS, scaled by fiscal year end stock price.

DISPERSION: standard deviation of analyst forecast scaled by the year end stock price (times 100).

IDVOL: probability of informed trading collected from Soren Hvidkjaer's website.

SYNCH: standard deviation of excess return calculated from weekly return regression.

$P I N$ : log-transformed $\mathrm{r}$-square of weekly return regressions.

SIZE: $\log$ of market value of equity of firm $i$ in year $t$.

$M B$ : ratio market value of equity to book value of equity in year $t$.

HORIZON: log of number of days between the forecast date and the earnings announcement date.

Ch_EPS: $\quad$ change in earnings-per-share, scaled by share price at the end of year $t-1$.

LOSS: indicator variable 1 , if negative earnings is reported in year $t, 0$ otherwise.

STDV_ROA: standard deviation of ROA (IB/AT) over last 5 years (with min 3 years of data).

$N \_A N A L Y S T: \quad \log$ of number of analysts following firm $i$ in year $t$.

INST_HOLDING: average percentage of shares owned by institutional investors throughout the year $t$.

OPLLEV: operating liability leverage, scaled by total assets, both at the end of year $t$.

TANGIBILITY: net property plant and equipment, scaled by total assets, both at the end of year $t-1$.

BIG4: indicator variable 1 , if firm $i$ is audited by a Big4 auditor in year $t$.

DIVPAYER: indicator variable 1 , if common stock dividend is paid in year $\mathrm{t}, 0$ otherwise.

STD_CFO: standard deviation of operating cash flows over last 5 years (min 3 years of data).

ACCRUALS: net income minus cash flow from operations in year $t$ scaled by total assets in $t-1$.

DEPREICATION: depreciation scaled by total assets in $t-1$.

$R N D$ : research and development expenses scaled by total assets in $t-1$.

$R N D \_D U M: \quad$ indicator variable 1 , if research and development expense is missing.

$M E D \_D E B T:$ industry media debt ratio for year $t-1$.

TRVOL: average daily stock turnover of firm $i$ in year $t$.

SP500: indicator variable 1 for S\&P 500 firms.

HERFIN: Herfindahl index for industry concentration based on sales. 
NFIRM: number of firms in each Fama-French 48 industry.

ROACORR: correlation between industry-average ROA and firm's ROA over past 12 quarters. 
ESSAY 3: DEBT COVENANT STRICTNESS AND FINANCIAL REPORTING

QUALITY

\section{INTRODUCTION}

A firm is viewed as a "nexus of contracts" among various contracting agents (Jensen and Meckling 1976). Prior studies have extensively evaluated the way a firm interacts with, and subsequently responds to, its contracting agents under various conditions. Specifically, studies have explored the how accounting information mitigates agency conflicts in designing efficient contracts (Armstrong, Guay and Weber 2010). In this study, I extend prior understanding of contracting relationships by documenting financial reporting outcomes of those relationships. In particular, I investigate how contracting relationship with a lender impacts its borrower's financial reporting quality.

Studies take contrasting perspectives as to how debt contracts affect financial reporting quality of a firm. In the one hand, debt contracts impose non-trivial monitoring costs on firms and improve financial reporting quality by limiting managerial discretion over financial reporting ${ }^{10}$ (Monitoring Hypothesis). On the other hand, to avoid costly covenant violations, managers exhibit myopic behavior by managing earnings and thus exacerbate financial reporting quality (Debt Covenant Hypothesis). Empirical evidence on whether covenants concerns motivates a manager's financial reporting decisions is also inconclusive. Studies argue that with greater monitoring and intervention in place, firm performance improves and profitability increases (Spyridopoulos 2015; Nini, Smith and

\footnotetext{
${ }^{10}$ One way of limiting managerial discretion is by restricting managers from switching accounting policies during the term of the contract. Another way of discipling managers is through increasing the frequency and intensity of bank inspections. Increased demand for timely loss recognition also complements lenders monitoring.
} 
Sufi 2012; Green 2018), and the likelihood of adopting conservative accounting increases (Khan and Lo 2017; Tan 2013; Nikolaev 2010). In contrast, studies report that firms are eager to avoid covenant violation and, as covenant threshold approaches, firms make accounting changes, such as income-increase accounting policies, higher total and working capital accruals, and increased accruals and real activities earnings management (Sweeney 1994; DeFond and Jiambalvo 1994; Franz, HassabElnaby, and Lobo 2014). Few studies, however, find little or no evidence of earnings management as means of satisfying covenant thresholds (Healy and Palepu 1990; DeAngelo et al. 1994; Benish 1999; Frankel and Litov 2007). Furthermore, in their survey, Graham et al. (2005) also find no support for the bond covenant hypothesis. Nonetheless, divergence in empirical findings in prior literature can be attributed to several measurement and design choices. To proxy for covenant restrictiveness, earlier works on debt covenant hypothesis use leverage ratio, while later studies chose a subset of covenants, e.g. current ratio and net worth ratio, and ignore others, which in turn under- or overreported covenants restrictiveness.

Similar to Wang (2017), I argue that among other features of a debt contract, covenant strictness has the most significant effect on a firm's operating and reporting decision. Tightly held covenants either contain shareholders' ex post rent seeking behavior or reflect true economic state of a borrower, for which future creditor intervention reduces (Demiroglu and James, 2010). Strictly designed covenant can also signal distress timely or prompt covenant violations and incentivize contract renegotiations (Wang, 2017; Prilmeier, 2017). As strict covenants set substantially stringent requirement on borrowers, lenders monitoring, or managerial opportunism should be related the restrictive level and type of debt covenants. Thus, I contend that restrictiveness of debt covenant offers the 
unique setting to study the link between covenant design and financial reporting quality ${ }^{11}$. To capture debt covenant strictness, I use a comprehensive measure of covenant strictness that uses full set of covenants used in debt contracts and mitigates measurement issues by incorporating various aspects of covenants, such as covenant intensity, slack, volatility and correlation.

Next, I argue that neither all debt covenants induce earnings management nor establish better monitoring. Christensen and Nikolaev (2012) identify covenants as capital covenants (balance sheet based) or performance covenants (income statement based) and hypothesize that capital covenants put restriction on borrower's total debt and thus align interest ex ante, while performance covenants allow lenders to intervene borrower's decisions and thus transfer control rights ex post. Demerjian (2011) argue that due to declining benefit of balance sheet to the debt contracting parties, the use of capital covenants has decreased over time, while inclusion of income statement covenants has remained constant. Christensen et al (2018) report that, as performance covenants facility greater creditor influence, lenders are more likely to choose performance covenants than capital covenants.

To empirically examine the association between covenant strictness and its impact on firm reporting quality, I conduct two sets of analyses. First, I use a fixed-effect based regression model to estimate the association between covenant strictness and borrower reporting quality. Next, I use separate measures of strictness for performance covenants

\footnotetext{
${ }^{11}$ The survey evidence reported in Graham, Harvey and Rajgopal (2005) suggests that debt covenants, in general, do not encourage earnings management. However, firms with binding constraints (strict covenants) consider covenants more important and hence are more likely to manage earnings.
} 
and capital covenants and repeat the analyses. I primarily use two proxies to capture a firm's financial reporting quality: absolute value of abnormal accruals estimated from the Modified Jones (1991) model, and absolute value of performance-matched abnormal accruals from by Kothari, Leone and Wasley (2005). Primary empirical findings indicate that debt covenant strictness in general does not affect financial reporting quality of a firm. However, when separate measures of strictness for performance covenant and capital covenants are used in the same regression, findings indicate that the strictness in capital covenants is positively associated with the absolute value of abnormal accruals suggesting poor financial reporting quality. However, a caveat of testing the effect of debt covenants on financial reporting quality is that the design of debt covenants and financial reporting quality are endogenously determined. Financial reporting quality can affect, and subsequently be affected by, debt contracts. I address this concern by using an instrumental variable (IV) based on the findings of Murfin (2012), who identifies lender-specific financial shock as a supply side determinant of debt covenants ${ }^{12}$. The underlying assumption of using lender-specific shock as an IV is that the change in loan contract strictness is driven by shocks that affect only the lead arrangers and is plausibly exogenous to borrower characteristics. The instrumental variable approach substantiates above findings.

\footnotetext{
${ }^{12}$ Due to endogenous nature of relationships among governance mechanisms, features of debt contracts and attributes of firms' accounting systems, Armstrong et al (2010) call for more research in identifying and using exogenous shocks in that establish causality in these relationships.
} 
The paper develops as follows. Section II reflects of related literature and hypotheses. Section III explains the empirical framework, regression variables and data. Section IV presents regression results, and section V concludes the paper.

\section{RELATED LITERATURE AND HYPOTHESIS DEVELOPMENT}

As a key financier, debtholders seek to ensure timely repayment of the principal and interest by closely monitoring and consistently evaluating borrower's economic state. Unlike equity holders, debtholders suffer from borrowers' economic losses but do not enjoy from economic gains. Thus, debtholders define financial tripwires, known as debt covenants, that limit managerial ability to contain shareholders' rent seeking behavior and that shift control rights to the lender during bad economic state of the firm (Jensen and Meckling, 1976). Prior studies document the role of debt covenants as a device to mitigate lender-shareholder conflict by limiting moral hazard pursuits such as risk shifting, underinvestment, and asset substitution (Jensen and Meckling 1976; Myers 1977). However, contracting relationship between lender and borrower is inherently incomplete partly due to the inability of participants to define all future circumstances that generates the potential for post-contractual opportunism (Christensen, Nikolaev and Wittenberg-Moerman 2016). As such, lenders impose stricter debt covenants that increase the probability of violation and strengthen contracting efficiency by transferring control rights quickly and correctly between borrowers and lenders.

With stricter covenants in place, borrowers are eager to avoid covenant violations (Graham, Harvey and Rajgopal 2005), and lenders are keen to curb borrowers' use of inappropriate means to achieve the avoidance. For borrowers, covenant violations are 
costly with direct consequences of renegotiation costs and default costs. Indirect consequences of covenant violation include changes in firms' investment and financing strategies, corporate restructuring and executive turnovers (Chava and Roberts 2008; Bhaskar, Krishnan and Yu 2017; Gao, Khan and Tan 2017; Jiang and Zhou 2017). Covenant violations also lead to large negative stock price reactions ${ }^{13}$, increases in interest rates, and difficulty in securing further financing (Beneish and Press, 1993, 1995; Roberts and Sufi, 2009). To avoid negative consequences of covenant violation, borrowers are expected to behave in a way that affirms meeting covenant thresholds. However, borrowers' ability to opportunistically meet covenants are limited by lenders monitoring. Stricter debt convents thus induce two offsetting effects on financial reporting quality: on the one hand tight convents limit managerial discretion by extended monitoring and on the other hand tight convents encourage managerial manipulation to avoid covenant violation.

\section{Monitoring Hypothesis:}

Stricter debt covenants can improve financial reporting quality through increased demand for accounting conservatism (Watts and Zimmerman 1986; Ball, Robin and Sadka 2008), and intent monitoring. It is widely discussed in the literature that debt works as a governance mechanism in the firm (Harris and Raviv 1991) and debt covenants play a key role in creditors' monitoring activities. Stricter debt covenants allocate contingent control rights quickly to the creditor (Jensen and Meckling 1976) and hence establish intense monitoring over borrower's performance. Due to greater monitoring and actions imposed

\footnotetext{
${ }^{13}$ Ertan and Karolyi (2014) estimate that equity holders' ex ante expected cost from covenant violation is $8.40 \%$ of equity value, while Beneish and Press (1993) report that ex post cost of technical default ranges between $1.2 \%$ and $2.0 \%$ of the firm's market value of equity.
} 
by creditors, firm performance improves with stricter covenants (Spyridopoulos 2015), and even after covenant violations (Nini et al. 2012). Using a dynamic model, Green (2018) finds that restrictive covenants are essential for high leverage firms to generate value.

With increased monitoring and intervention, managerial discretion over financial reporting gets curtailed and thus financial reporting outcomes are also expected to be improved. Using a natural experiment, Fang et al. (2015) show that external monitoring by short sellers reduce accruals-based earnings management. Gul and Goodwin (2010) argue that short-term debt lowers audit fees as it establishes more monitoring and better governance, decreasing auditors' concerns about a borrower's misreporting risk. Crosscountry studies complement these findings and document that stronger investor rights are associated with lower levels of earnings management (Leuz et al. 2003; Burgstahler et al. 2006). Taking a sample of debt covenant violations, Tan (2013) finds that firms adopt conservative reporting immediately after covenant violations and the effect lasts over at least eight quarters. Using a sample of public debt issues, Nikolaev (2010) report a positive association between covenants in public debt contracts and timely loss recognition. Khan and Lo (2017) find that borrowers' asymmetric recognition of economic losses increases as lending standards tightens. Using an Indian setting, Bhambwani (2017) similarly report that intensely monitored firms reduce their management of accruals as well as real activities. Higher financial reporting quality in the presence of increased monitoring facilitates better re-negotiations and favorable contractual terms and firms are thus encouraged to improve their reporting quality. 
However, increased monitoring does necessarily lead to better quality financial reporting. Studies argue that enhanced monitoring reduces information asymmetry and thus decreases lenders' demand for higher quality financial reporting. Vashishtha (2014) shows that, in the presence of increased bank monitoring, firms tend to reduce management disclosures following covenant violations. Erkens, Subramanyam, and Zhang (2014) investigate lender representation on a borrower's board as an alternate monitoring mechanism and show that such monitoring leads to a reduction in the borrower's conservatism. Also, better quality financial reporting is not costless to borrowers. For instance, Zhang (2008) finds that firms that the probability of violating covenant increases when firms adopt conservative accounting. Li (2009) and Gigler et al. (2009) suggest that costs associated with violation covenants for adopting conservative accounting (false positives) are greater than the benefits of timely loss recognition, and thus, conservative reporting is suboptimal for the borrower. Given the costs and consequences of covenant violation accelerated through conservative accounting, borrowers may reduce its reporting quality, which is consistent with the debt covenant hypothesis.

\section{Debt Covenant Hypothesis:}

The debt covenant hypothesis suggests that managers adopt financial reporting decisions that help them avoid violation of debt covenants (Watts and Zimmerman 1986, 1990). Empirical studies also support debt covenant hypothesis by documenting that managers are more likely to avoid covenant violation by engaging in various forms of earnings management. For instance, Sweeney (1994) reports that firms adopt incomeincreasing accounting choices when they approach a debt covenant violation. Using a 
sample of firms that violated debt covenants, DeFond and Jiambalvo (1994) report that total accruals and working capital accruals are significantly positive in the year before and in the year of the covenant violation. Dichev and Skinner (2002) show a significantly large number of firms reporting financial performance that just meet or beat covenant threshold. Beatty and Weber (2003) similarly report that firms adopt income-increasing accounting choices when there are binding debt covenants. A recent study by Franz, HassabElnaby and Lobo (2014) complement previous findings by establishing a positive association between proximity of covenant violations and both accruals and real activities management.

Few studies however find little or no support for debt covenant hypothesis. Healy and Palepu (1990) find no evidence that firms make accounting changes when creditor restriction is in place. DeAngelo et al. (1994) also report little difference in accruals in firms with and without restrictive covenants. Beneish (1999) does not find evidence of earnings overstatement in firms facing SEC enforcement actions motivated by concerns of debt covenant violations. Similarly, Frankel and Litov (2007) report that borrower conservatism is not associated with strictness of debt covenants. In addition, survey evidence reported in Graham, Harvey and Rajgopal (2005) suggests that debt covenants, in general, do not encourage earnings management. However, they report that firms with binding constraints (strict covenants) consider covenants more important and hence are more likely to manage earnings. 


\section{Hypothesis:}

In the presence of strict debt covenants, lenders and borrowers, being on the two sides of debt contracts, generate two contrasting effects on borrower's financial reporting quality. Lenders contribute to the improvement of borrowers financial reporting quality by (i) demanding timelier disclosure and recognition of economic losses and (ii) narrowly monitoring and exerting influence over borrowers' operation. Borrowers, however, have incentives to manipulate financial statements to avoid covenant breaching. In aggregate, it is not clear which of these two contrasting effects are likely to dominate, which leads to our first hypothesis (in alternative form).

\section{H1: Debt contracts with strict covenants increase (decrease) financial reporting} quality.

If financial reporting quality varies with the covenant tightness, then I also expect the findings to be further affected by the type of covenants. Christensen and Nikolaev (2012) identify debt covenants as performance covenants (income statement based) and capital covenants (balance sheet based) and contend that capital covenants put restriction on borrower's debt and thus align the interest among contracting parties, whereas performance allow creditors to proactively interfere with the borrower's decisions and thus serve as tripwires. Studies also argue that contracts embedded with the performance covenants are more likely to trigger renegotiations (Christensen and Nikolaev 2012, Wang 2017), which in turn provokes greater incentives among borrowers to avoid covenant breaching. Demerjian (2011) argue that balance sheet has become less useful for contracting and hence the use of balance sheet covenants has decreased over time, while 
inclusion of income statement covenants has remained constant. Christensen et al (2018) similarly report that in response to adverse financial shocks, lenders shift towards performance-based covenants as a means of gaining more influence over borrowers' operation. Therefore, I hypothesize that the effects of strictness of performance covenants and that of capital covenants would be different on financial reporting outcome of a firm.

H2: The effect of covenant strictness on financial reporting quality is different for performance-based covenants than for capital-based covenants.

\section{METHOD}

\section{Measure of Covenant Strictness}

To estimate the effect of covenant strictness on firm financial reporting quality, I use a comprehensive measure of covenant strictness from Demerian and Owen (2016), probability of covenant violation, which capture the probability of violating at least one covenant over the next quarter ${ }^{14}$. This measure has several advantages compared with alternative proxies of covenant tightness. First, instead of focusing on one or two financial covenants, probability of covenant violation uses the full set of covenants available in Dealscan. Second, it develops standard covenant definitions and reduces measurement error as acknowledged by Murfin (2012). Third, this measure combines various features of covenants that potentially determine tightness, including the total number of covenants (intensity), the distance between actual value and covenant threshold (slack), and the variance-covariance of underlying financial ratios. Demerjian and Owens (2016) posit that

\footnotetext{
14 Demerjian and Owen (2016) closely follow the approach suggested by Murfin (2012), but offer substantial improvements in mitigating measurement error as acknowledged by Murfin (2012).
} 
their measure has predictive power of covenant violation than alternative measures ${ }^{15}$. To test my second hypothesis, I group covenants into performance and capital covenants and calculate probability for defaults for two separate groups.

\section{Measures of Financial Reporting Quality (FRQ):}

In absence of a universally accepted measure $F R Q$, studies have identified different attributes that are associated with or reflective of reporting quality. Following prior studies, I construct measures that capture multiple dimensions of reporting quality and that generalize my findings. The use of alternative proxies also mitigates idiosyncratic measurement error. My first set of proxies captures accruals quality, calculated from a modified Jones (1991) model, and performance matched discretionary accruals model as suggested by Kothari et al. (2005).

The first accrual quality measure is based on Jones (1991). Following Dechow, Sloan, and Sweeney (1995), I use the modified version of Jones (1991) and estimate the following cross-sectional regression for each year and industry (two-digit SIC).

$$
\frac{T A_{i, t}}{\operatorname{Asset}_{i, t-1}}=\gamma_{0}+\gamma_{1} \frac{1}{\operatorname{Assets}_{i, t-1}}+\gamma_{2} \frac{\Delta \operatorname{Rev}_{i, t}}{\operatorname{Assets}_{i, t-1}}+\gamma_{3} \frac{P P E}{\operatorname{Asset}_{i, t-1}}+\varepsilon_{i, t}
$$

where $T A_{i, t}$ is total accruals (income from continuing operations $(I B)$ less operating cash flow $(O A N C F), \triangle R e v_{i, t}$ is change in revenue $(S A L E)$ from prior year, and $P P E_{i, t}$ is the property, plant, and equipment (PPEGT) for firm $i$ in year $t$. Next, I use coefficient estimates from the above equation to calculate expected accruals.

\footnotetext{
15 To provide evidence of usefulness of their measure, Demerjian and Owens (2016) revisited Frankel and Litov (2007). Replacing Frankel and Litov's (2007) measure with probability of violation, Demerjian and Owens (2016) document significant results in the predicted direction.
} 


$$
E A_{i, t}=\hat{\gamma}_{0}+\hat{\gamma}_{1} \frac{1}{\operatorname{Assets}_{i, t-1}}+\hat{\gamma}_{2} \frac{\left(\Delta \operatorname{Rev}_{i, t}-\Delta A R_{i, t}\right)}{\operatorname{Assets}_{i, t-1}}+\hat{\gamma}_{3} \frac{P P E}{\operatorname{Assets}_{i, t-1}}
$$

where $E A_{i, t}$ is expected accruals, and $\triangle A R_{i, t}$ is change in accounts receivables (RECT) from prior year for firm $i$ in year $t$. Discretionary accruals is the absolute value difference between total accruals and estimated accruals, $A b s D A C C_{i, t}=\operatorname{abs}\left(\frac{T A_{i, t}}{\operatorname{Assets}_{i, t-1}}-E A_{i, t}\right)$, which reflects the amount of earnings exposed to managerial discretion for earnings management.

The second proxy for accrual quality is discretionary accruals adjusted for firm performance, $P M D A C C_{i, t}$, calculated from an industry-specific regression model (Kothari et al. 2005). Following the approach used in Francis, LaFond, Olsson, and Schipper (2005), I first estimate expected accruals within two-digit industry by year, and then calculate performance-adjusted abnormal accruals as the difference between firm $i$ 's abnormal accrual and the median abnormal accrual of firm $i$ 's industry-ROA decile, where the median industry-ROA decile is calculated excluding firm $i$.

\section{Baseline Regression Model}

To examine the relation between debt covenant strictness and financial reporting quality, I estimate the following model:

$$
\begin{aligned}
& \text { AbsDACC }_{i, t} \text { or PMDACC } i, t=\beta_{0}+\beta_{1} \text { CovStrictness }_{i, t}+ \\
& \sum_{k} \beta_{k} \text { FirmControls }+\sum_{l} \beta_{l} \text { LoanControls }+ \text { IndustryFE }+ \text { YearFE }+\varepsilon
\end{aligned}
$$


Independent variable of interest is covenant strictness (PVIOL), measured as probability of covenant violation. I also replace $P V I O L$ with $P V I O L_{-} P$ and $P V I O L_{-} C$, respectively indicating probability of violating performance covenant and capital covenant. I include two sets of control variables that are associated with covenant strictness and firm financial reporting quality. Specifically, loan-level controls include natural logarithm of dollar amount of loan (LOANAMT), natural logarithm of maturity of loan in months (LOANMAT), all-in-drawn interest rate (LOANPRICE), and an indicator variable 1 if the loan is secured (LOANSEC). Borrower-level controls (BorrowerControls) include log of total assets (SIZE), total liabilities scaled by total assets (LEVERAGE), return on assets (ROA), market-to-book ratio $(M B)$, standard deviation of rolling five-year cash flow from operations scaled by beginning assets (STDCFO), standard deviation of rolling five-year sales scaled by beginning assets (STDSALE), proportion of losses over last five years (PROPLOSS), and log of operating cycle in days (OPCYCLE). To mitigate the concern of industry-specific omitted correlated variables, I include industry fixed effects. Year fixed effects are included to control for time-invariant macroeconomic factors.

\section{Data and Sample}

I primarily obtain data from two different sources - LPC Deascan database for loanlevel data and CRSP/Compustat merged dataset for firm-level data. To combine these datasets, I rely on Michael Roberts' link table that links Compustat with DealScan borrowers. ${ }^{16}$ My sample begins with loan facilities available in the LPC DealScan database between 1995 and 2013, for which I have access to the data. While Dealscan does not

\footnotetext{
${ }^{16}$ I thank Michael Roberts for the link tables.
} 
record all loans made, it provides information about most loans extended to public firms in the United States. While it covers around 50-75\% of all U.S. commercial loans in the early 1990s, the coverage increases substantially to around 90\% during 1992-2002 (Carey and Nini 2007). Dealscan provides loan-level information such as loan amount, maturity, interest rate, type, purpose, and covenants. Dealscan data are arranged at facility level and at package level. Loan facilities represent individual loans, while loan packages are a set of facilities issued by the common lead arranger with the similar set of covenants. Thus, my analyses are at the package-level.

Consistent with prior studies, I focus on the lead lender who plays an active role in originating the loan and monitoring the borrower, whereas participants are passive investors (Murfin 2012; Bushman, Hendricks and Williams 2016; Schwert 2018). I follow Ivashina (2009) to identify the lead lender(s). If a lender is reported as the "administrative agent," I define it as the lead lender. If no lender is reported as the "administrative agent," I define the lender who acts as the "agent," "arranger," "book-runner," "lead arranger," "lead bank," or "lead manager" as the lead lender. A lead lender identified at the facility level is also regarded as the lead lender at the package level. I then delete loan packages with more than one lead arranger because multi-lead lender loans make it difficult to isolate individual lender's incentive to influence borrowers' reporting decision.

I obtain firm financial data from Compustat-CRSP merged dataset for each of the fiscal year that is between the DealScan deal activation date and deal end date and that lags lenders financial data. Following prior studies, I exclude borrowers outside the USA and borrowers in the financial (SIC 6000-6999) and utility (SIC 4900-4999) industry. To avoid 
potential data error and outliers, I also exclude observations for which borrower's total asset is less than one million, common equity is negative, leverage is zero, or the market (book) leverage lies outside of the unit interval. Observations with missing values in regression model are also dropped from the sample. The final sample consists of 5,251 firm-year observations with 1,945 firms.

\section{RESULTS}

\section{Descriptive statistics}

Table 3.1 reports summary statistics for the variables used in regression estimations. During my sample period, the mean (median) strictness of debt covenant is 0.286 (0.077), which is mostly driven by strictness of performance covenants with mean (median) of $0.237(0.30)$. Although the sample only includes syndicated lending, there is a sizable variation in total assets of borrowing firms. The median bank in the sample has assets above $\$ 829$ million. Firms, on an average, use 27\% debt in the capital structure and earn $8.5 \%$ on their assets. During the sample, loans are made for an average of 45 months with 55\% loans being secured. Table 3.2 presents univariate correlation among the variables used in this study. As expected, the measures of financial reporting quality (AbsDACC and AbsPMDACC) are highly correlated (0.9156). Debt covenant strictness and the measures of covenants strictness are also positively correlated, indicating evidence in favor of debt covenant hypothesis. However, none of correlation coefficient creates multicollinearity problem in regression estimation as the variable inflation factors are all below 3.0. 


\section{Regression Estimation}

Panel A of Table 3.2 reports the baseline results from the fixed effect-based regression models. I report results in two separate columns for two different reporting quality measures. Columns (1) through (3) report the results for absolute value of discretionary accruals calculated from a modified Jones (1991) model, and columns (4) and (6) report results for performance-matched discretionary accruals. In all models, I include industry and year fixed effects. Each of models is highly significant $(\mathrm{p}<.001)$, and the explanatory power are consistent with prior studies. The sign and significance variables used in this study are also consistent with the results reported in prior studies. For example, firm size $(S I Z E)$ and profitability $(R O A)$ are negatively related to discretionary accruals, whereas cash flow volatility (STDCFO) and sales volatility (STDSALE) are positively associated.

In Model 1, the coefficient of PVIOL is positive but not significant, indicating that debt covenant strictness does not significantly affect the reporting quality of a firm. Consistently Model 2 reports similar findings. In table 3.4, I replace PVIOL by two variables, strictness of performance covenants $\left(P V I O L \_P\right)$ and strictness of capital covenants (PVIOL_C). Results show that for both the measures of financial reporting quality, the coefficients for $P V I O L \_P$ are negative but not statistically significant, while the coefficients for $P V I O L \_C$ are positive and significant. The difference in coefficients between $P V I O L \_P$ and $P V I O L \_C$ is also significant (p-value <.001). It supports the hypothesis that managers view performance covenants and capital covenants differently. The significantly positive coefficients for $P V I O L \_C$ also indicate that stricter capital covenants induce management to use higher discretion in earnings and thus reduce earnings 
quality. This is consistent with the hypothesis and prior studies (e.g. Demerjian 2011; Christensen et al. 2018) that capital covenants establish less monitoring on borrowers than performance covenants. Overall, findings provide evidence that debt covenant strictness in general does not affect financial reporting quality of a firm. However, if separated into performance and capital covenants, strictness of later covenants reduces reporting quality by encouraging management to use greater discretion.

\section{Instrumental variable: lender-specific shock}

One caveat of studying the effect of covenant tightness on financial reporting quality (FRQ) is that both debt contract and reporting quality are simultaneously and endogenously determined: the level of FRQ affects loan terms and the loan terms subsequently affect the level of FRQ ${ }^{17}$. I address this concern by using an instrumental variable (IV) based on the findings of Murfin (2012), who identifies lender-specific financial shock as a supply side determinant of debt covenants. The underlying assumption of using lender-specific shock as an IV is that the change in loan contract strictness is driven by shocks that affect only the lead arrangers and is plausibly exogenous to borrower characteristics. To obtain IV estimates, I regress the following first stage regression:

$$
\text { Strictness }_{i, t}=\alpha_{j}+\alpha_{t}+X_{i, t}^{\prime} b+\gamma \text { Defaults }{ }_{i, t-}+\epsilon_{i, t}
$$

In the second stage regression, I use the variation in covenant strictness calculated in the first stage and estimate the effect of covenant strictness on firm reporting quality:

\footnotetext{
${ }^{17}$ Like Spyridopoulos (2016), I argue that in the presence of simultaneity, the OLS estimates of covenant strictness on financial reporting quality are biased downwards. Using a system of equation, Spyridopoulos (2016) show that the bias introduced by simultaneous relationships, such as covenant strictness and firm operating efficiency, is negative.
} 


$$
F R Q_{i, t+1}=\alpha_{j}+\alpha_{t}+X^{\prime}{ }_{i, t} b+\delta \operatorname{Strictn} e s s_{l, t}+\eta_{i, t}
$$

The instrument that I use for covenant strictness is an exogenous shock to lender that is independent of borrower's fundamentals. Following Murfin (2012), I count the number of defaults that lead arrangers have suffered to their loan portfolios in the sixmonth period before issuing a new loan. However, to avoid the concern of capturing geography- or industry-specific economic conditions of the borrower, I follow Christensen et al (2018) and Spyridopoulos (2016) and exclude defaults in borrower's state and twodigit SIC industry. Consistent with Spyridopoulos (2016), mean default for a lender in the six-month period before issuing a new loan is 3. I rerun baseline regressions and report results for an instrumental variable in table 3.5 and table 3.6. Consistent with results reporting in table 3.3 and table 3.4 , second stage regression results indicate that the effect of instrumented debt covenant strictness on firm reporting quality is not statistically significant. However, if separated out, results indicate that strictness in capital covenants establish less monitoring on the management and thus reduce reporting quality. Overall, results using instrumental variable broadly confirm my main findings.

\section{CONCLUSION}

There has always been considerable interest in what determines the demand for, and supply of, financial reporting quality of a firm. A dominant force that emerges in the literature is the role of investors and lenders. Ball et al. (2008) argue that the demand for financial reporting primarily emerges from the debt market than equity market. In this paper, I document an important supply-side determinant of borrowers' reporting quality. Using a comprehensive measure of debt covenant strictness, I document that stricter 
covenants do not necessarily establish better monitoring and therefore do not change reporting quality of a firm. I also show that strictness in capital covenants, as oppose to that in performance covenants, encourage management to use greater discretion, which leads to a reduction in reporting quality. As I document in this paper, the covenant channel in affecting the reporting quality of a firm extends the large amount of literature that examines the role of lenders in affecting reporting decision of a firm (Ahmed et al. 2002; Ball and Shivakumar 2005; Beatty et al. 2008; Wittenberg-Moerman 2008; Zhang 2008; Armstrong, Guay, and Weber 2010). Overall, this study extends our understanding on how lenders establish monitoring mechanisms that mitigate agency conflicts among various stakeholders.

\section{REFERENCES}

Armstrong, C.S., Guay, W.R. and Weber, J.P., 2010. The role of information and financial reporting in corporate governance and debt contracting. Journal of Accounting and Economics, 50(2-3), pp.179-234.

Ball, R., Robin, A. and Sadka, G., 2008. Is financial reporting shaped by equity markets or by debt markets? An international study of timeliness and conservatism. Review of accounting studies, 13(2-3), pp.168-205.

Basu, S., 1997. The conservatism principle and the asymmetric timeliness of earnings1. Journal of accounting and economics, 24(1), pp.3-37.

Beatty, A. and Weber, J., 2003. The effects of debt contracting on voluntary accounting method changes. The Accounting Review, 78(1), pp.119-142.

Beneish, M.D. and Press, E., 1993. Costs of technical violation of accounting-based debt covenants. Accounting Review, pp.233-257.

Beneish, M.D., 1999. Incentives and penalties related to earnings overstatements that violate GAAP. The Accounting Review, 74(4), pp.425-457. 
Bharath, S.T., Sunder, J. and Sunder, S.V., 2008. Accounting quality and debt contracting. The Accounting Review, 83(1), pp.1-28.

Bhaskar, L.S., Krishnan, G.V. and Yu, W., 2017. Debt covenant violations, firm financial distress, and auditor actions. Contemporary Accounting Research, 34(1), pp.186-215.

Billett, M.T., KING, T.H.D. and Mauer, D.C., 2007. Growth opportunities and the choice of leverage, debt maturity, and covenants. The Journal of Finance, 62(2), pp.697730 .

Bradley, Michael and Roberts, Michael R., The Structure and Pricing of Corporate Debt Covenants (March 11, 2004). 6th Annual Texas Finance Festival. Available at SSRN: https://ssrn.com/abstract=585882 or http://dx.doi.org/10.2139/ssrn.585882

Burgstahler, D.C., Hail, L. and Leuz, C., 2006. The importance of reporting incentives: Earnings management in European private and public firms. The accounting review, 81(5), pp.983-1016.

Bushman, R.M. and Piotroski, J.D., 2006. Financial reporting incentives for conservative accounting: The influence of legal and political institutions. Journal of Accounting and Economics, 42(1-2), pp.107-148.

Chava, S. and Roberts, M.R., 2008. How does financing impact investment? The role of debt covenants. The Journal of Finance, 63(5), pp.2085-2121.

Christensen, H.B. and Nikolaev, V.V., 2012. Capital versus performance covenants in debt contracts. Journal of Accounting Research, 50(1), pp.75-116.

Christensen, H.B., Nikolaev, V.V. and WITTENBERG-MOERMAN, R.E.G.I.N.A., 2016. Accounting information in financial contracting: The incomplete contract theory perspective. Journal of accounting research, 54(2), pp.397-435.

Christensen, Hans Bonde and Macciocchi, Daniele and Nikolaev, Valeri V., Financial Shocks and Corporate Investment Activity: The Role of Financial Covenants (March 1, 2018). Available at SSRN: https://ssrn.com/abstract $=3079996$ or http://dx.doi.org/10.2139/ssrn.3079996

DeAngelo, H., DeAngelo, L. and Skinner, D.J., 1994. Accounting choice in troubled companies. Journal of accounting and economics, 17(1-2), pp.113-143.

DeFond, M.L. and Jiambalvo, J., 1994. Debt covenant violation and manipulation of accruals. Journal of accounting and economics, 17(1-2), pp.145-176.

Demeriian, P.R. and Owens, E.L., 2016. Measuring the probability of financial covenant violation in private debt contracts. Journal of Accounting and Economics, 61(2-3), pp.433-447. 
Demerjian, P.R., 2011. Accounting standards and debt covenants: Has the "balance sheet approach" led to a decline in the use of balance sheet covenants? Journal of Accounting and Economics, 52(2-3), pp.178-202.

Demiroglu, C. and James, C.M., 2010. The information content of bank loan covenants. The Review of Financial Studies, 23(10), pp.3700-3737.

Dichev, I.D. and Skinner, D.J., 2002. Large-sample evidence on the debt covenant hypothesis. Journal of accounting research, 40(4), pp.1091-1123.

Erkens, D.H., Subramanyam, K.R. and Zhang, J., 2014. Affiliated banker on board and conservative accounting. The Accounting Review, 89(5), pp.1703-1728.

Ertan, A. and Karolyi, S.A., 2014. Debt covenants, expected default costs, and the implicit cost of financial reporting manipulation.

Fang, V.W., Huang, A.H. and Karpoff, J.M., 2016. Short selling and earnings management: A controlled experiment. The Journal of Finance, 71(3), pp.1251-1294.

Fields, T.D., Lys, T.Z. and Vincent, L., 2001. Empirical research on accounting choice. Journal of accounting and economics, 31(1-3), pp.255-307.

Frankel, Richard M. and Litov, Lubomir P., Financial Accounting Characteristics and Debt Covenants (April 2007). Available at SSRN: https://ssrn.com/abstract=978711 or http://dx.doi.org/10.2139/ssrn.978711

Franz, D.R., HassabElnaby, H.R. and Lobo, G.J., 2014. Impact of proximity to debt covenant violation on earnings management. Review of Accounting Studies, 19(1), pp.473-505.

Gao, Y., Khan, M. and Tan, L., 2017. Further evidence on consequences of debt covenant violations. Contemporary accounting research, 34(3), pp.1489-1521.

Gigler, F., Kanodia, C., Sapra, H. and Venugopalan, R., 2009. Accounting conservatism and the efficiency of debt contracts. Journal of accounting research, 47(3), pp.767797.

Givoly, D., Hayn, C. and Katz, S., 2017. The changing relevance of accounting information to debt holders over time. Review of Accounting Studies, 22(1), pp.64-108.

Graham, J.R., Harvey, C.R. and Rajgopal, S., 2005. The economic implications of corporate financial reporting. Journal of accounting and economics, 40(1-3), pp.373.

Green, D., 2017. Corporate Refinancing, Covenants, and the Agency Cost of Debt. Working paper, MIT Sloan School of Management. 
Gul, F.A. and Goodwin, J., 2010. Short-term debt maturity structures, credit ratings, and the pricing of audit services. The Accounting Review, 85(3), pp.877-909.

Harris, M. and Raviv, A., 1991. The theory of capital structure. the Journal of Finance, 46(1), pp.297-355.

Healy, P.M. and Palepu, K.G., 1990. Effectiveness of accounting-based dividend covenants. Journal of accounting and Economics, 12(1-3), pp.97-123.

Jensen, M.C. and Meckling, W.H., 1976. Theory of the firm: Managerial behavior, agency costs and ownership structure. Journal of financial economics, 3(4), pp.305-360.

Khan, M. and Watts, R.L., 2009. Estimation and empirical properties of a firm-year measure of accounting conservatism. Journal of accounting and Economics, 48(2-3), pp.132-150.

Khan, Urooj and Lo, Alvis K., Bank Lending Standards and Borrower Accounting Conservatism (March 9, 2018). Columbia Business School Research Paper No. 1795. Available at SSRN: https://ssrn.com/abstract=3037739 or http://dx.doi.org/10.2139/ssrn.3037739

Kothari, S.P., Leone, A.J. and Wasley, C.E., 2005. Performance matched discretionary accrual measures. Journal of accounting and economics, 39(1), pp.163-197.

Leuz, C., Nanda, D. and Wysocki, P.D., 2003. Earnings management and investor protection: an international comparison. Journal of financial economics, 69(3), pp.505-527.

Li, Xi and Tuna, A. Irem and Vasvari, Florin P., Corporate Governance and Covenants in Debt Contracts (April 30, 2014). Available at SSRN: https://ssrn.com/abstract=1988272 or http://dx.doi.org/10.2139/ssrn.1988272

McNichols, M.F. and Stubben, S.R., 2008. Does earnings management affect firms' investment decisions?. The accounting review, 83(6), pp.1571-1603.

Murfin, J., 2012. The supply-side determinants of loan contract strictness. The Journal of Finance, 67(5), pp.1565-1601.

Myers, S.C., 1977. Determinants of corporate borrowing. Journal of financial economics, 5(2), pp.147-175.

Nikolaev, V.V., 2010. Debt covenants and accounting conservatism. Journal of Accounting Research, 48(1), pp.51-89.

Nini, G., Smith, D.C. and Sufi, A., 2012. Creditor control rights, corporate governance, and firm value. The Review of Financial Studies, 25(6), pp.1713-1761. 
Prilmeier, R., 2017. Why do loans contain covenants? Evidence from lending relationships. Journal of Financial Economics, 123(3), pp.558-579.

Roberts, M.R. and Sufi, A., 2009. Renegotiation of financial contracts: Evidence from private credit agreements. Journal of Financial Economics, 93(2), pp.159-184.

Smith Jr, C.W. and Warner, J.B., 1979. On financial contracting: An analysis of bond covenants. Journal of financial economics, 7(2), pp.117-161.

Spyridopoulos, Ioannis, Tough Love: The Causal Effects of Debt Contract Design on Firm Performance (May 19, 2018). Available at SSRN: https://ssrn.com/abstract=2551333 or http://dx.doi.org/10.2139/ssrn.2551333

Stubben, S.R., 2010. Discretionary revenues as a measure of earnings management. The accounting review, 85(2), pp.695-717.

Sunder, J., Sunder, S.V. and Zhang, J., 2018. Balance sheet conservatism and debt contracting. Contemporary Accounting Research, 35(1), pp.494-524.

Sweeney, A.P., 1994. Debt-covenant violations and managers' accounting responses. Journal of accounting and Economics, 17(3), pp.281-308.

Tan, L., 2013. Creditor control rights, state of nature verification, and financial reporting conservatism. Journal of Accounting and Economics, 55(1), pp.1-22.

Teoh, S.H., Welch, I. and Wong, T.J., 1998. Earnings management and the long-run market performance of initial public offerings. The Journal of Finance, 53(6), pp.1935-1974.

Vashishtha, R., 2014. The role of bank monitoring in borrowers' discretionary disclosure: Evidence from covenant violations. Journal of Accounting and Economics, 57(2-3), pp.176-195.

Wang, J., 2017. Debt covenant design and creditor control rights: Evidence from the tightest covenant. Journal of Corporate Finance, 44, pp.331-352.

Watts, R. and Zimmerman, J., 1986. J.,(1986): Positive accounting theory. prentice hall.

Zhang, J., 2008. Efficiency gains from accounting conservatism: Benefits to lenders and borrowers. Journal of Accounting and Economics, 45(1), pp.27-54. 
TABLE 3.1

Descriptive Statistics

\begin{tabular}{lcccccc}
\hline Variables & Observations & Mean & S.D. & $1^{\text {st }}$ quartile & Median & $3^{\text {rd }}$ quartile \\
\hline PVIOL & 5,251 & 0.286 & 0.369 & 0.010 & 0.077 & 0.550 \\
PVIOL_P & 5,251 & 0.237 & 0.356 & 0.000 & 0.030 & 0.330 \\
PVIOL_C & 5,251 & 0.072 & 0.193 & 0.000 & 0.000 & 0.042 \\
NUMDEF & 5,251 & 2.998 & 3.604 & 0.000 & 1.000 & 5.000 \\
AbsDACC & 5,251 & 0.059 & 0.064 & 0.017 & 0.039 & 0.076 \\
AbsPMDACC & 5,251 & 0.055 & 0.058 & 0.016 & 0.037 & 0.070 \\
SIZE & 5,251 & 20.537 & 1.741 & 19.326 & 20.536 & 21.761 \\
LEVERAGE & 5,251 & 0.275 & 0.162 & 0.155 & 0.267 & 0.383 \\
ROA & 5,251 & 0.085 & 0.079 & 0.051 & 0.088 & 0.126 \\
MB & 5,251 & 2.806 & 2.705 & 1.325 & 2.069 & 3.275 \\
STDCFO & 5,251 & 0.058 & 0.051 & 0.025 & 0.043 & 0.073 \\
STDSALE & 5,251 & 0.262 & 0.279 & 0.090 & 0.169 & 0.315 \\
PROPLOSS & 5,251 & 0.725 & 1.036 & 0.000 & 0.000 & 1.000 \\
OPCYLCE & 5,251 & 4.696 & 0.658 & 4.393 & 4.771 & 5.107 \\
LOANAMT & 5,251 & 18.898 & 1.564 & 17.910 & 19.114 & 20.030 \\
LOANMAT & 5,251 & 3.687 & 0.555 & 3.555 & 3.871 & 4.094 \\
LOANPRICE & 5,251 & 4.875 & 0.758 & 4.354 & 5.011 & 5.416 \\
LOANSEC & 5,251 & 0.552 & 0.497 & 0.000 & 1.000 & 1.000 \\
& & & & & & \\
\hline
\end{tabular}


TABLE 3.2

Correlation Matrix

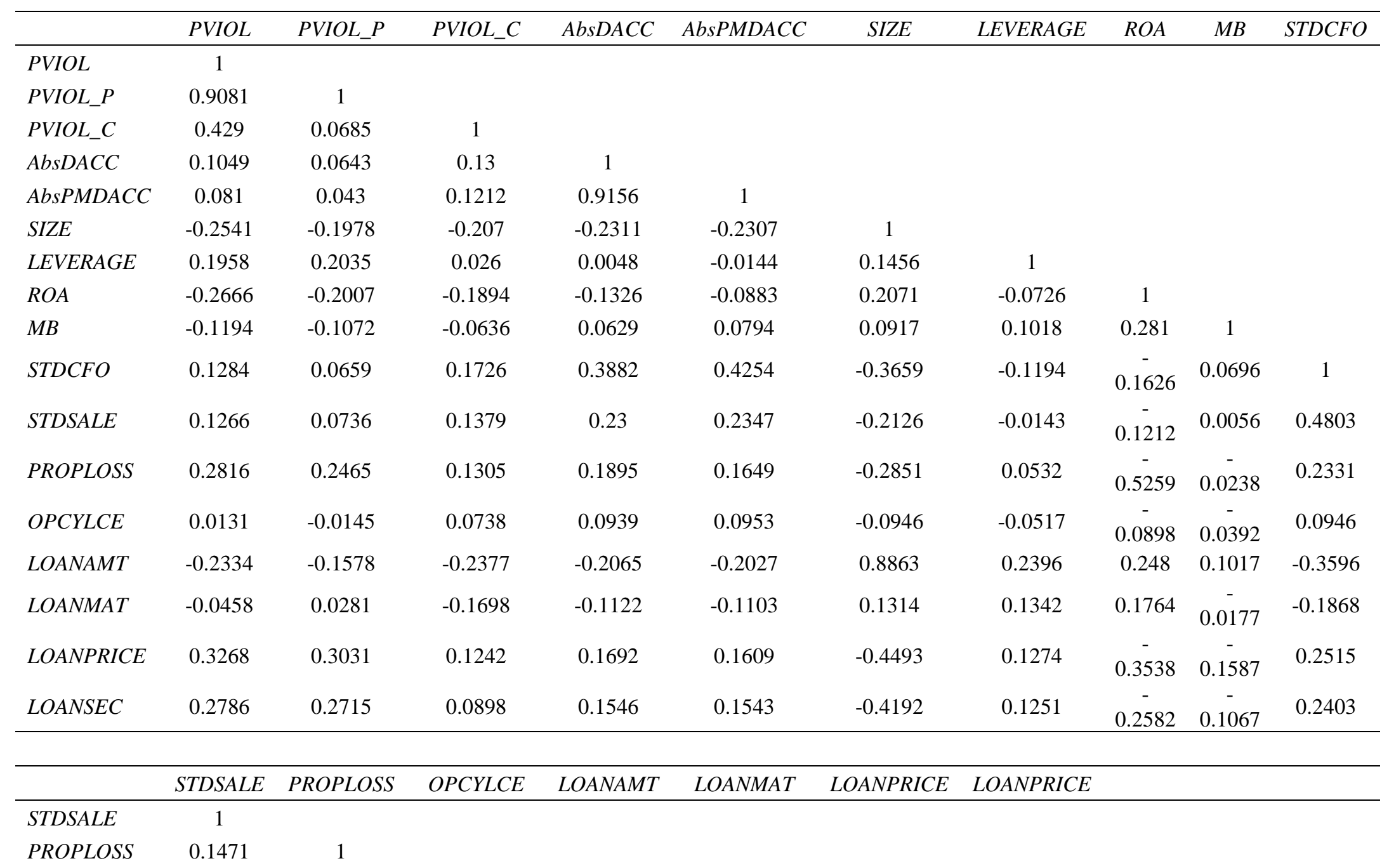




\begin{tabular}{lccccccc} 
OPCYLCE & -0.1425 & 0.0435 & 1 & & & & \\
LOANAMT & -0.1963 & -0.3169 & -0.1031 & 1 & & & \\
LOANMAT & -0.1043 & -0.1295 & -0.0969 & 0.3017 & 1 & & \\
LOANPRICE & 0.1824 & 0.4182 & 0.0272 & -0.3975 & -0.0149 & 1 & 1 \\
LOANSEC & 0.1783 & 0.3472 & 0.0057 & -0.3291 & 0.071 & 0.5962 & 1 \\
\hline
\end{tabular}


TABLE 3.3

Regression Analyses of Financial Reporting Quality on Debt Covenant Strictness

\begin{tabular}{|c|c|c|c|c|c|c|}
\hline & \multicolumn{3}{|c|}{ Dep Var: AbsDACC } & \multicolumn{3}{|c|}{ Dep Var: AbsPMDACC } \\
\hline VARIABLES & Coefficient & $t$-stat & p-value & Coefficients & $t$-stat & p-value \\
\hline PVIOL & 0.001 & 0.232 & 0.817 & -0.002 & -0.659 & 0.510 \\
\hline SIZE & $-0.003 * * *$ & -2.684 & 0.007 & $-0.003 * * *$ & -2.724 & 0.007 \\
\hline LEVERAGE & 0.007 & 0.883 & 0.377 & 0.002 & 0.248 & 0.804 \\
\hline$R O A$ & -0.007 & -0.336 & 0.737 & 0.019 & 1.078 & 0.281 \\
\hline$M B$ & $0.001 * * *$ & 2.985 & 0.003 & $0.001 * * *$ & 3.245 & 0.001 \\
\hline STDCFO & $0.339 * * *$ & 10.189 & 0.000 & $0.369 * * *$ & 12.690 & 0.000 \\
\hline STDSALE & $0.012 * *$ & 2.362 & 0.018 & 0.007 & 1.565 & 0.118 \\
\hline PROPLOSS & $0.004 * * *$ & 3.382 & 0.001 & $0.003 * * *$ & 2.979 & 0.003 \\
\hline OPCYLCE & $0.006 * * *$ & 2.936 & 0.003 & $0.004 *$ & 1.808 & 0.071 \\
\hline LOANAMT & $0.004 * * *$ & 2.697 & 0.007 & $0.004 * * *$ & 3.042 & 0.002 \\
\hline LOANMAT & $-0.004 *$ & -1.931 & 0.054 & $-0.003 * *$ & -2.002 & 0.045 \\
\hline LOANPRICE & $0.005 * * *$ & 2.601 & 0.009 & $0.005 * * *$ & 2.820 & 0.005 \\
\hline LOANSEC & 0.000 & 0.069 & 0.945 & 0.002 & 0.789 & 0.430 \\
\hline Constat & -0.046 & -1.497 & 0.135 & -0.040 & -1.501 & 0.134 \\
\hline Year FE & Yes & & & Yes & & \\
\hline Industry FE & Yes & & & Yes & & \\
\hline Observations & 5,251 & & & 5,251 & & \\
\hline R-squared & 0.225 & & & 0.234 & & \\
\hline
\end{tabular}

Sample includes firm-year observations with available data in both LPC dealscan database and in CRSP/Compustat merged dataset 1995 to 2013. Primary variable of interest, PVIOL, defined as the probability of covenant violation, measures debt covenant strictness. All other variables in the table are defined in Appendix I. Industry dummies capture industry fixed effects with two-digit SIC code classifications. Robust standard errors are clustered by firm (gvkey). All continuous variables are winsorized at 1 percent and 99 percent. $* * *, * *$, and $*$ respectively denote significance levels of $1 \%, 5 \%$, and $10 \%$. 
TABLE 3.4

Regression Analyses of Financial Reporting Quality on Debt Covenant Strictness

\begin{tabular}{|c|c|c|c|c|c|c|}
\hline \multirow[b]{2}{*}{ VARIABLES } & \multicolumn{3}{|c|}{ Dep Var: AbsDACC } & \multicolumn{3}{|c|}{ Dep Var: AbsPMDACC } \\
\hline & Coefficient & $t$-stat & $p$-value & Coefficients & $t$-stat & p-value \\
\hline PVIOL_P & -0.001 & -0.433 & 0.665 & -0.003 & -1.100 & 0.272 \\
\hline PVIOL_C & $0.012 * *$ & 2.071 & 0.038 & $0.009 *$ & 1.806 & 0.071 \\
\hline SIZE & $-0.003 * * *$ & -2.743 & 0.006 & $-0.003 * * *$ & -2.777 & 0.006 \\
\hline LEVERAGE & 0.007 & 0.904 & 0.366 & 0.002 & 0.252 & 0.801 \\
\hline$R O A$ & -0.004 & -0.208 & 0.835 & 0.022 & 1.219 & 0.223 \\
\hline$M B$ & $0.001 * * *$ & 2.971 & 0.003 & $0.001 * * *$ & 3.232 & 0.001 \\
\hline STDCFO & $0.336 * * *$ & 10.122 & 0.000 & $0.367 * * *$ & 12.642 & 0.000 \\
\hline STDSALE & $0.011 * *$ & 2.279 & 0.023 & 0.007 & 1.472 & 0.141 \\
\hline PROPLOSS & $0.004 * * *$ & 3.467 & 0.001 & $0.004 * * *$ & 3.046 & 0.002 \\
\hline OPCYLCE & $0.006 * * *$ & 2.886 & 0.004 & $0.003 *$ & 1.759 & 0.079 \\
\hline LOANAMT & $0.004 * * *$ & 2.883 & 0.004 & $0.004 * * *$ & 3.225 & 0.001 \\
\hline LOANMAT & $-0.003^{*}$ & -1.724 & 0.085 & $-0.003^{*}$ & -1.807 & 0.071 \\
\hline LOANPRICE & $0.005 * * *$ & 2.610 & 0.009 & $0.005 * * *$ & 2.813 & 0.005 \\
\hline LOANSEC & 0.000 & 0.137 & 0.891 & 0.002 & 0.853 & 0.394 \\
\hline Constat & -0.050 & -1.642 & 0.101 & $-0.044^{*}$ & -1.647 & 0.100 \\
\hline Year FE & Yes & & & Yes & & \\
\hline Industry FE & Yes & & & Yes & & \\
\hline Observations & 5,251 & & & 5,251 & & \\
\hline R-squared & 0.226 & & & 0.235 & & \\
\hline
\end{tabular}

Sample includes firm-year observations with available data in both LPC dealscan database and in CRSP/Compustat merged dataset 1995 to 2013. Primary variables of interest, $P V I O L \_P$ and PVIOL_C , defined as the probability of covenant violation, respectively measure strictness of performance covenants and strictness of capital covenants. All other variables in the table are defined in Appendix I. Industry dummies capture industry fixed effects with two-digit SIC code classifications. Robust standard errors are clustered by firm (gvkey). All continuous variables are winsorized at 1 percent and 99 percent. ***,**, and * respectively denote significance levels of $1 \%, 5 \%$, and $10 \%$. 
TABLE 3.5

Regression Analyses of Financial Reporting Quality on Debt Covenant Strictness (Using Instrumental Variable)

\begin{tabular}{|c|c|c|c|c|c|c|}
\hline & \multicolumn{3}{|c|}{ Dep Var: AbsDACC } & \multicolumn{3}{|c|}{ Dep Var: AbsPMDACC } \\
\hline VARIABLES & Coefficient & $t$-stat & $p$-value & Coefficients & $t$-stat & $p$-value \\
\hline$\widehat{P V I O L}$ & 0.001 & 0.271 & 0.786 & -0.002 & -0.765 & 0.444 \\
\hline SIZE & $-0.003 * * *$ & -2.991 & 0.003 & $-0.003 * * *$ & -2.980 & 0.003 \\
\hline LEVERAGE & 0.007 & 1.125 & 0.261 & 0.002 & 0.317 & 0.751 \\
\hline$R O A$ & -0.007 & -0.560 & 0.575 & $0.019 *$ & 1.689 & 0.091 \\
\hline$M B$ & $0.001 * * *$ & 4.441 & 0.000 & $0.001 * * *$ & 4.680 & 0.000 \\
\hline STDCFO & $0.339 * * *$ & 17.154 & 0.000 & $0.369 * * *$ & 20.887 & 0.000 \\
\hline STDSALE & $0.012 * * *$ & 3.357 & 0.001 & $0.007 * *$ & 2.214 & 0.027 \\
\hline PROPLOSS & $0.004 * * *$ & 4.417 & 0.000 & $0.003 * * *$ & 3.948 & 0.000 \\
\hline OPCYLCE & $0.006 * * *$ & 3.547 & 0.000 & $0.004 * *$ & 2.180 & 0.029 \\
\hline LOANAMT & $0.004 * * *$ & 2.906 & 0.004 & $0.004 * * *$ & 3.270 & 0.001 \\
\hline LOANMAT & $-0.004 * *$ & -2.138 & 0.033 & $-0.003 * *$ & -2.175 & 0.030 \\
\hline LOANPRICE & $0.005 * * *$ & 2.988 & 0.003 & $0.005 * * *$ & 3.185 & 0.001 \\
\hline LOANSEC & 0.000 & 0.074 & 0.941 & 0.002 & 0.811 & 0.417 \\
\hline Constat & -0.046 & -1.451 & 0.147 & -0.040 & -1.423 & 0.155 \\
\hline Year FE & Yes & & & Yes & & \\
\hline Industry FE & Yes & & & Yes & & \\
\hline Observations & 5,251 & & & 5,251 & & \\
\hline R-squared & 0.225 & & & 0.234 & & \\
\hline
\end{tabular}

Sample includes firm-year observations with available data in both LPC dealscan database and in CRSP/Compustat merged dataset 1995 to 2013. Primary variable of interest, PVIOL, defined as the probability of covenant violation, measures debt covenant strictness. All other variables in the table are defined in Appendix I. Industry dummies capture industry fixed effects with two-digit SIC code classifications. Robust standard errors are clustered by firm (gvkey). All continuous variables are winsorized at 1 percent and 99 percent. $* * *, * *$, and $*$ respectively denote significance levels of $1 \%, 5 \%$, and $10 \%$. 
TABLE 3.6

\section{Regression Analyses of Financial Reporting Quality on Debt Covenant Strictness (Using Instrumental Variable)}

\begin{tabular}{|c|c|c|c|c|c|c|}
\hline & \multicolumn{3}{|c|}{ Dep Var: AbsDACC } & \multicolumn{3}{|c|}{ Dep Var: AbsPMDACC } \\
\hline VARIABLES & Coefficient & $t$-stat & $p$-value & Coefficients & $t$-stat & $p$-value \\
\hline$P \widehat{V I O L} \_P$ & -0.001 & -0.518 & 0.605 & -0.003 & -1.321 & 0.187 \\
\hline$P V \widehat{V I O L}_{-} C$ & $0.012 * * *$ & 2.748 & 0.006 & $0.009 * *$ & 2.299 & 0.022 \\
\hline$S I Z E$ & $-0.003 * * *$ & -3.045 & 0.002 & $-0.003 * * *$ & -3.035 & 0.002 \\
\hline LEVERAGE & 0.007 & 1.155 & 0.248 & 0.002 & 0.323 & 0.747 \\
\hline$R O A$ & -0.004 & -0.348 & 0.728 & $0.022 *$ & 1.912 & 0.056 \\
\hline$M B$ & $0.001 * * *$ & 4.425 & 0.000 & $0.001 * * *$ & 4.668 & 0.000 \\
\hline STDCFO & $0.336 * * *$ & 17.027 & 0.000 & $0.367 * * *$ & 20.762 & 0.000 \\
\hline STDSALE & $0.011 * * *$ & 3.222 & 0.001 & $0.007 * *$ & 2.074 & 0.038 \\
\hline PROPLOSS & $0.004 * * *$ & 4.534 & 0.000 & $0.004 * * *$ & 4.043 & 0.000 \\
\hline OPCYLCE & $0.006 * * *$ & 3.494 & 0.000 & $0.003 * *$ & 2.123 & 0.034 \\
\hline LOANAMT & $0.004 * * *$ & 3.085 & 0.002 & $0.004 * * *$ & 3.449 & 0.001 \\
\hline$L O A N M A T$ & $-0.003^{*}$ & -1.914 & 0.056 & $-0.003^{*}$ & -1.959 & 0.050 \\
\hline LOANPRICE & $0.005 * * *$ & 3.002 & 0.003 & $0.005 * * *$ & 3.181 & 0.001 \\
\hline LOANSEC & 0.000 & 0.145 & 0.884 & 0.002 & 0.879 & 0.380 \\
\hline Constat & -0.050 & -1.593 & 0.111 & -0.044 & -1.558 & 0.119 \\
\hline Year FE & Yes & & & Yes & & \\
\hline Industry FE & Yes & & & Yes & & \\
\hline Observations & 5,251 & & & 5,251 & & \\
\hline R-squared & 0.226 & & & 0.235 & & \\
\hline
\end{tabular}

Sample includes firm-year observations with available data in both LPC dealscan database and in CRSP/Compustat merged dataset 1995 to 2013. Primary variables of interest, PVIOL_P and $P V I O L \_C$, defined as the probability of covenant violation, respectively measure strictness of performance covenants and strictness of capital covenants. All other variables in the table are defined in Appendix I. Industry dummies capture industry fixed effects with two-digit SIC code classifications. Robust standard errors are clustered by firm (gvkey). All continuous variables are winsorized at 1 percent and 99 percent. ***,**, and * respectively denote significance levels of $1 \%, 5 \%$, and $10 \%$. 


\section{APPENDIX C}

Variable Definition:

PVIOL: Probability of Covenant Violation, following Demerjian et al. (2016)

PVIOL_P: Probability of Performance Covenant Violation, following Demerjian et al. (2016)

PVIOL_C: Probability of Capital Covenant Violation, following Demerjian et al. (2016)

NUMDEF: Number of defaults in the portfolio of the lead lender in the six-month period prior to the origination of the loan

AbsDACC: Absolute value difference between total accruals and expected accruals, calculated from the modified version of Jones (1991)

AbsPMDACC: The difference between abnormal accrual and median industry-ROA decile abnormal accrual, as suggested by Kothari et al. (2005)

SIZE: Natural logarithm of total assets

LEVERAGE: Total debt scaled by total assets

ROA: Market-to-Book ratio

$M B$ : Earnings before interest and taxes scaled by lagged assets

STDCFO: Standard deviation of 5-year cash flow from operations scaled by lagged total assets

STDSALE: Standard deviation of 5-year sales scaled by lagged total assets

PROPLOSS: Proportion of losses over the last five years

OPCYLCE: Natural logarithm of accounts receivable turnover (in days) and inventory turnover (in days)

LOANAMT: Natural logarithm of dollar amount of loan

LOANMAT: Natural logarithm of maturity of the loan in months

LOANPRICE: Natural logarithm of all-in-drawn interest rate

LOANSEC: 1 if the loan is secured, 0 otherwise 
VITA

\section{SAFAYAT HOSSAIN}

Born, Feni, Bangladesh

2007

B.B.A., Accounting (Summa Cum Laude)

University of Dhaka

Dhaka, Bangladesh

2009

M.B.A., Accounting (Summa Cum Laude)

University of Dhaka

Dhaka, Bangladesh

2012

M.S., Finance

Clark University

Worcester, Massachusetts

2015-2019 Graduate Teaching Assistant

Florida International University

Miami, Florida

\section{PUBLICATIONS AND PRESENTATIONS}

Barua, A., Md Safayat Hossain and Dasaratha Rama. 2019. Financial vs Operating Liability Leverage and Audit Fees. International Journal of Auditing (Forthcoming) 